\title{
Derivation of the Functional Renormalization Group $\beta$-function at order $1 / N$ for manifolds pinned by disorder
}

\author{
Pierre Le Doussal ${ }^{1}$ and Kay Jörg Wiese ${ }^{1,2}$ \\ ${ }^{1}$ CNRS-Laboratoire de Physique Théorique de l'Ecole Normale Supérieure, 24 rue Lhomond, 75005 Paris, France \\ ${ }^{2}$ Institut für Theoretische Physik, Universität zu Köln, Zülpicher Str. 77, 50937 Köln, Germany
}

August 29, 2018

\begin{abstract}
In an earlier publication, we have introduced a method to obtain, at large $N$, the effective action for $d$-dimensional manifolds in a $N$-dimensional disordered environment. This allowed to obtain the Functional Renormalization Group (FRG) equation for $N=\infty$ and was shown to reproduce, with no need for ultrametric replica symmetry breaking, the predictions of the Mézard-Parisi solution. Here we compute the corrections at order $1 / N$. We introduce two novel complementary methods, a diagrammatic and an algebraic one, to perform the complicated resummation of an infinite number of loops, and derive the $\beta$-function of the theory to order $1 / N$. We present both the effective action and the corresponding functional renormalization group equations. The aim is to explain the conceptual basis and give a detailed account of the novel aspects of such calculations. The analysis of the FRG flow, comparison with other studies, and applications, e.g. to the strong-coupling phase of the Kardar-Parisi-Zhang equation are examined in a subsequent publication.
\end{abstract}

\section{Introduction}

In a series of recent articles we have constructed the Functional Renormalization Group (FRG) method for disordered systems, applied to specific situations and beyond one loop [1-9]. This method is, apart from mean field theory [11,12] using Replica Symmetry Breaking (RSB) and some rare exactly solvable cases $[13,14]$, the only known analytical method which promises to handle the strong coupling glass phase of disordered elastic systems [1-9,15-38]. Such systems, modeled by an elastic manifold (of internal dimension $d$ ) with a $N$-component displacement field $u(x)$ (i.e. $x \in \mathbb{R}^{d}$ and $u(x) \in \mathbb{R}^{N}$ ), are of high interest for numerous experiments [39-43,25,36,44]. This so-called random manifold model still offers great theoretical challenges and a strong motivation is the hope to gain insight into glassy physics. In addition, the $d=1$ case maps onto the much studied Kardar-Parisi-Zhang growth equation [45]. It exhibits a strong coupling phase for which the upper critical dimension is still under debate [46-50].

Higher loop studies of the statics of disordered elastic systems allow, in principle, a systematic dimensional expansion, in the simplest case around $d=4$. They are however of a rather different nature than in standard field theory for pure critical systems [1,7,10,31,33-35,51-54]. Thermal fluctuations are found to be formally irrelevant in these glass phases, suggesting that the physics is controlled by a zero temperature fixed point. However before this fixed point is reached, the zero temperature effective action is found to become non-analytic [17]. Although this allows to evade the so-called dimensional reduction [55] which makes naive perturbation theory useless and yields unphysical results, it also generates amazing new subtleties in the field theory. These were analyzed in a number of papers [1,7,10,31,33-35,51-54], and although some solutions to the puzzles were proposed the physics still remains to be elucidated. 
An interesting limit where one can hope to gain insight into these formidable problems is the large$N$ limit. Since $N=\infty$ is formally the mean-field limit, it allows a direct confrontation between the FRG method and mean field methods. A solution of the $O(N)$ random manifold model for $N=\infty$ was proposed by Mézard and Parisi, using a saddle point with spontaneous replica symmetry breaking $[11,12]$. As in other models of glasses, spontaneous RSB can be related to ergodicity breaking of the Gibbs measure into several ground states [56]. Although it offers a rather elegant way out of dimensional reduction, it is by no means clear that systems with (large but) finite $N$ should exhibit such a tremendous degeneracy of low energy states; and there are in fact indications to the contrary [57].

It is thus crucial to develop another line of attack, even in that limit. This is what we have achieved in a previous publication, where we have computed the effective action of the theory at large $N$. There we have derived the $\beta$-function of the field theory to dominant order, i.e. for $N=\infty[2,3,8]$. We have discovered that beyond the Larkin length the FRG flow freezes (at least for specific initial conditions) and that most of the features of the Mézard Parisi solution can be recovered. Interestingly however, in this formulation there is no need for a spontaneous RSB ansatz with ultrametric structure. Thus one may hope that it could be more adapted to real world situations than the RSB calculations. Such RSB calculations of fluctuations around the mean-field solution have been attempted for the random-manifold problem only in the case of 1-step non-marginal RSB (with disappointing result [58]) and offer, in full generality, extreme complications, as is illustrated by several studies for spin glasses [59-66].

The next challenge is thus to extend the FRG in a large- $N$ expansion beyond the dominant order $(N=$ $\infty)$. This is the aim of the present paper. Since this is a complicated calculation, and involves developing new methods which are of interest by themselves, this paper is restricted to the calculation of the effective action and derivation of the $\beta$-function to order $1 / N$. This is performed at $T=0$ and at finite temperature. The analysis of the resulting FRG flow, comparison with other studies, and applications, e.g. to the strongcoupling phase of the KPZ equation is involved and is the subject of a forthcoming publication.

The outline of the paper is as follows. In section 2 we give the general formulation of the $1 / N$ expansion for the effective action of the random manifold. Details and generalizations are given in appendices A, B, and $\mathrm{C}$. In section 3 we summarize the main results for $N=\infty$. Section 4 explains the derivation of the $1 / N$ correction by a graphical method, which introduces a new type of diagrammatics. Section 5 explains the principle of a second and complementary method based on the algebra of 4-replica tensors. Section 6 contains the full result for the effective action to order $1 / N$, first expressed in bare parameters, then as a function of the renormalized dimensionless disorder. This allows, in section 7 , for a derivation of the $\beta$-function at $T=0$. The structure of the finite- $T \beta$-function is indicated, and details given in appendix $\mathrm{H}$. A fool-proof diagrammatic version for finite temperature is given in appendix D. More details on the two main methods are given respectively in appendix $\mathrm{G}$ (for the diagrammatic method, including an alternative derivation of the $T=0 \beta$-function) and in appendix $\mathrm{F}$ (for the algebraic method). Appendix I contains a list of all integrals. A table summarizing the notation is found in appendix $\mathrm{J}$.

\section{$21 / N$ expansion of the effective action: General formula}

We start from the partition function of an interface $\mathcal{Z}_{V}=\int \mathcal{D}[u] \mathrm{e}^{-\mathcal{H}_{V}[u] / T}$ in a given sample, with energy

$$
\mathcal{H}_{V}[u]=\int_{q} \frac{1}{2}\left(q^{2}+m^{2}\right) u(-q) \cdot u(q)+\int_{x} V(x, u(x)),
$$

where $\int_{q} \equiv \int \frac{\mathrm{d}^{d} q}{(2 \pi)^{d}}, \int_{x} \equiv \int \mathrm{d}^{d} x$ and $u \cdot v=\sum_{i=1}^{N} u^{i} v^{i}$. The $O(N)$ indices will be specified only when strictly necessary, and below additional replica indices for the replicated field $u_{a}^{i}$ will be introduced, $a=$ 
$1, \ldots, n$. The small confining mass $m$ provides a scale. To obtain a non-trivial large- $N$ limit, one defines the rescaled field $v=u / \sqrt{N}$ and chooses the distribution of the random potential to be rotationally invariant, e.g. its second cumulant as

$$
\overline{V(x, u) V\left(x^{\prime}, u^{\prime}\right)}=R\left(u-u^{\prime}\right) \delta_{x x^{\prime}}=N B\left(\left(v-v^{\prime}\right)^{2}\right) \delta_{x x^{\prime}}
$$

in terms of a function $B(z)$. Higher connected cumulants are scaled as

$$
{\overline{V\left(x_{1}, u_{1}\right) \ldots V\left(x_{p}, u_{p}\right)}}^{\mathrm{conn}}=N \delta_{x_{1}, \ldots, x_{p}} S^{(p)}\left(v_{1}, \ldots, v_{p}\right)
$$

with $\delta_{x_{1}, \ldots, x_{p}}:=\prod_{i=2}^{p} \delta^{d}\left(x_{1}-x_{i}\right)$.

Physical observables can be obtained for any $N$ from the replicated action at $n=0$ with a source $J=\sqrt{N} j$ as

$$
\begin{aligned}
\mathcal{Z}[J]= & \int \mathcal{D}[u] \mathcal{D}[\chi] \mathcal{D}[\lambda] \mathrm{e}^{-N \mathcal{S}[u, \chi, \lambda, j]} \\
\mathcal{S}[u, \chi, \lambda, j]= & \frac{1}{2 T} \int_{q}\left(q^{2}+m^{2}\right) v_{a}(-q) \cdot v_{a}(q) \\
& +\int_{x} U(\chi(x))-\frac{1}{2} i \lambda_{a b}(x)\left[\chi_{a b}(x)-v_{a}(x) \cdot v_{b}(x)\right]-j_{a}(x) \cdot v_{a}(x),
\end{aligned}
$$

where the replica matrix field $\chi(x) \equiv \chi_{a b}(x)$ has been introduced through a Lagrange multiplier matrix field $\lambda_{a b}(x)$. Here and below summations over repeated replica (and $O(N)$ ) indices $a, b=1, \ldots, n$ is implicit. The bare interaction matrix potential

$$
U(\chi)=-\frac{1}{2 T^{2}} \sum_{a b} B\left(\tilde{\chi}_{a b}\right)-\frac{1}{3 ! T^{3}} \sum_{a b c} S\left(\tilde{\chi}_{a b}, \tilde{\chi}_{b c}, \tilde{\chi}_{c a}\right)+\ldots
$$

depends only on the matrix

$$
\tilde{\chi}_{a b}:=\chi_{a a}+\chi_{b b}-\chi_{a b}-\chi_{b a}
$$

and has a cumulant expansion in terms of sums with higher numbers of replicas.

The effective action functional $\Gamma[u]$ is defined as the Legendre transform of $\mathcal{W}[J]=\ln \mathcal{Z}[J]$ and satisfies

$$
\Gamma[u]+\mathcal{W}[J]=\int J(x) \cdot u(x) .
$$

Since $\Gamma[u]$ defines the renormalized vertices, its zero-momentum limit defines the renormalized disorder, the quantity on which we focus here. Thus we only need the result (per unit volume) for a uniform configuration of the replica field $u_{a}(x)=u_{a}=\sqrt{N} v_{a}$, which takes the form:

$$
\tilde{\Gamma}(v):=\frac{1}{L^{d} N} \Gamma(u)=\frac{1}{2 T} m^{2} v_{a}^{2}+\tilde{U}(v v),
$$

where $v v$ stands for the matrix $v_{a} \cdot v_{b}$. This defines the renormalized disorder potential $\tilde{U}(v v)$ and, whenever it can be expanded, up to a constant,

$$
\tilde{U}(v v)=-\frac{1}{2 T^{2}} \sum_{a b} \tilde{B}\left(v_{a b}^{2}\right)-\frac{1}{3 ! T^{3}} \sum_{a b c} \tilde{S}\left(v_{a b}^{2}, v_{b c}^{2}, v_{c a}^{2}\right)+\ldots
$$


It defines the renormalized cumulants $\tilde{B}(z), \tilde{S}(\ldots)$ etc.. Here and in the following we denote

$$
v_{a b}:=v_{a}-v_{b}
$$

We aim at calculating the effective action up to terms of order $O\left(1 / N^{2}\right)$, i.e. the first two terms in the expansion:

$$
\tilde{U}(v v)=\tilde{U}^{0}(v v)+\frac{1}{N} \tilde{U}^{1}(v v)+O\left(1 / N^{2}\right) .
$$

Details of the calculation, as well as expressions for non-uniform fields are given in Appendix C. For the leading term we find, from a saddle-point evaluation [8]:

$$
\begin{aligned}
\tilde{U}^{0}(v v) & =U\left(\chi_{v}\right)+\frac{1}{2} \sum_{n=1}^{\infty} \frac{n}{n+1} I_{n+1} \operatorname{tr}\left[-2 T \partial_{\chi} U\left(\chi_{v}\right)\right]^{n+1} \\
I_{n} & :=\int_{k} \frac{1}{\left(k^{2}+m^{2}\right)^{n}} .
\end{aligned}
$$

The trace acts on replica matrices and $\chi_{v}$ satisfies the self-consistent equation

$$
\begin{aligned}
\chi_{v}^{a b} & =v_{a} v_{b}+T \int_{k} G_{v}^{a b}(k)=v_{a} v_{b}+T I_{1} \delta_{a b}+T \sum_{n=1}^{\infty} I_{n+1}\left(\left[-2 T \partial_{\chi} U\left(\chi_{v}\right)\right]^{n}\right)_{a b} \\
G_{v}(k) & =\left[\left(k^{2}+m^{2}\right) \delta+2 T \partial_{\chi} U\left(\chi_{v}\right)\right]^{-1} .
\end{aligned}
$$

Note that for $d<2$ no UV cutoff is necessary (apart for a constant term in the free energy), while for $2<d<4$ an UV cutoff is necessary (and implicit in the following) only for $I_{1}{ }^{1}$.

One also finds a compact and very useful self-consistent equation for the derivative of the zero-th order potential:

$$
\begin{aligned}
\partial_{a b} \tilde{U}^{0}(v v) & =\partial_{a b} U\left(\chi_{v}\right) \\
& =\partial_{a b} U\left(v v+T I_{1} \delta+T \sum_{n=1}^{\infty} I_{n+1}\left(-2 T \partial \tilde{U}^{0}(v v)\right)^{n}\right)
\end{aligned}
$$

Everywhere we denote by $\partial_{a b} U(\phi):=\partial_{\phi_{a b}} U(\phi)$ the simple derivative of the function $U(\phi)$ with respect to its matrix argument $\phi_{a b}$. (Note that $\partial_{a b} \tilde{U}(v v)$ is a first derivative of $\tilde{U}(v v)$ with respect to the matrix element $v_{a} \cdot v_{b}$.)

Next, from calculations of the fluctuations around the saddle point, one obtains the $1 / N$ correction, which can be expressed in terms of the zero-th order quantities as:

$$
\begin{aligned}
\tilde{U}^{1}(v v) & =\frac{1}{2} \int_{q} \operatorname{tr}\left(\ln \left[\delta_{a c} \delta_{b d}+2 T \partial_{\chi_{a b}} \partial_{\chi_{e f}} U\left(\chi_{v}\right)\left(T \Pi_{v}^{e f, c d}(q)+v_{e} G_{v}^{f c}(q) v_{d}+v_{f} G_{v}^{e d}(q) v_{c}\right)\right]\right) \\
\Pi_{v}^{e f, c d}(q) & =\int_{k} G_{v}^{e d}(k) G_{v}^{f c}(q-k) \\
G_{v}(k) & =\left[\left(k^{2}+m^{2}\right) \delta+2 T \partial_{v v} \tilde{U}^{0}(v v)\right]^{-1}
\end{aligned}
$$

${ }^{1}$ To obtain a correct continuum limit, $T$ therefore should be scaled as $T=\tilde{T} / \Lambda^{d-2}$, when $\Lambda$ is taken to infinity. 


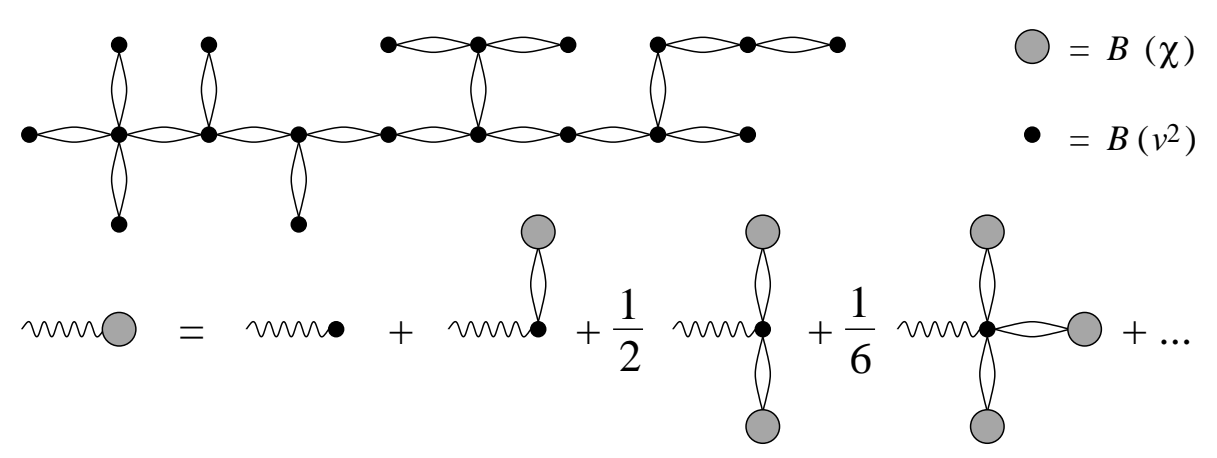

Figure 1: Top: typical $T=0$ contribution to $\tilde{B}\left(v_{a b}\right)$. Bottom: self-consistent equation at leading order for $\tilde{B}^{\prime}\left(v_{a b}^{2}\right)=B^{\prime}\left(\chi_{a b}\right)$. The wiggly line denotes a derivative, and is combinatorially equivalent to choosing one $B$. At finite $T$ one can attach an additional arbitrary number of tadpoles to any $B$. Also note that no loop made out of 3 propagators appears: this would be a contribution to the third cumulant (3-replica term), not calculated here; it is given in [8].

where here the trace acts in the space of replica pairs, i.e. $\operatorname{tr}(M)=\sum_{a b} M_{a b, a b}$. Note that $\tilde{U}^{0}(v v)$ can also be replaced by the full $\tilde{U}(v v)$ in the expression of $\tilde{U}^{1}(v v)$ with the same accuracy (i.e. at leading order in $1 / N)$.

The saddle-point equation (2.17) for the zero-th order and the result for the $1 / N$-correction (2.18) are still formal as they encode the full renormalized disorder distribution. To yield the renormalized disorder cumulants via (2.10),(2.12) they must be expanded in the number of replica sums, i.e. in cumulants. In the following Section 3, we recall the results for $N=\infty$, and proceed with the non-trivial evaluation of (2.18) via a graphical method in Section 4, and via an algebraic method in Section 5.

\section{Review of the results for $N=\infty$}

In this section we review the main results at $N=\infty$. Details can be found in [6].

\subsection{Self-consistent equation at $N=\infty$}

We start by recalling the cumulant expansion and only derive the result for the second cumulant. Higher cumulants are given in [6]. One studies a bare model, where only the second cumulant is non-zero:

$$
U(\chi)=-\frac{1}{2 T^{2}} \sum_{a b} B\left(\tilde{\chi}_{a b}\right)
$$

and calculates the renormalized disorder (2.10). (We will drop the index zero on the cumulant functions, indicating the leading order). The self-consistent equation (2.17) can be expanded in sums with increasing numbers of replicas. We only need:

$$
\begin{gathered}
{\left[-2 T \partial \tilde{U}^{0}(v v)\right]_{a b}=\frac{2}{T}\left(\delta_{a b} \sum_{c} \tilde{B}_{a c}^{\prime}-\tilde{B}_{a b}^{\prime}\right)+\ldots} \\
{\left[\left(-2 T \partial \tilde{U}^{0}(v v)\right)^{2}\right]_{a b}=\frac{4}{T^{2}}\left(\delta_{a b} \sum_{e f} \tilde{B}_{a e}^{\prime} \tilde{B}_{a f}^{\prime}-\tilde{B}_{a b}^{\prime} \sum_{f}\left(\tilde{B}_{a f}^{\prime}+\tilde{B}_{b f}^{\prime}\right)+\sum_{c} \tilde{B}_{a c}^{\prime} \tilde{B}_{c b}^{\prime}\right)+\ldots,}
\end{gathered}
$$

where $\tilde{B}_{a b}^{\prime}=\tilde{B}^{\prime}\left(v_{a b}^{2}\right)$ (recall $\left.v_{a b}^{2}:=\left(v_{a}-v_{b}\right)^{2}\right)$. Here and below the dropped terms contain sums with too many replicas to contribute to the final result for the self-consistent equation of the second cumulant 
(2-replica term). One thus has:

$$
\tilde{\chi}_{v}^{a b}=v_{a b}^{2}+2 T I_{1}\left(1-\delta_{a b}\right)+4 I_{2}\left[\frac{1}{2} \sum_{c}\left(\tilde{B}_{a c}^{\prime}+\tilde{B}_{b c}^{\prime}\right)-\frac{1}{2}\left(\tilde{B}_{a a}^{\prime}+\tilde{B}_{b b}^{\prime}\right)+\tilde{B}_{a b}^{\prime}-\delta_{a b} \sum_{c} \tilde{B}_{a c}^{\prime}\right]+\ldots
$$

The self-consistent equation becomes:

$$
\tilde{B}^{\prime}\left(v_{a b}^{2}\right)-\delta_{a b} \sum_{c} \tilde{B}^{\prime}\left(v_{a c}^{2}\right)+3 \text {-replica terms }=B^{\prime}\left(\tilde{\chi}_{v}^{a b}\right)-\delta_{a b} \sum_{c} B^{\prime}\left(\tilde{\chi}_{v}^{a c}\right)
$$

and can be solved by appropriate Taylor expansion of the r.h.s.. It is solved for $a \neq b$ :

$$
\tilde{B}^{\prime}\left(v_{a b}^{2}\right)+\frac{1}{T} \sum_{g} \tilde{S}_{a b g}^{\prime}+\sum_{g h} \ldots+\ldots=B^{\prime}\left(\tilde{\chi}_{v}^{a b}\right) .
$$

It is then easy to see that the second cumulant satisfies a closed equation at any $T$,

$$
\tilde{B}^{\prime}\left(v_{a b}^{2}\right)=B^{\prime}\left(v_{a b}^{2}+2 T I_{1}+4 I_{2}\left(\tilde{B}^{\prime}\left(v_{a b}^{2}\right)-\tilde{B}^{\prime}(0)\right)\right)
$$

with no other contributions from higher cumulants at any T. A more detailed derivation is given in [6].

\subsection{Derivation of the FRG equation at $N=\infty$}

From the previous section the renormalized second cumulant of the disorder $\tilde{B}^{\prime}(x)$ satisfies the selfconsistent equation

$$
\tilde{B}^{\prime}(x)=B^{\prime}\left(x+2 T I_{1}+4 I_{2}\left(\tilde{B}^{\prime}(x)-\tilde{B}^{\prime}(0)\right)\right)
$$

It implies

$$
\tilde{B}^{\prime}(0)=B^{\prime}\left(2 T I_{1}\right)
$$

as well as

$$
\tilde{B}^{\prime \prime}(x)=B^{\prime \prime}\left(x+2 T I_{1}+4 I_{2}\left(\tilde{B}^{\prime}(x)-\tilde{B}^{\prime}(0)\right)\right)\left[1+4 I_{2} \tilde{B}^{\prime \prime}(x)\right] .
$$

We now derive the corresponding exact FRG equation. Taking the derivative $m \partial_{m}$ gives:

$$
\begin{aligned}
m \partial_{m} \tilde{B}^{\prime}(x)= & B^{\prime \prime}\left(x+2 T I_{1}+4 I_{2}\left(\tilde{B}^{\prime}(x)-\tilde{B}^{\prime}(0)\right)\right) \\
& \times\left[2 T m \partial_{m} I_{1}+4\left(m \partial_{m} I_{2}\right)\left(\tilde{B}^{\prime}(x)-\tilde{B}^{\prime}(0)\right)+4 I_{2} m \partial_{m} \tilde{B}^{\prime}(x)-4 I_{2} m \partial_{m} \tilde{B}^{\prime}(0)\right] \\
= & \frac{\tilde{B}^{\prime \prime}(x)}{1+4 I_{2} \tilde{B}^{\prime \prime}(x)} \\
& \times\left[2 m \partial_{m} T I_{1}+4\left(m \partial_{m} I_{2}\right)\left(\tilde{B}^{\prime}(x)-\tilde{B}^{\prime}(0)\right)+4 I_{2} m \partial_{m} \tilde{B}^{\prime}(x)-4 I_{2} m \partial_{m} \tilde{B}^{\prime}(0)\right] .
\end{aligned}
$$

This yields:

$$
m \partial_{m} \tilde{B}^{\prime}(x)=\tilde{B}^{\prime \prime}(x)\left[2 m \partial_{m} T I_{1}+4\left(m \partial_{m} I_{2}\right)\left(\tilde{B}^{\prime}(x)-\tilde{B}^{\prime}(0)\right)-4 I_{2} m \partial_{m} \tilde{B}^{\prime}(0)\right]
$$

Thus one has also:

$$
m \partial_{m} \tilde{B}^{\prime}(0)=\frac{\tilde{B}^{\prime \prime}(0)}{1+4 I_{2} \tilde{B}^{\prime \prime}(0)} 2 m \partial_{m}\left(T I_{1}\right)
$$




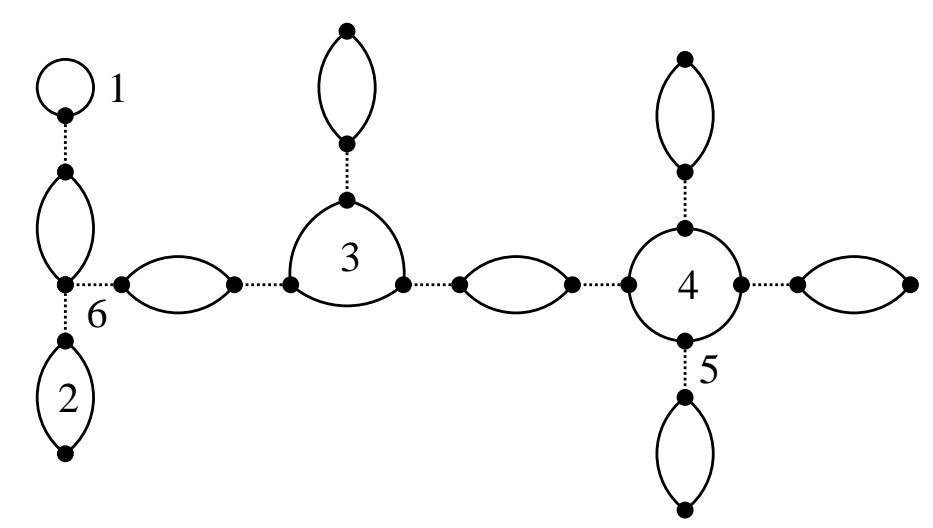

Figure 2: A typical "tree-like" diagram contributing to the leading order in the $1 / N$-expansion of the renormalized potential $\tilde{V}$ (effective action). The numbers 1,2 and 3 depict special features discussed in the main text. There is a factor $1 / N$ per dotted line and a factor of $N$ per "small loop" of propagators (solid lines), thus the overall factor is $N$ as it should from (4.1). This graph does not contain any "big loop" (see text).

Hence one gets finally

$$
m \frac{\partial}{\partial m} \tilde{B}^{\prime}(x)=\tilde{B}^{\prime \prime}(x)\left[2\left(m \frac{\partial}{\partial m} T I_{1}\right) \frac{1}{1+4 I_{2} \tilde{B}^{\prime \prime}(0)}+4\left(m \frac{\partial}{\partial m} I_{2}\right)\left(\tilde{B}^{\prime}(x)-\tilde{B}^{\prime}(0)\right)\right],
$$

which can also be integrated once over $x$. As emphasized in Ref. [6] it is exact at $N=\infty$ for any $d$ and correctly matches the 1-loop FRG equation obtained by Balents and Fisher for any $N$ but only to $O(\epsilon)$, $\epsilon=4-d$. It can be solved directly, or equivalently the self-consistent equation (3.8) can be inverted. The corresponding solutions for various models are discussed in [6] and compared with the Mézard-Parisi solution [11] obtained in a rather different manner through a replica-symmetry-breaking saddle point.

Before discussing specific models, we now turn to the evaluation of the effective action of the FRG and the $\beta$-function to the next order in $1 / N$.

\section{Corrections in $1 / N$, via the graphical method}

In this section, we present a graphical method to calculate the corrections to $\tilde{B}$ at order $1 / N$. An algebraic method is presented in the next section 5. Both methods are completely independent, since they use orthogonal ideas. They were performed independently by the authors, each on a different continent. The agreement on the final result gives some confidence that it is free from calculational errors.

\subsection{General considerations for a scalar field theory}

Let us start with some general considerations about which graphs contribute at a given order in $1 / N$. For that purpose, we consider a general (pure, no disorder) scalar field theory, with a $N$-component field $u^{i}(x)$, $i=1 \ldots N$ and interaction

$$
\mathcal{S}_{\text {int }}=N \int_{x} V\left(\frac{\vec{u}^{2}}{N}\right)
$$

where the $u u$-correlations of the free theory $(V=0)$ are given by

$$
\left\langle u^{i}(x) u^{j}(y)\right\rangle=\delta^{i j} C(x-y),
$$


with $i, j=1 \ldots N$ and $C(x-y)$ independent of $N$ is denoted by a solid line in figure 2 . Graphically, we can denote this by

$$
\left.S_{\mathrm{int}}=b_{2} \nmid+\frac{b_{4}}{N}\right\}\left\{\begin{array}{l}
b_{6} \\
N^{2}
\end{array}\right\} \nmid\{+\ldots,
$$

where all coefficients $b_{i}$ are of order 1 ; there is thus a factor of $1 / N$ per dotted line.

The renormalized potential $\tilde{V}$ (effective action) is given by the sum of all 1-particle-irreducible diagrams. They must thus contain "small loops". To leading order in $1 / N$ the following diagrams are possible:

- Tadpoles, contracting any $\vec{u}^{2}$ with itself only. The factor of $N$ from the "small loop" (the $\sum_{i=1}^{N}$ over the number of components) compensates the factor of $N$ from the argument of $V\left(\vec{u}^{2} / N\right)$. (See 1 on figure 2.)

- Closed "small loops" with 2, 3, 4 or more vertices, as denoted by the same number on figure 2: Adding one more vertex gives a factor of $N$, see Eq. (4.1); this is compensated by using an additional $\vec{u}^{2} / N$.

- Note that all vertices $\vec{u}^{2},\left(\vec{u}^{2}\right)^{2}$, a.s.o. contribute equivalently. (See 5, 6 on figure 2.)

Note that all these diagrams are "tree-like" diagrams, where the branches (made of "small loops") are made out of the diagrams through which no total momentum is running. We will call them "tree-like" in the following, to distinguish them from normal trees. They are resummed by the saddle-point equations (see appendix B) or from graphical inspection, as done here: First of all, insertions of $V^{\prime}$ into a line of propagators act like a mass, leading to the replacement of $1 /\left(k^{2}+m^{2}\right)$ by

$$
G(k):=\frac{1}{k^{2}+m^{2}+2 \tilde{V}^{\prime}\left(\frac{\vec{u}^{2}}{N}\right)} .
$$

The effective potential $\tilde{V}$ is obtained from

$$
\begin{aligned}
\tilde{V}^{\prime}\left(\frac{\vec{u}^{2}}{N}\right) & =V^{\prime}(\chi) \\
\chi & =\frac{\vec{u}^{2}}{N}+\int_{k} G(k)=\frac{u^{2}}{N}+\int_{k} \frac{1}{k^{2}+m^{2}+2 \tilde{V}^{\prime}\left(\frac{\vec{u}^{2}}{N}\right)} .
\end{aligned}
$$

Note that the derivatives are graphically understood as follows: Choosing one vertex (derivative!) in the effective potential $\tilde{V}$ is equivalent to having a bare vertex with the same derivative taken (thus $V^{\prime}$ ) and attaching to it loops made out of correlation-functions. Attaching any number of such loops to $V$, amounts to shifting its argument, as can be seen from Taylor-expansion. In these loops, again derivatives (one needs $u^{2}$ to attach the loop) of the effective potential are inserted. The latter can thus be written as $V^{\prime}$ with shifted argument to account for more things to be attached to this $V^{\prime}$ or equivalently using (4.5) to a $\tilde{V}^{\prime}$. This result coincides with (B.22).

Diagrams at next order $1 / N$ contain exactly one "big loop", see figure 3. Take a "tree" (as e.g. the object on Fig. 2) and glue it together to form a "big loop" by identifying two vertices; this does not change the factor of $N$ from the loops, but one looses one factor of $N$ from the missing vertex. Note that also the "minimal loop" marked on figure 3 belongs to the same class, even though it looks different. The "big loop" demands to sum a series of diagrams at non-vanishing momentum, and then to carry through the integration 


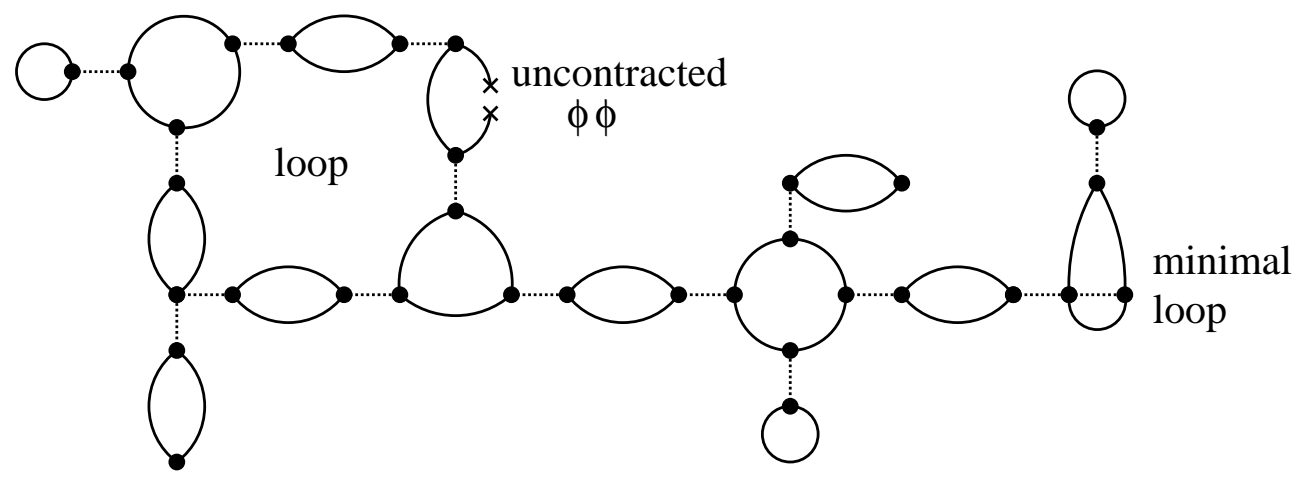

Figure 3: Typical graphs which contribute to the renormalized potential $\tilde{V}$ to subleading order in $1 / N$. The "big loop" (a loop made of loops, see main text) accounts for a factor of $1 / N$; the same is true for the "minimal loop" on the right, which is a "big loop" in disguise (as explained in the main text.) The given diagram is thus of order $1 / N^{2}$

over momenta. However the simplification remains that any added "tree-like" branches are resummed by replacing the argument of $V$ from $\vec{u}^{2} / N$ to $\chi$.

Another feature arises at subdominant order: In the "big" loop, one may pick any given small loop (i.e. the loop made out of correlation-functions) and replace one of the two correlation-functions by $\vec{u}^{2} / N:$ This means that the corresponding fields $u$ did not get contracted. Since the remaining correlation-functions force their indices to be equal, this gives a factor of $\vec{u}^{2}=N \times\left(\vec{u}^{2} / N\right)$, thus contributes the same factor of $N$. Note that these diagrams do not contribute to the effective action at leading order, which is treelike, since the resulting diagrams would be 1-particle reducible.

To resum the order $1 / N$-diagrams one has to sum over loops of all sizes. The result is

$$
\begin{aligned}
\delta \tilde{V}\left(\frac{\vec{u}^{2}}{N}\right) & =-\frac{1}{2 N} \sum_{n=1}^{\infty} \frac{1}{n} \int_{p}\left[-2 V^{\prime \prime}(\chi)\left(I_{2}(p)+2 \frac{\vec{u}^{2}}{N} G(p)\right)\right]^{n} \\
& =\frac{1}{2 N} \int_{p} \ln \left[1+2 V^{\prime \prime}(\chi)\left(I_{2}(p)+2 \frac{\vec{u}^{2}}{N} G(p)\right)\right] \\
I_{2}(p) & =\int_{k} G(k+p) G(k) .
\end{aligned}
$$

This can be compared to the results of appendix B, and more specifically to formula (B.39).

\subsection{Elastic manifolds in disorder: General considerations, building blocks}

Let us start the treatment of the disordered model with some general considerations. First to organize the $1 / N$-expansion, one may still use the diagrammatics of the previous section, which shows the $O(N)$-index content. The same diagrams still exist, but they now also have a complicated replica content. The replica content can be explicated by using "splitted vertices" instead of the unsplitted ones of the previous section. The corresponding replica diagrammatics, which shows the replica structure only was explained in details in [7]. This can be drawn as

$$
\sum_{a b} B\left(\left(v_{a}-v_{b}\right)^{2}\right)={ }_{a} \bullet \bullet b_{b}
$$

where a dashed line connects the two dots, standing for replicas $a$ and $b$. In order to avoid confusion, note that this dashed line is different from the dotted line used in figures 2 and 3 as well as equation (4.3) to show the $O(N)$-structure. 


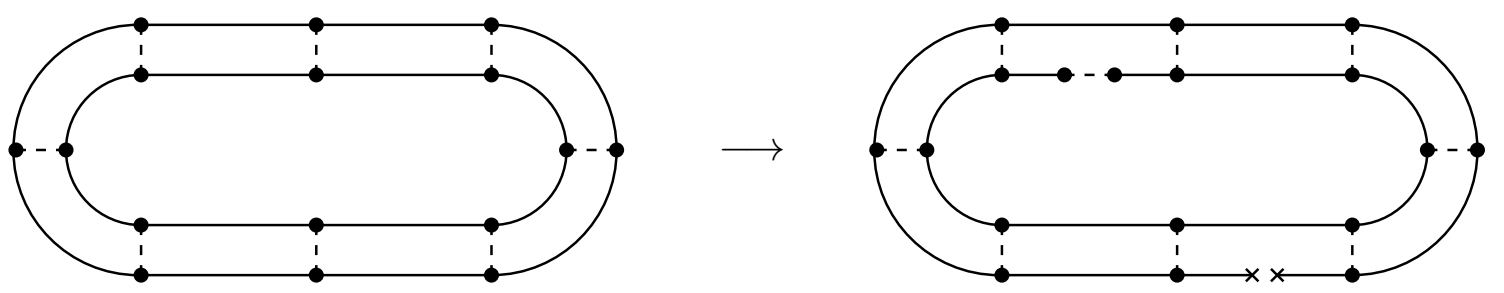

Figure 4: Example of a big loop made out of small loops, in splitted replica notation (left). It contributes to the 2-replica part (i.e. the disorder) at order $1 / N$. Note that there are two constraints more than needed to have a 2-replica term. Thus two redundant constraints can be "wasted", by cutting each of the solid lines exactly once, either by inserting a $B^{\prime}$ or leaving a $u u$ uncontracted, as is done on the right.

Below we introduce a third diagrammatics which allows to track both the replica and the $O(N)$ indices, not an easy task. Before doing so let us explain a few points.

Since we are only interested in the corrections to the 2-replica part $\tilde{B}_{a b}$ of the effective action, there are many $O(N)$ diagrams which do not contribute.

At dominant order (figure 1) small loops with three vertices (see 3 in figure 2), or more, do not contribute to $\tilde{B}_{a b}$, but to the third cumulant, or higher, as can be seen from

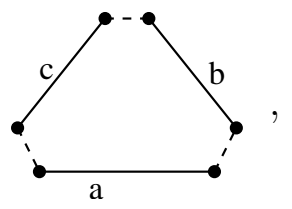

which is a diagram showing only replica indices, $N$ indices being implicit. Note that solid lines identify replica indices. This is why in figure 1 only chain-diagrams and tadpoles (the latter are omitted for simplicity of presentation) appear, rendering calculations appreciably simpler.

At subdominant order in $1 / N$, only diagrams with one "big" loop (general feature of the order $1 / N$ discussed in the last section) made of any number of "small" 2-loops and only exactly two "small" 3loops can contribute to the renormalized second cumulant. Each small 3-loop can also be replaced by an uncontracted $u$. To understand this, consider the simplest "big" loop, i.e. the railroad diagram, which is drawn on the left of figure 4 in splitted replica notation. It contains exactly two closed propagator lines, which over-constrain the replicas to be equal. (These are the inner and outer solid lines on the left of figure 4). These over-constraints can be relaxed, by cutting each line exactly once, in order not to get a higher replica term, as is illustrated on the right of figure 4. This "cutting" is possible by either inserting into a propagator a vertex (which contains two replicas, thus is not "replica-conserving") or by leaving one $u u$ uncontracted. This is the basic principle, whose careful exploration leads to all of the diagrams at order $1 / N$, as we will discuss now.

In order to do so, we have to introduce a new powerful graphical notation:

$$
\begin{aligned}
\text { - } & =B(\bar{\chi}) \\
\leftarrow^{a}{ }_{b} & =B^{\prime}\left(\bar{\chi}_{a b}\right)\left(\vec{u}_{a}-\vec{u}_{b}\right)^{2} \\
{ }_{b}^{a}{ }_{b}{ }_{b}^{a} & =B^{\prime \prime}\left(\bar{\chi}_{a b}\right) \frac{1}{2}\left[\left(\vec{u}_{a}-\vec{u}_{b}\right)^{2}\right]^{2},
\end{aligned}
$$

where lines departing in the same direction belong to the same vector-index, and a continuing line represents the same replica.

We also use the following short-hand notation

$$
B_{a b}^{\prime}:=B^{\prime}\left(\bar{\chi}_{a b}\right)
$$


and similar formulas for the higher derivatives $B_{a b}^{\prime \prime}:=B^{\prime \prime}\left(\bar{\chi}_{a b}\right)$, etc. Another frequently used shorthand is

$$
B_{0}^{\prime}:=B_{a a}^{\prime}, \quad B_{0}^{\prime \prime}:=B_{a a}^{\prime \prime}, \quad \text { etc. } .
$$

In order to be able to resum the "big" loop, we will now introduce some building blocks. Since there can be any number of 2-loops in the "big" loop one needs to define the resummed chain

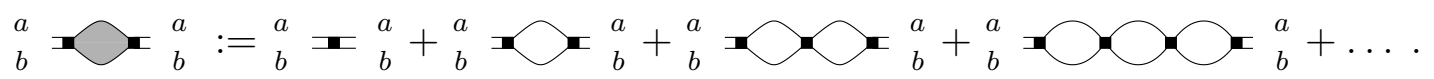

We have with momentum $p$ running through the diagram

$$
\stackrel{p}{\longrightarrow}{ }_{b}^{a} \bigcirc{ }_{b}^{a}=4 I_{2}(p) B^{\prime \prime}\left(\bar{\chi}_{a b}\right)_{b}^{a}={ }_{b}^{a}
$$

with

$$
I_{2}(p)=\int_{k} \frac{1}{(k+p / 2)^{2}+m^{2}} \frac{1}{(k-p / 2)^{2}+m^{2}} .
$$

Let us introduce a compact notation for the integrals, summarized in appendix I. (All our notations and important formulas are also summarized in a table in appendix J.)

$$
\begin{aligned}
J_{\alpha \beta}(q) & \equiv J_{\alpha \beta}^{q}:=\int_{k} \frac{1}{\left((k+q / 2)^{2}+m^{2}\right)^{\alpha}} \frac{1}{\left((k-q / 2)^{2}+m^{2}\right)^{\beta}} \\
I_{3}(p) & :=J_{1,2}(p)=\int_{k} \frac{1}{\left((k+p / 2)^{2}+m^{2}\right)^{2}} \frac{1}{(k-p / 2)^{2}+m^{2}} \\
I_{4}(p) & :=J_{2,2}(p)=\int_{k} \frac{1}{\left((k+p / 2)^{2}+m^{2}\right)^{2}} \frac{1}{\left((k-p / 2)^{2}+m^{2}\right)^{2}} .
\end{aligned}
$$

Thus

$$
\begin{aligned}
& \stackrel{p}{\longrightarrow}{ }_{b}^{a} \bigcirc{ }_{b}^{a}=\left[4 I_{2}(p) B^{\prime \prime}\left(\bar{\chi}_{a b}\right)\right]^{2}{ }_{b}^{a}={ }_{b}^{a} \\
& \stackrel{p}{\longrightarrow}{ }_{b}^{a} \bigcirc{ }_{b}^{a}=\left[4 I_{2}(p) B^{\prime \prime}\left(\bar{\chi}_{a b}\right)\right]^{3}{ }_{b}^{a}{ }_{b}^{a}{ }_{b}^{a},
\end{aligned}
$$

and so on. These chain-like diagrams form a geometric series, which is resummed as

$$
{ }_{b}^{a} \bigcirc{ }_{b}^{a}=\frac{1}{1-4 I_{2}(p) B^{\prime \prime}\left(\bar{\chi}_{a b}\right)}{ }_{b}^{a}=_{b}^{a}=: \frac{1}{2}\left[\left(u_{a}-u_{b}\right)^{2}\right] H_{a b}(p)\left[\left(u_{a}-u_{b}\right)^{2}\right] .
$$

We have introduced $H_{a b}(p)$, the "effective" $B_{a b}^{\prime \prime}$ after resummation

$$
H_{a b}(p):=\frac{B^{\prime \prime}\left(\bar{\chi}_{a b}\right)}{1-4 I_{2}(p) B^{\prime \prime}\left(\bar{\chi}_{a b}\right)},
$$

which we equivalently can express at leading order in $1 / N$ through $B^{\prime \prime}\left(\bar{\chi}_{a b}\right)=\tilde{B}_{a b}^{\prime \prime} /\left[1+4 I_{2} \tilde{B}_{a b}^{\prime \prime}\right]$ as

$$
H_{a b}(p):=\frac{\tilde{B}_{a b}^{\prime \prime}}{1+4\left[I_{2}-I_{2}(p)\right] \tilde{B}_{a b}^{\prime \prime}} .
$$




\begin{tabular}{|c|c|}
\hline - & $B\left(\bar{\chi}_{a b}\right)$ \\
\hline $\begin{array}{l}a \\
b\end{array}$ & $B^{\prime}\left(\bar{\chi}_{a b}\right)\left(\vec{u}_{a}-\vec{u}_{b}\right)^{2}$ \\
\hline $\begin{array}{l}a \\
b\end{array}=\begin{array}{l}a \\
b\end{array}$ & $B^{\prime \prime}\left(\bar{\chi}_{a b}\right) \frac{1}{2}\left[\left(\vec{u}_{a}-\vec{u}_{b}\right)^{2}\right]^{2}$ \\
\hline$a-$ & $\delta_{a b} C(p)=\frac{\delta_{a b}}{p^{2}+m^{2}}$ \\
\hline$a \multimap \times{ }_{b}$ & $v_{a b}^{2}$ \\
\hline $\begin{array}{l}a \\
b\end{array}$ & $H_{a b}(p)=\frac{B^{\prime \prime}\left(\bar{\chi}_{a b}\right)}{1-4 I_{2}(p) B^{\prime \prime}\left(\bar{\chi}_{a b}\right)}$ \\
\hline$a \curvearrowleft b$ & $\delta_{a b} I_{2}(p)\left(1-4 I_{2} B_{a a}^{\prime \prime}\right)$ \\
\hline$a \curvearrowleft a$ & $I_{2}(p)\left(1+2 I_{2} B_{a a}^{\prime \prime}\right)\left(1-4 I_{2} B_{a a}^{\prime \prime}\right)$ \\
\hline
\end{tabular}

Figure 5: Building blocks of the perturbation theory. See main text. The last two blocks only appear at finite temperature.

We also define $H_{v}(p)$ as

$$
H_{v}(p):=\frac{B^{\prime \prime}(\bar{\chi}(v))}{1-4 I_{2}(p) B^{\prime \prime}(\bar{\chi}(v))}=\frac{\tilde{B}^{\prime \prime}\left(v^{2}\right)}{1+4\left[I_{2}-I_{2}(p)\right] \tilde{B}^{\prime \prime}\left(v^{2}\right)},
$$

valid again at leading order in $1 / N$. Note that the denominator in (4.27) reflects the renormalization of $\tilde{B}$ : The divergent integral $I_{2}(p)$ does not appear alone, but together with its counter-term, the integral $I_{2}(p)$ subtracted at $p=0$. It turns out that at zero temperature the above are the only building blocks needed. However at $T>0$ one needs two more building blocks which are quite non-trivial. As was shown in [7] non-zero temperature diagrams contain at least one replica "sloop"; these are exactly the over-constraining lines discussed above and on figure 4. There is one factor of $T$ for each such "sloop". The additional building blocks thus contain sloops: One sloop at order $T$, and 2 sloops (an example is on the left of figure 4) at order $T^{2}$. Higher orders in $T$ are only possible at order $1 / N^{2}$, or higher.

To explain the construction of these additional building blocks, which is subtle, one goes back to the diagrammatics showing only replica indices.

The first building block is the "moon-diagram". This is the sum over all diagrams, which at both ends have lines joining only one of the both replicas, and which enforce the joined replicas to be equal:

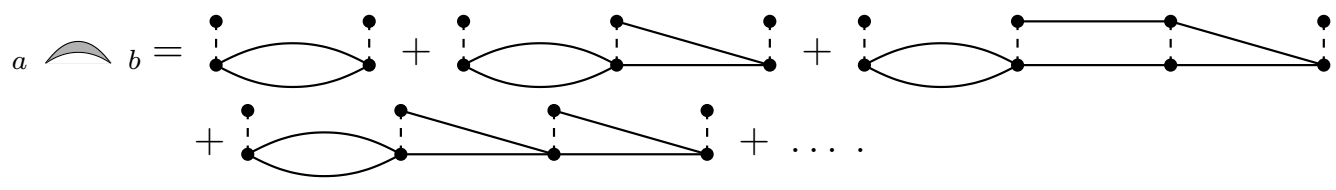

Note that we construct the chain from left to right. Otherwise the graphical representation of the perturbation expansion is not unambiguous, as can be seen from the following example

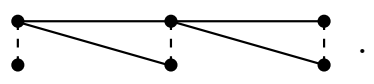


Drawing first the two left-most lines, the two right-most ones can no longer be added, since in the middle, there is $R^{\prime \prime}\left(u_{a}-u_{a}\right) \equiv R^{\prime \prime}(0)$, which does not depend on the field. Conversely, if we decided to first draw the two rightmost lines, then the two leftmost could be drawn. Consequently the diagrams to be drawn in (4.28) would be different (actually they would be nothing but the diagrams mirrored such that their left and right ends are exchanged), even though the final result would be the same. This phenomenon is detailed (for a different diagram) in appendix E. Note that there is nor a contradiction, nor an inconsistency of the approach. It merely means, that when using these kind of rules, which have the advantage of simplifying calculations importantly, one has to order the contractions. An approach, which does not have this deficiency, but is very complicated, is explained in appendix D. We will use it there to recalculate diagram (4.28).

We claim that

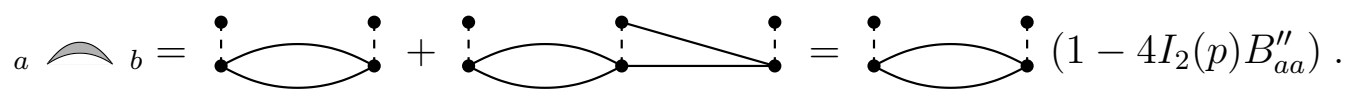

The second identity is trivial perturbation theory. The non-trivial statement is the first identity. To prove it, we remark that starting with (recall we construct from left to right) made. Therefore, we have to start with

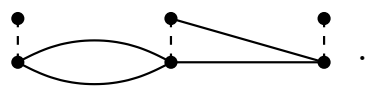

At chain-length three, there are two and only two possible prolongations, which have no additional free replica-indices:

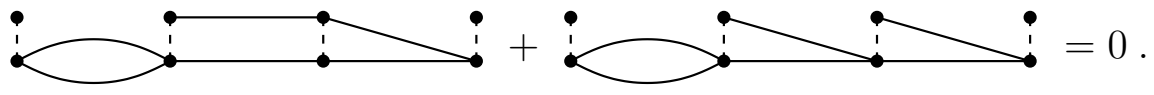

These diagrams cancel. The same is true for longer chains, since at any intermediate position (i.e. not the first and not the last lines), there is always the combination

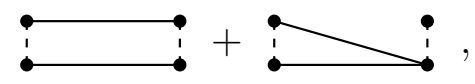

which when closed at the right end with

The last diagram which we need is the "half-moon-diagram" $\infty$, which is similar to the moondiagram, but does not enforce the replicas at its ends to be equal. However it will always be evaluated at coinciding replicas. (It thus contains $\frown$ as a subset.) We claim that

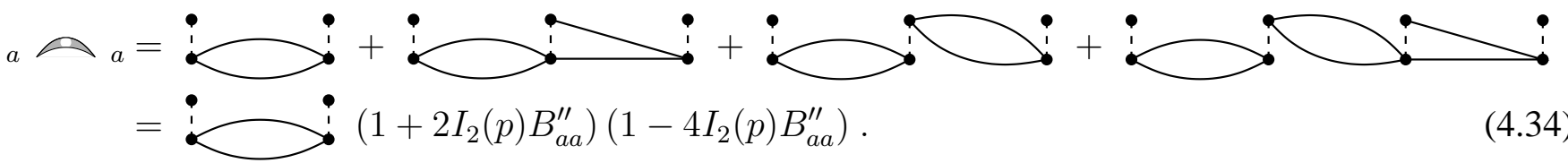

This is proven by first remarking that all other diagrams can be generated from those: Add left of a or a $:$ the combination

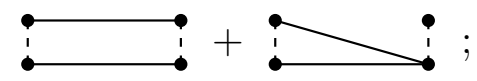

they cancel pairwise. The same is true for chains made out of the combination $:$ note that one cannot insert more

All rules and building blocks are collected on figure 5 . 


\subsection{Zero temperature $(T=0)$}

We start our discussion with zero temperature, $T=0$. We have to construct all diagrams with the topology of a loop in the large- $N$ limit. Note that e.g. the diagram (4.17) counts as a line in this construction. The building blocks are given on figure 5. At zero temperature, one needs all possible constraints on the sum of replicas. This means that one can not use $\frown$ or $\curvearrowright$, which both contain one non-replica conserving line $\vdots i$ at zero temperature. At finite temperature, one can use one at order $T$ and two at order $T^{2}$.

At $T=0$, we find the following diagrams. Note that the notation is such that crossing lines do not intersect. All the diagrams correct the effective action $\tilde{B}$ without any further combinatorial factor.

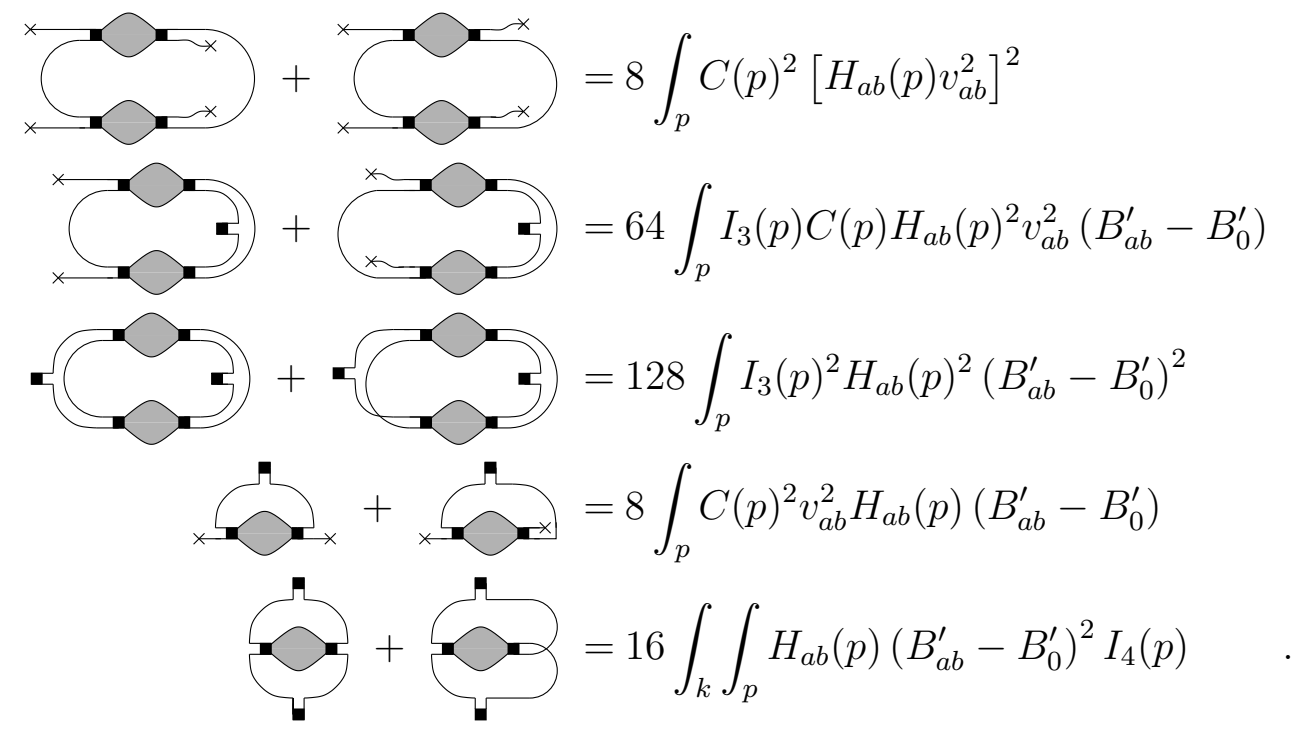

The combinatorial factors can, and have been checked by straightforward calculating the diagrams with $H_{a b}$ replaced by $B_{a b}^{\prime \prime}$, both by hand and computer-algebraically.

The idea of how to construct these diagrams is straightforward: We start by a closed chain of 2-loops, which disregarding the $O(N)$-structure has been drawn on the left of figure 4 . Then one has to cut each line exactly once. These cuts can either be done at different positions in the "big" loop (diagrams (4.36) to (4.38)), or at the same position (diagrams (4.39) and (4.40)). Then there is the possibility to either insert into a propagator a vertex (these are the terms proportional to $B_{a b}^{\prime}-B_{0}^{\prime}$ ) or not to contract two fields (the terms proportional to $v_{a b}^{2}$ ). The left and right diagrams in each equation are distinguished by their twist: The left one is the untwisted one (as the one drawn on the left of figure 4), the right one the twisted one, obtained by cutting both lines between two neighboring vertices, and reglueing them together with the two lines exchanged (this gives one single propagator line running twice around.) Note that the twisted diagrams do not appear in the final result, since we suppose analyticity for $B$, such that e.g. $\lim _{a \rightarrow b} H_{a b} v_{a b}^{2}=0 .^{2}$

As discussed above other imaginable contributions are 3-replica terms, where one is not using the maximal number of possible constraints on the number of free replica-sums (one line cut twice instead of each line cut once):

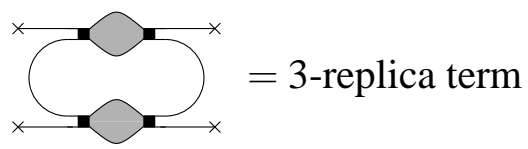

\footnotetext{
${ }^{2}$ Note that this construction suggests how to construct additional "anomalous" terms, known e.g. to be necessary at 2-loop order $[7,50]$. We do not present them here, but relegate their discussion to a subsequent publication.
} 


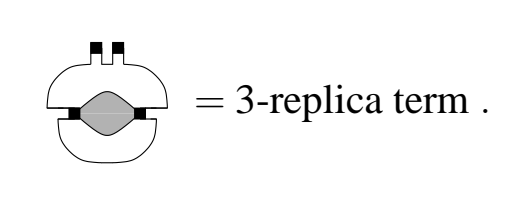

We note contributions which vanish identically for completeness:

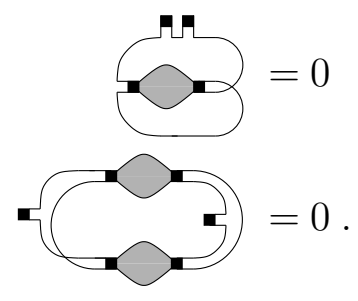

\subsection{Corrections at order $T$}

We remark that adding an additional line to any object already constructed at order $1 / N$ results into a diagram of higher topology in the large $N$-limit. This means that the diagram does not contribute, a statement which remains true to any order in perturbation theory. The only remaining possibility is to proceed as before, but constraining replica-indices to be the same by more than one propagator (line). Examples are $\frown$ and $\curvearrowright$. Another example would be a "circular railroad diagram", see left of figure 4 and equation (4.50) below. Since we had two lines to "waste", it means that there will be a term of order $T$ and $T^{2}$. (As a side-remark, we note that at order $1 / N^{2}$, there will be terms of up to order $T^{4}$, since for the leading term one has to cut up to 4 lines, a.s.o..)

We now give the order $T$-contributions:

$$
\begin{aligned}
& \bigcirc_{x}=4 T \int_{p} \frac{v_{a b}^{2} H_{a b}(p)}{p^{2}+m^{2}} \\
& =16 T \int_{p} I_{3}(p) H_{a b}(p)\left[B_{a b}^{\prime}-B_{0}^{\prime}\right] .
\end{aligned}
$$

These are the contributions, where one replica-line has been cut by the insertion of either $v^{2}$ or $B^{\prime}$, whereas the other one (on top of the diagrams) is redundant.

We can also use a double (redundant) line, using the moon diagram $\frown$. Starting from Eq. (4.45), and inserting it into the bubble line, we find

$$
\times=8 T \int_{p} C(p) v_{a b}^{2} H_{a b}(p)^{2} I_{2}(p)\left[1-4 I_{2}(p) B_{a a}^{\prime \prime}\right] .
$$

Starting the same procedure from (4.46) gives

$$
32 T \int_{p} H_{a b}(p)^{2}\left(B_{a b}^{\prime}-B_{a a}^{\prime}\right) I_{2}(p) I_{3}(p)\left[1-4 I_{2}(p) B_{a a}^{\prime \prime}\right] .
$$

Note that there is also a "twisted" version of (4.46) and (4.48), which add up to

$$
3+3=-16 T \int_{p} H_{a a}(p) B_{a b}^{\prime} I_{3}(p)\left[1+2 I_{2}(p) B_{a a}^{\prime \prime}\right] .
$$




\subsection{Corrections at order $T^{2}$}

We continue with diagrams at order $T^{2}$. There is one diagram, which does not necessitate any cut in a replica-conserving line, thus has two redundancies. It is the "railroad-diagram", i.e. a closed chain, with at least one vertex. Note that contrary to what one might expect, for one vertex this is not a product of two tadpoles summed at leading order, even though it looks alike. However, the structure in vector-indices makes it a loop in $N$, i.e. a subdominant term. Since no vertex is marked, the sum is not a geometric series, but a logarithm:

$$
\bigcirc=\frac{T^{2}}{2} \int_{p} \sum_{n=1}^{\infty} \frac{\left[4 B^{\prime \prime}\left(\bar{\chi}_{v}\right) I_{2}(p)\right]^{n}}{n} \text {. }
$$

This can be written either as a function of the bare disorder, or using $B^{\prime \prime}\left(\bar{\chi}_{a b}\right)=\tilde{B}_{a b}^{\prime \prime} /\left(1+4 I_{2} \tilde{B}_{a b}^{\prime \prime}\right)($ valid at all $T$ ) as a function of the renormalized disorder:

$$
\begin{aligned}
& =-\frac{T^{2}}{2} \int_{p} \ln \left[1-4 I_{2}(p) B^{\prime \prime}\left(\bar{\chi}_{v}\right)\right] \\
& =-\frac{T^{2}}{2} \int_{p} \ln \left(\frac{1+4 \tilde{B}^{\prime \prime}(v)\left[I_{2}-I_{2}(p)\right]}{1+4 \tilde{B}^{\prime \prime}(v) I_{2}}\right) .
\end{aligned}
$$

Note that this is the term where a double, completely replica-conserving line goes around.

More diagrams are possible, with one and two defects, i.e. replica-line cuttings, equivalent to inserting $\curvearrowright$ or $\curvearrowright$. Using only one cut, both outer indices are forced to be equal, and we have to insert $\curvearrowright$, calculated in (4.34):

$$
=2 T^{2} \int_{p} I_{2}(p) H_{a b}(p)\left[1+2 I_{2}(p) B_{a a}^{\prime \prime}\right]\left[1-4 I_{2}(p) B_{a a}^{\prime \prime}\right] .
$$

The overall prefactor of Eqs. (4.51) and (4.52) is such that the term linear in $\tilde{B}_{a b}^{\prime \prime}$ comes with a factor of 2, and they both add up to a factor of 4 , which can be checked with a simple 1-loop calculation.

The remaining term is obtained by cutting two-replica lines, using the replica-conserving moon $\frown$. This gives the contribution

$$
=2 T^{2} \int_{p}\left[I_{2}(p) H_{a b}(p)\left(1-4 I_{2}(p) B_{a a}^{\prime \prime}\right)\right]^{2} .
$$

There is an additional anomalous term. It is nothing but a 1-loop diagram, of the form $: \vdots$, where the right-most vertex is the sum of all diagrams at order $T^{2}$, as given by (4.50) to (4.53), evaluated at coinciding replicas:

$$
\begin{aligned}
\mathcal{A}^{T^{2}} & =-16 T^{2} I_{2} B_{a b}^{\prime} \int_{p} \frac{I_{2}(p)\left[1+2 I_{2}(p) B_{a a}^{\prime \prime}\right] B_{a a}^{\prime \prime \prime}}{1-4 I_{2}(p) B_{a a}^{\prime \prime}} \\
& =-16 T^{2} I_{2} B_{a b}^{\prime} \int_{p} I_{2}(p)\left[1+6 I_{2}(p) H_{a a}(p)\right] B_{a a}^{\prime \prime \prime} .
\end{aligned}
$$

This can be reexpressed as a function of $\tilde{B}$, see section 6.2. One might suspect that longer chains can be constructed to connect the $B_{a b}^{\prime}$ with the derivative of the effective action at order $T^{2}$, taken at coinciding indices. With the same arguments as already made a couple of times above, the insertions of $: \quad \vdots+$ 
$\vdots$ pairwise cancel. Also note that one could of course draw all these diagrams; there is however no clear advantage of doing so.

The alert reader will also wonder why we have not mentioned any such term at order $T^{0}$ or $T$. It turns out that the effective action at order $T^{0}$, when derived once and taken at coinciding arguments, actually vanishes (supposing analyticity!). At order $T$, the term in question is nothing but (4.49).

One caveat is in order: Even though this procedure is simple and elegant, there are many hidden traps. It is therefore good to check this calculation by an explicit loop expansion, using the excluded replica formalism. This has been done up to 8-loop order, and relies heavily on computer algebraic support. The procedure can also be formalized, leading to an additional more rigorous but somehow elaborate approach, the "Excluded Replica Formalism", which is presented in appendix D.

\section{Corrections at order $1 / N$, via the algebraic method}

The calculation of the renormalized disorder to next order requires the calculation of the following trace in the space of four replica matrices, from (2.18) and (2.19):

$$
\begin{aligned}
\tilde{U}^{1}(v v) & =\frac{1}{2} \int_{q} \operatorname{tr}\left[\ln \left(\delta_{a c} \delta_{b d}+\mathcal{M}_{a b, c d}\right)\right] \\
\mathcal{M}_{a b, c d} & =M_{a b, e f} N_{e f, c d}(q) \\
M_{a b, c d} & =\left.\left(2 T \partial_{\chi_{a b}} \partial_{\chi_{c d}} U(\chi)\right)\right|_{\chi=\chi_{v}} \\
\bar{N}_{e f, c d}(q) & =T \Pi_{v}^{e f, c d}(q)+G_{v}^{f c}(q) v_{e} \cdot v_{d}+G_{v}^{e d}(q) v_{f} \cdot v_{c} .
\end{aligned}
$$

$\Pi_{v}^{e f, c d}(q)$ has been defined in (2.19). Expression (5.1) can be computed by systematically expanding in sums over increasing numbers of replicas, as was done at dominant order. However the calculation is considerably more tedious in the present case. We give the main features here and relegate details to Appendix F.

To obtain the correction to the second cumulant of the disorder, we will only need the two-replica part of this function, which we denote $P_{2} \tilde{U}^{1}(v v)\left(P_{n} X\right.$ denotes the part of an expression $X$ which contains exactly $n$ free sums over replicas). Since the trace already involves at least one replica sum, we can and will truncate all expressions given below by discarding all terms with two or more replica sums. This can be checked systematically and originates from the fact that once a replica sum appears in an expansion, it can never disappear later on. As a result, we find that third and higher cumulants do not appear in the correction to the second cumulant, as they involve higher replica sums ${ }^{3}$.

The expansion of the matrices $M, \bar{N}$ and $\mathcal{M}$ are computed in Appendix F. It is crucial to write explicitly all Kronecker-delta functions. The matrix $\mathcal{M}$ is found to have the form:

$$
\mathcal{M}_{a b, c d}=m_{a b} \frac{1}{2}\left(\delta_{a d} \delta_{b c}+\delta_{b d} \delta_{a c}\right)+\left(1+m_{a b}\right) \overline{\mathcal{M}}_{a b, c d}
$$

where $m_{a b}$ is symmetric in $a, b$ and $\overline{\mathcal{M}}$ is symmetric in $a, b$ and symmetric in $c, d$ (but not necessarily in exchange of $(a, b)$ with $(c, d))$ and can thus be parameterized as

$$
\begin{aligned}
\overline{\mathcal{M}}_{a b, c d}= & \delta_{a b c d} x_{a}+\delta_{a b c} y_{a d}+\delta_{a b d} y_{a c}+\delta_{a c d} z_{a b}+\delta_{b c d} z_{b a}+\delta_{a c} t_{a b d}+\delta_{a d} t_{a b c}+\delta_{b c} t_{b a d}+\delta_{b d} t_{b a c} \\
& +\delta_{a b} \delta_{c d} u_{a c}+\delta_{a b} v_{a c d}+\delta_{c d} w_{a b c}+g_{a b c d},
\end{aligned}
$$

\footnotetext{
${ }^{3}$ This formal expansion in replica sums assumes some analyticity property in a way which should be analyzed later.
} 
where all Kronecker-delta's have been written explicitly. Also note that $v_{a c d}$ is symmetric in $c$ and $d ; w_{a b c}$ is symmetric in $a$ and $b$ and $g_{a b c d}$ is symmetric in $a, b$ and symmetric in $c, d$, whereas all others have no such symmetry. These matrices form a closed algebra which is studied in Appendix F. Unfortunately this algebra is rather large, even though it is the smallest algebra sufficient for the present calculation. Note that we have explicitly separated the part proportional to the (symmetrized) identity $1_{a b, c d}^{\mathrm{sym}}=1 / 2\left(\delta_{a c} \delta_{b d}+\delta_{a d} \delta_{b c}\right)$.

The first preliminary step in the trace $\log$ calculation is to prove $e^{4}$ that

$$
\left.\operatorname{tr}\left[\ln \left(\delta_{a c} \delta_{b d}+\mathcal{M}_{a b, c d}\right)\right]=\frac{1}{2} \sum_{a b} \ln \left(1+m_{a b}\right)+\frac{1}{2} \sum_{a} \ln \left(1+m_{a a}\right)+\operatorname{tr}\left[\ln \left(\delta_{a c} \delta_{b d}+\overline{\mathcal{M}}_{a b, c d}\right]\right)\right],
$$

where in the first two terms the $\ln$ simply acts on numbers. This formula is valid ${ }^{5}$ for a matrix $\mathcal{M}$ symmetric in $a, b$ and symmetric in $c, d$. The last trace log is equal to its usual series expression $\sum_{p>1} \frac{(-1)^{p+1}}{p}\left(\overline{\mathcal{M}}^{q}\right)_{a b, a b}^{p}$.

It does not seem possible to express the trace log in general but here it is possible to expand it systematically in the number of replica sums. Let us sketch the method that we found most convenient. We write

$$
\begin{aligned}
\operatorname{tr}[\ln (1+\overline{\mathcal{M}})] & =-\int_{0}^{1} \frac{\mathrm{d} \lambda}{\lambda} \operatorname{tr} M^{\lambda} \\
M^{\lambda} & =(1+\lambda \overline{\mathcal{M}})^{-1}-1,
\end{aligned}
$$

and we want to compute all terms of $M^{\lambda}$, namely:

$$
\begin{aligned}
M_{a b, c d}^{\lambda}= & \delta_{a b c d} x_{a}^{\lambda}+\delta_{a b c} y_{a d}^{\lambda}+\delta_{a b d} y_{a c}^{\lambda}+\delta_{a c d} z_{a b}^{\lambda}+\delta_{b c d} z_{b a}^{\lambda}+\delta_{a c} t_{a b d}^{\lambda}+\delta_{a d} t_{a b c}^{\lambda}+\delta_{b c} t_{b a d}^{\lambda}+\delta_{b d} t_{b a c}^{\lambda} \\
& +\delta_{a b} \delta_{c d} u_{a c}^{\lambda}+\delta_{a b} v_{a c d}^{\lambda}+\delta_{c d} w_{a b c}^{\lambda}+g_{a b c d}^{\lambda} .
\end{aligned}
$$

We do this by using the algebra detailed in Appendix F to solve the following equation, equivalent to (5.9):

$$
\lambda \overline{\mathcal{M}}+M^{\lambda}+\lambda \overline{\mathcal{M}} M^{\lambda}=0 .
$$

One projects onto each component $x, y, z, \ldots$ and onto terms with an increasing number of replica sums. What we want are $x^{\lambda}, y^{\lambda}, z^{\lambda}, \ldots$ as a function of the known $x, y, z, \ldots$, which parameterize $\overline{\mathcal{M}}$ (their expressions are given below).

More specifically one writes:

$$
\begin{aligned}
& x_{a}=P_{0} x_{a}+P_{1} x_{a}+\ldots \\
& x_{a}^{\lambda}=P_{0} x_{a}^{\lambda}+P_{1} x_{a}^{\lambda}+\ldots
\end{aligned}
$$

and similarly for all other components $y, z, t, \ldots$ of the matrices $\overline{\mathcal{M}}$ and $M^{\lambda}$. Using the algebraic rules for the product of two matrices, it turns out that it is possible to solve (5.11) for all components of $M^{\lambda}$ in an iterative manner. This is simplified since $P_{0} x_{a}=0$ (see below). First we determine all zero-sum components $P_{0} x^{\lambda}, P_{0} y^{\lambda}, \ldots$ from the corresponding $P_{0} x, P_{0} y, \ldots$ It can be done in the following order:

\footnotetext{
${ }^{4}$ Proof: Write $\mathcal{M}=\mathbb{A}+\left(\mathbb{1}+\mathbb{A}^{\prime}\right) \overline{\mathcal{M}}$, where $\mathbb{1}_{a b, c d}=\delta_{a c} \delta_{b d} . \quad$ Then $\operatorname{tr}[\ln (\mathbb{1}+\mathcal{M})]=\operatorname{tr}[\ln (\mathbb{1}+\mathbb{A}+(\mathbb{1}+$ $\left.\left.\left.\mathbb{A}^{\prime}\right) \overline{\bar{M}}\right)\right]=\ln \left(\operatorname{det}\left[\mathbb{1}+\mathbb{A}+\left(\mathbb{1}+\mathbb{A}^{\prime}\right) \overline{\mathcal{M}}\right]\right)=\ln (\operatorname{det}[\mathbb{1}+\mathbb{A}])+\ln \left(\operatorname{det}\left[\mathbb{1}+(\mathbb{1}+\mathbb{A})^{-1}\left(\mathbb{1}+\mathbb{A}^{\prime}\right) \overline{\mathcal{M}}\right]\right)$. Further $\ln (\operatorname{det}[\mathbb{1}+\mathbb{A}])=$ $\operatorname{tr}[\ln (1+\mathbb{A})]=\frac{1}{2} \sum_{a b} \ln \left(1+m_{a b}\right)+\frac{1}{2} \sum_{a} \ln \left(1+m_{a a}\right)$. Since $\overline{\mathcal{M}}_{a b, c d}=\overline{\mathcal{M}}_{b a, c d}$, the following identity holds: $\mathbb{A} \cdot \overline{\mathcal{M}}=\mathbb{A}^{\prime} \overline{\mathcal{M}}$, such that $\ln \left(\operatorname{det}\left[\mathbb{1}+(\mathbb{1}+\mathbb{A})^{-1}\left(\mathbb{1}+\mathbb{A}^{\prime}\right) \overline{\mathcal{M}}\right]\right)=\ln (\operatorname{det}[\mathbb{1}+\overline{\mathcal{M}}])=\operatorname{tr}[\ln (\mathbb{1}+\overline{\mathcal{M}})]=-\sum_{p>0}(-1)^{p} \operatorname{tr}\left[\overline{\mathcal{M}}^{p}\right]$. q.e.d.

${ }^{5}$ Note that the naive identity $\operatorname{tr}\left[\ln \left(\delta_{a c} \delta_{b d}+\mathcal{M}_{a b, c d}\right)\right]=\operatorname{tr}\left[\ln \left(\frac{1}{2}\left(\delta_{a c} \delta_{b d}+\delta_{a d} \delta_{b c}\right)+\mathcal{M}_{a b, c d}\right)\right]$ is incorrect.
} 
First compute $P_{0} y_{a b}^{\lambda}$ together with $P_{0} y_{a a}^{\lambda}$, then similarly $P_{0} z^{\lambda}, P_{0} x^{\lambda}, P_{0} t^{\lambda}, P_{0} v^{\lambda}, P_{0} w^{\lambda}, P_{0} g^{\lambda}$. Second one can project (5.11) onto one-sum terms, and determine $P_{1} x^{\lambda}, P_{1} y^{\lambda}$ etc...

At then end we need:

$$
\begin{aligned}
P_{2} \operatorname{tr} \ln [1+\overline{\mathcal{M}}]= & -\int_{0}^{1} \frac{\mathrm{d} \lambda}{\lambda} P_{2}\left(\operatorname{tr} M^{\lambda}\right) \\
P_{2}\left(\operatorname{tr} M^{\lambda}\right)= & \sum_{a}\left(P_{1} x_{a}^{\lambda}+2 P_{1} y_{a a}^{\lambda}+2 P_{1} z_{a a}^{\lambda}+2 P_{1} t_{a a a}^{\lambda}+P_{1} u_{a a}^{\lambda}+P_{1} v_{a a a}^{\lambda}+P_{1} w_{a a a}^{\lambda}\right) \\
& +\sum_{a b}\left(2 P_{0} t_{a b b}^{\lambda}+P_{0} g_{a b a b}^{\lambda}\right) .
\end{aligned}
$$

The remaining integrations over $\lambda$ of each term are found to be of the form $\int_{0}^{1} \mathrm{~d} \lambda \lambda^{p} /\left(1+2 y_{a a} \lambda\right)^{q}=$ ${ }_{2} F_{1}\left(1+p, q, 2+p,-2 y_{a a}\right) /(1+p)$ which for integer $p$ and $q$ can be expressed as rational fractions.

The detailed calculation is however very tedious and has been performed using Mathematica. The result is given in the following and is found to agree exactly with the graphical method of the previous section. Here we give the zero-sum and one-sum components of the matrix $\overline{\mathcal{M}}$ needed for the calculation of $P_{2} \operatorname{tr} \ln (1+\overline{\mathcal{M}})$. They are calculated from (5.1), see Appendix F, and read:

$$
\begin{aligned}
P_{0} x_{a}= & 0 \quad, \quad P_{0} u_{a c}=-P_{0} y_{a c} \quad, \quad P_{0} w_{a c d}=0 \\
P_{0} y_{a d}= & I_{2}(q) A_{a a}^{q} \bar{B}_{a d}^{\prime \prime} \quad, \quad A_{a a}^{q}=\frac{2}{1-4 B^{\prime \prime}\left(2 T I_{1}\right) I_{2}(q)} \\
P_{0} z_{a b}= & I_{2}(q) A_{a b}^{q} \bar{B}_{a b}^{\prime \prime} \quad, \quad A_{a b}^{q}=\frac{2}{1-4 \bar{B}_{a b}^{\prime \prime} I_{2}(q)} \\
P_{0} t_{a b d}= & -\frac{1}{T} A_{a b}^{q} \bar{B}_{a b}^{\prime \prime}\left[\left(v_{b}-v_{a}\right) v_{d} C(q)-2 I_{3}(q)\left(\tilde{B}_{b d}^{\prime}-\tilde{B}_{a d}^{\prime}\right)\right] \\
P_{0} v_{a c d}= & -\frac{1}{T} A_{a a}^{q}\left\{\bar{B}_{a d}^{\prime \prime}\left[\left(v_{d}-v_{a}\right) v_{c} C(q)-2 I_{3}(q)\left(\tilde{B}_{d c}^{\prime}-\tilde{B}_{a c}^{\prime}\right)\right]\right. \\
\left.\quad+\bar{B}_{a c}^{\prime \prime}\left[\left(v_{c}-v_{a}\right) v_{d} C(q)-2 I_{3}(q)\left(\tilde{B}_{c d}^{\prime}-\tilde{B}_{a d}^{\prime}\right)\right]\right\} & \left.\left.2 \tilde{B}_{b d}-\tilde{B}_{a d}^{\prime}\right) v_{c}\right] \\
P_{0} g_{a b c d}= & \frac{2}{T^{2}} C(q)^{2} A_{a b}^{q} \bar{B}_{a b}^{\prime \prime}\left(v_{a}-v_{b}\right)\left[\left(\tilde{B}_{b c}^{\prime}-\tilde{B}_{a c}^{\prime}\right) v_{d}+\left(\tilde{B}_{b c}^{\prime} \tilde{B}_{b c}\right.\right. \\
& -\frac{4}{T^{2}} I_{4}(q) A_{a b}^{q} \bar{B}_{a b}^{\prime \prime}\left(\tilde{B}_{a d}^{\prime} \tilde{B}_{b c}^{\prime}+\tilde{B}_{b d}^{\prime} \tilde{B}_{a c}^{\prime}-\tilde{B}_{a d}^{\prime} \tilde{B}_{a c}^{\prime}-\tilde{B}_{b d}^{\prime} \tilde{B}_{b c}^{\prime}\right)
\end{aligned}
$$

where we have defined $\tilde{B}_{a b}^{\prime}=\tilde{B}^{\prime}\left(v_{a b}^{2}\right)$ and

$$
\begin{aligned}
& \bar{B}_{a b}^{\prime \prime}=B^{\prime \prime}\left(\bar{\chi}_{a b}\right), \quad \bar{B}_{a b}^{\prime \prime \prime}=B^{\prime \prime \prime}\left(\bar{\chi}_{a b}\right) \\
& \bar{\chi}_{v}^{a b}=v_{a b}^{2}+2 T I_{1}+4 I_{2}\left(\tilde{B}_{a b}^{\prime}-\frac{1}{2}\left(\tilde{B}_{a a}^{\prime}+\tilde{B}_{b b}^{\prime}\right)\right)
\end{aligned}
$$

with $\bar{B}_{a a}^{\prime \prime}=B^{\prime \prime}\left(2 T I_{1}\right)$. All integrals $I_{n}$ and $I_{n}(q)$ are defined in section 4.2 and Appendix I and $C(q)=$ $1 /\left(q^{2}+m^{2}\right)$. We recall that $B$ is the bare second cumulant, $\tilde{B}$ the renormalized one and satisfies $\tilde{B}_{a b}^{\prime}=$ $B^{\prime}\left(\bar{\chi}_{v}^{a b}\right)$ at dominant order, which is sufficient for our purpose. For convenience we use $\bar{\chi}_{v}^{a b}:=\tilde{\chi}_{a b}^{(0)}$ to denote the zero sum part in the decomposition $\tilde{\chi}_{v}^{a b}=\tilde{\chi}_{a b}+\delta_{a b} \chi_{a}$ in the notations of [6].

For the 1-sum terms we need only the diagonal values:

$$
P_{1} x_{a}=-I_{2}(q) A_{a a}^{q} \sum_{f} \bar{B}_{a f}^{\prime \prime}
$$




$$
\begin{aligned}
P_{1} y_{a a}= & \frac{8}{T} \frac{1}{\left[1-4 B^{\prime \prime}\left(2 T I_{1}\right) I_{2}(q)\right]^{2}}\left[B^{\prime \prime}\left(2 T I_{1}\right) I_{3}(q)+T B^{\prime \prime \prime}\left(2 T I_{1}\right) I_{2} I_{2}(q)\right] \sum_{f} \tilde{B}_{a f}^{\prime} \\
& -\frac{2}{T} \frac{1}{1-4 B^{\prime \prime}\left(2 T I_{1}\right) I_{2}(q)} \sum_{f} \bar{B}_{a f}^{\prime \prime}\left[C(q)\left(v_{a}-v_{f}\right) v_{a}-2 I_{3}(q)\left(\tilde{B}^{\prime}(0)-\tilde{B}_{a f}^{\prime}\right)\right] \\
P_{1} z_{a a}= & -P_{1} u_{a a}=\frac{8}{T} \frac{1}{\left[1-4 B^{\prime \prime}\left(2 T I_{1}\right) I_{2}(q)\right]^{2}}\left[B^{\prime \prime}\left(2 T I_{1}\right) I_{3}(q)+T B^{\prime \prime \prime}\left(2 T I_{1}\right) I_{2} I_{2}(q)\right] \sum_{f} \tilde{B}_{a f}^{\prime} \\
P_{1} v_{a a a}= & -\frac{8}{T^{2}} \frac{1}{1-4 B^{\prime \prime}\left(2 T I_{1}\right) I_{2}(q)}\left[I_{4}(q) \sum_{f} \bar{B}_{a f}^{\prime \prime}\left(\tilde{B}_{a f}^{\prime}-\tilde{B}^{\prime}(0)\right)^{2}\right. \\
& \left.+C(q)^{2} \sum_{f} \bar{B}_{a f}^{\prime \prime}\left(\tilde{B}_{a f}^{\prime}-\tilde{B}^{\prime}(0)\right)\left(v_{a}-v_{f}\right) v_{a}\right] .
\end{aligned}
$$

In addition since $t_{a a a}=0$ one has $P_{1} t_{a a a}=0 ; P_{1} w_{a a a}=0$ since $w_{a b c}=0$ and it turns out that $P_{1} g_{a a a a}$ is not needed.

Starting from these values one performs the algebra and obtains $P_{2} \operatorname{tr} \ln (1+\overline{\mathcal{M}})$. To this one must add the "simple" part of $P_{2} \operatorname{tr} \ln$ in (5.7) above which one expands as follows:

$$
\begin{aligned}
& P_{2}\left[\frac{1}{2} \sum_{a b} \int_{q} \operatorname{tr} \ln \left(1+m_{a b}\right)+\frac{1}{2} \sum_{a} \int_{q} \operatorname{tr} \ln \left(1+m_{a a}\right)\right] \\
& =\frac{1}{2} \sum_{a b} \int_{q} \ln \left(1-4 \bar{B}_{a b}^{\prime \prime} I_{2}(q)\right)-\frac{8}{T} \int_{q} \frac{1}{1-4 B^{\prime \prime}\left(2 T I_{1}\right) I_{2}(q)}\left[I_{3}(q) B^{\prime \prime}\left(2 T I_{1}\right)+T I_{2} B^{\prime \prime \prime}\left(2 T I_{1}\right) I_{2}(q)\right] \tilde{B}_{a f}^{\prime},
\end{aligned}
$$

where $m_{a b}$ is computed in appendix F.

To translate the results into terms of the renormalized disorder, we can perform the replacements

$$
\begin{aligned}
\bar{B}_{a b}^{\prime \prime} & =\tilde{B}_{a b}^{\prime \prime} /\left(1+4 I_{2} \tilde{B}_{a b}^{\prime \prime}\right) \\
B^{\prime \prime}\left(2 T I_{1}\right) & =\tilde{B}^{\prime \prime}(0) /\left(1+4 I_{2} \tilde{B}^{\prime \prime}(0)\right) \\
\bar{B}_{a b}^{\prime \prime \prime} & =\tilde{B}_{a b}^{\prime \prime \prime} /\left(1+4 I_{2} \tilde{B}_{a b}^{\prime \prime}\right)^{3} \\
B^{\prime \prime \prime}\left(2 T I_{1}\right) & =\tilde{B}^{\prime \prime \prime}(0) /\left(1+4 I_{2} \tilde{B}^{\prime \prime}(0)\right)^{3},
\end{aligned}
$$

since to the same accuracy we can use in all above expressions the dominant order or the exact one.

The "simple" part of $P_{2} \operatorname{tr}$ ln thus gives:

$$
\begin{aligned}
\delta^{(\text {simple })} \tilde{B}(x)=\frac{8 T}{N} & \int_{p} I_{3}(p) H_{0}(p) \tilde{B}^{\prime}(x)+\frac{T^{2}}{N}\left\{-\frac{1}{2} \int_{p} \ln \left(\frac{1+4 \tilde{B}^{\prime \prime}(x)\left[I_{2}-I_{2}(p)\right]}{1+4 \tilde{B}^{\prime \prime}(x) I_{2}}\right)\right. \\
& \left.+8 I_{2} \int_{p} I_{2}(p) \tilde{B}^{\prime}(x) \frac{\tilde{B}^{\prime \prime \prime}(0)}{\left(1+4 I_{2} \tilde{B}^{\prime \prime}(0)\right)^{2}\left(1+4\left(I_{2}-I_{2}(q)\right) \tilde{B}^{\prime \prime}(0)\right)}\right\},
\end{aligned}
$$

where integrals are defined in Appendix I.

\section{Results for the effective action}

The $1 / N$ correction to the 2-replica part of the effective action in terms of the bare disorder can be written as:

$$
P_{2} \tilde{U}^{1}(v v)=-\frac{1}{2 T^{2}} \sum_{a b} \delta \tilde{B}\left(v_{a b}^{2}\right)
$$


with $v_{a b}^{2}=\left(v_{a}-v_{b}\right)^{2}$. It can be read off from sections 4.3, 4.4 and 4.5. We will give the expression for the correction to the renormalized second cumulant $\delta \tilde{B}(x)$ both in terms of the bare disorder and the renormalized one.

\subsection{The effective action as a function of the bare disorder}

We find:

$$
\begin{aligned}
& \delta \tilde{B}(v v)=\frac{1}{N}\left[8 \int_{p} C(p)^{2}\left[H_{v}(p) v^{2}\right]^{2}\right. \\
& +64 \int_{p} I_{3}(p) C(p) H_{v}(p)^{2} v^{2}\left[B^{\prime}\left(\bar{\chi}_{v}\right)-B^{\prime}\left(\bar{\chi}_{0}\right)\right] \\
& +128 \int_{p} I_{3}(p)^{2} H_{v}(p)^{2}\left[B^{\prime}\left(\bar{\chi}_{v}\right)-B^{\prime}\left(\bar{\chi}_{0}\right)\right]^{2} \\
& +8 \int_{p} C(p)^{2} v^{2} H_{v}(p)\left[B^{\prime}\left(\bar{\chi}_{v}\right)-B^{\prime}\left(\bar{\chi}_{0}\right)\right] \\
& \left.+16 \int_{p} H_{v}(p)\left[B^{\prime}\left(\bar{\chi}_{v}\right)-B^{\prime}\left(\bar{\chi}_{0}\right)\right]^{2} I_{4}(p)\right] \\
& +\frac{T}{N}\left[4 \int_{p}\left[C(p) v^{2}+4 I_{3}(p)\left(B^{\prime}\left(\bar{\chi}_{v}\right)-B^{\prime}\left(\bar{\chi}_{0}\right)\right)\right] H_{v}(p)\left[1+2 I_{2}(p) H_{v}(p)\right]\right. \\
& -32 \int_{p} I_{2}(p)^{2}\left[C(p) v^{2}+4 I_{3}(p)\left(B^{\prime}\left(\bar{\chi}_{v}\right)-B^{\prime}\left(\bar{\chi}_{0}\right)\right)\right] H_{v}(p)^{2} B^{\prime \prime}\left(\bar{\chi}_{0}\right) \\
& \left.-16 \int_{p} I_{3}(p) B^{\prime}\left(\bar{\chi}_{v}\right)\left(1+2 I_{2}(p) B^{\prime \prime}\left(\bar{\chi}_{0}\right)\right) H_{0}(p)\right] \\
& +\frac{T^{2}}{N}\left[-\frac{1}{2} \int_{p} \log \left[1-4 I_{2}(p) B^{\prime \prime}\left(\bar{\chi}_{v}\right)\right]\right. \\
& +2 \int_{p} I_{2}(p) H_{v}(p)\left[1+2 I_{2}(p) B^{\prime \prime}\left(\bar{\chi}_{0}\right)\right]\left[1-4 I_{2}(p) B^{\prime \prime}\left(\bar{\chi}_{0}\right)\right] \\
& +2 \int_{p} I_{2}(p)^{2} H_{v}(p)^{2}\left[1-4 I_{2}(p) B^{\prime \prime}\left(\bar{\chi}_{0}\right)\right]^{2} \\
& \left.-16 I_{2} \int_{p} I_{2}(p) B^{\prime}\left(\bar{\chi}_{v}\right)\left[1+6 I_{2}(p) H_{0}(p)\right] B^{\prime \prime \prime}\left(\bar{\chi}_{0}\right)\right] \\
& H_{v}(p)=\frac{B^{\prime \prime}\left(\bar{\chi}_{v}\right)}{1-4 I_{2}(p) B^{\prime \prime}\left(\bar{\chi}_{v}\right)},
\end{aligned}
$$

where $v^{2}$ stands for $v_{a b}^{2}$ and is the argument of the function. The integrals $I_{n}$ and $I_{n}(q)$ are defined in appendix I and we recall that:

$$
\bar{\chi}_{v}=v^{2}+2 T I_{1}+4 I_{2}\left(B^{\prime}\left(\bar{\chi}_{v}\right)-B^{\prime}\left(\bar{\chi}_{0}\right)\right)
$$

\subsection{The effective action in terms of the renormalized disorder}

To make the conversion to renormalized disorder, we use that on the r.h.s. of (6.2) we can express $B$ in terms of the renormalized $\tilde{B}$ at leading order. Using (3.8)

$$
\tilde{B}^{\prime}(x)=B^{\prime}\left(x+2 T I_{1}+4 I_{2}\left(\tilde{B}^{\prime}(x)-\tilde{B}^{\prime}(0)\right)\right)
$$




$$
x:=v^{2}
$$

and differentiating (6.5) w.r.t $x$, we obtain

$$
\frac{\tilde{B}^{\prime \prime}(x)}{1+4 I_{2} \tilde{B}^{\prime \prime}(x)}=B^{\prime \prime}\left(x+2 T I_{1}+4 I_{2}\left(\tilde{B}^{\prime}(x)-\tilde{B}^{\prime}(0)\right)\right)
$$

This allows to rewrite (6.3) as (already noted in (4.25))

$$
\begin{aligned}
& \tilde{H}_{x}(p):=\frac{\tilde{B}^{\prime \prime}(x)}{1+4\left(I_{2}-I_{2}(p)\right) \tilde{B}^{\prime \prime}(x)}=H_{v}(p) \\
& \delta \tilde{B}(x)=\frac{1}{N}\left\{8 \int_{p} C(p)^{2}\left[\tilde{H}_{x}(p) x\right]^{2}\right. \\
& +64 \int_{p} I_{3}(p) C(p) \tilde{H}_{x}(p)^{2} x\left[\tilde{B}^{\prime}(x)-\tilde{B}^{\prime}(0)\right] \\
& +128 \int_{p} I_{3}(p)^{2} \tilde{H}_{x}(p)^{2}\left[\tilde{B}^{\prime}(x)-\tilde{B}^{\prime}(0)\right]^{2} \\
& +8 \int_{p} C(p)^{2} x \tilde{H}_{x}(p)\left[\tilde{B}^{\prime}(x)-\tilde{B}^{\prime}(0)\right] \\
& \left.+16 \int_{p} \tilde{H}_{x}(p)\left[\tilde{B}^{\prime}(x)-\tilde{B}^{\prime}(0)\right]^{2} I_{4}(p)\right\} \\
& +\frac{T}{N}\left\{4 \int_{p}\left[C(p) x+4 I_{3}(p)\left(\tilde{B}^{\prime}(x)-\tilde{B}^{\prime}(0)\right)\right] \tilde{H}_{x}(p)\left[1+2 I_{2}(p) \tilde{H}_{x}(p)\right]\right. \\
& -32 \int_{p} I_{2}(p)^{2}\left[C(p) x+4 I_{3}(p)\left(\tilde{B}^{\prime}(x)-\tilde{B}^{\prime}(0)\right)\right] \tilde{H}_{x}(p)^{2} \frac{\tilde{B}^{\prime \prime}(0)}{1+4 I_{2} \tilde{B}^{\prime \prime}(0)} \\
& \left.-16 \int_{p} I_{3}(p) \tilde{B}^{\prime}(x)\left(1+2 I_{2}(p) \frac{\tilde{B}^{\prime \prime}(0)}{1+4 I_{2} \tilde{B}^{\prime \prime}(0)}\right) \tilde{H}_{0}(p)\right\} \\
& +\frac{T^{2}}{N}\left\{-\frac{1}{2} \int_{p} \ln \left(\frac{1+4 \tilde{B}^{\prime \prime}(x)\left[I_{2}-I_{2}(p)\right]}{1+4 \tilde{B}^{\prime \prime}(x) I_{2}}\right)\right. \\
& +2 \int_{p} I_{2}(p) \tilde{H}_{x}(p)\left[1+2 I_{2}(p) \frac{\tilde{B}^{\prime \prime}(0)}{1+4 I_{2} \tilde{B}^{\prime \prime}(0)}\right]\left[1-4 I_{2}(p) \frac{\tilde{B}^{\prime \prime}(0)}{1+4 I_{2} \tilde{B}^{\prime \prime}(0)}\right] \\
& +2 \int_{p} I_{2}(p)^{2} \tilde{H}_{x}(p)^{2}\left[1-4 I_{2}(p) \frac{\tilde{B}^{\prime \prime}(0)}{1+4 I_{2} \tilde{B}^{\prime \prime}(0)}\right]^{2} \\
& \left.-16 I_{2} \int_{p} I_{2}(p) \tilde{B}^{\prime}(x)\left[1+6 I_{2}(p) \tilde{H}_{0}(p)\right] \frac{\tilde{B}^{\prime \prime \prime}(0)}{\left(1+4 I_{2} \tilde{B}^{\prime \prime}(0)\right)^{3}}\right\}
\end{aligned}
$$

\subsection{Expression in terms of rescaled dimensionless quantities}

As in Ref. [6], we define the dimensionless function $b$ of the dimensionless argument $z$ through

$$
b(z)=4 A_{d} m^{4 \zeta-\epsilon} \tilde{B}\left(z m^{-2 \zeta}\right) .
$$


where $\epsilon=4-d$ and $A_{d}=2(4 \pi)^{-d / 2} \Gamma\left(3-\frac{d}{2}\right)$. This prefactor has been chosen to simplify the leading-order $\beta$-function. For $d<4$ and $T=0$ all integrals which will appear here and below are convergent in the limit of UV cutoff $\Lambda \rightarrow \infty$ that we consider, e.g. in that limit $I_{2}=A_{d} m^{-\epsilon} / \epsilon$. The rescaling factor $\zeta$ is for now unspecified, but at the fixed point it will yield the roughness exponent.

In terms of rescaled quantities, one finds that the result of the $1 / N$ expansion for the second cumulant of the disorder can be rewritten as

$$
b_{m}(x)=b_{m}^{0}(x)+\frac{1}{N} b^{1}\left[b_{m}^{0}\right](x),
$$

where $b_{m}^{0}(x)$ is the solution to dominant order, obtained previously for an arbitrary bare disorder. We have made apparent the dependence on the mass $m$ (and raise the indices whenever necessary). The expression for the $O(1 / N)$ correction $b_{1}[b](x)$ can be obtained from the last subsection upon rescaling and reads (for $d<4)$ :

$$
\begin{aligned}
& b_{1}[b](x)=\frac{1}{A_{d}} \int_{p}\left\{2 c(p)^{2}\left[h_{x}(p) x\right]^{2}+4 i_{3}(p) c(p) h_{x}(p)^{2} x\left[b^{\prime}(x)-b^{\prime}(0)\right]\right. \\
& +2 i_{3}(p)^{2} h_{x}(p)^{2}\left[b^{\prime}(x)-b^{\prime}(0)\right]^{2}+2 c(p)^{2} x h_{x}(p)\left[b^{\prime}(x)-b^{\prime}(0)\right] \\
& \left.+h_{x}(p)\left[b^{\prime}(x)-b^{\prime}(0)\right]^{2} i_{4}(p)\right\} \\
& +\frac{\epsilon T_{m}}{A_{d}} \int_{p}\left\{\left[c(p) x+i_{3}(p)\left(b^{\prime}(x)-b^{\prime}(0)\right)\right] h_{x}(p)\left[1+\frac{1}{2} i_{2}(p) h_{x}(p)\right]\right. \\
& -\frac{1}{2} i_{2}(p)^{2}\left[c(p) x+i_{3}(p)\left(b^{\prime}(x)-b^{\prime}(0)\right)\right] h_{x}(p)^{2} \frac{b^{\prime \prime}(0)}{1+i_{2} b^{\prime \prime}(0)} \\
& \left.-i_{3}(p) b^{\prime}(x)\left(1+\frac{1}{2} i_{2}(p) \frac{b^{\prime \prime}(0)}{1+i_{2} b^{\prime \prime}(0)}\right) h_{0}(p)\right\} \\
& +\frac{\epsilon^{2} T_{m}^{2}}{4 A_{d}} \int_{p}\left\{-\frac{1}{2} \ln \left(\frac{1+b^{\prime \prime}(x)\left[i_{2}-i_{2}(p)\right]}{1+b^{\prime \prime}(x) i_{2}}\right)\right. \\
& +\frac{1}{2} i_{2}(p) h_{x}(p)\left[1+\frac{1}{2} i_{2}(p) \frac{b^{\prime \prime}(0)}{1+i_{2} b^{\prime \prime}(0)}\right]\left[1-i_{2}(p) \frac{b^{\prime \prime}(0)}{1+i_{2} b^{\prime \prime}(0)}\right] \\
& +\frac{1}{8} i_{2}(p)^{2} h_{x}(p)^{2}\left[1-i_{2}(p) \frac{b^{\prime \prime}(0)}{1+i_{2} b^{\prime \prime}(0)}\right]^{2} \\
& \left.-i_{2} i_{2}(p) b^{\prime}(x)\left[1+\frac{3}{2} i_{2}(p) h_{0}(p)\right] \frac{b^{\prime \prime \prime}(0)}{\left(1+i_{2} b^{\prime \prime}(0)\right)^{3}}\right\}
\end{aligned}
$$

where one has defined

$$
h_{x}(p)=\frac{b^{\prime \prime}(x)}{1+\left[i_{2}-i_{2}(p)\right] b^{\prime \prime}(x)}
$$

as well as the rescaled temperature:

$$
T_{m}:=\frac{4 A_{d} m^{\theta}}{\epsilon} T
$$

where $\theta=d-2+2 \zeta$ is the energy fluctuation exponent, and the rescaled integrals are denoted by small letters:

$$
i_{n}(p):=\left.\frac{1}{A_{d}} I_{n}(p)\right|_{m=1} \equiv \frac{1}{A_{d}} \int \frac{\mathrm{d}^{d} p}{(2 \pi)^{d}} \ldots
$$




$$
c(p):=\frac{1}{1+p^{2}}
$$

with, e.g $i_{2}=i_{2}(p=0)=1 / \epsilon$. In the expression above we have kept the order of the diagrams from section $4.3 \mathrm{ff}$. . Note that explicit $\Lambda$ dependence can be reinstated in (6.12) by restricting all rescaled momentum integrals by $\Lambda / m$ as upper cutoff, and is necessary in $T>0$ integrals (since they are usually UV divergent).

Regrouping terms, this result can be rewritten in a more compact form:

$$
\begin{aligned}
b_{1}(x)= & 2 x^{2} g_{1}\left(a_{x}\right)+2 x\left(b^{\prime}(x)-b^{\prime}(0)\right) g_{2}\left(a_{x}\right)+2\left(b^{\prime}(x)-b^{\prime}(0)\right)^{2} g_{3}\left(a_{x}\right) \\
& +\epsilon T_{m}\left[x\left(g_{4}\left(a_{x}\right)+a_{0} g_{5}\left(a_{x}\right)\right)+\left(b^{\prime}(x)-b^{\prime}(0)\right)\left(g_{6}\left(a_{x}\right)+a_{0} g_{7}\left(a_{x}\right)\right)\right. \\
& \quad+b^{\prime}(x)\left(\left(g_{8}\left(a_{0}\right)+a_{0} g_{9}\left(a_{0}\right)\right)\right] \\
& +\left(\epsilon T_{m}\right)^{2}\left[g_{10}\left(a_{x}\right)+a_{0} g_{11}\left(a_{x}\right)+a_{0}^{2} g_{12}\left(a_{x}\right)+b^{\prime}(x) \alpha\left(\gamma+a_{0} g_{13}\left(a_{0}\right)\right)\right]
\end{aligned}
$$

with

$$
a_{x}=\frac{b^{\prime \prime}(x)}{1+\frac{b^{\prime \prime}(x)}{\epsilon}}, \quad h_{x}(p)=\frac{a_{x}}{1-a_{x} i_{2}(p)}, \quad \alpha=\frac{b^{\prime \prime \prime}(0)}{\left(1+\frac{b^{\prime \prime}(0)}{\epsilon}\right)^{3}}, \quad \bar{\alpha}=\frac{b^{\prime \prime \prime \prime}(0) / \epsilon}{\left[1+b^{\prime \prime}(0) / \epsilon\right]^{2}}-\frac{2 b^{\prime \prime \prime}(0)^{2} / \epsilon^{2}}{\left[1+b^{\prime \prime}(0) / \epsilon\right]^{3}}
$$

and

$$
\begin{aligned}
g_{1}\left(a_{x}\right) & =\frac{1}{A_{d}} \int_{p} c(p)^{2} h_{x}(p)^{2} \\
g_{2}\left(a_{x}\right) & =\frac{1}{A_{d}} \int_{p}\left[2 c(p) i_{3}(p) h_{x}(p)^{2}+c(p)^{2} h_{x}(p)\right] \\
g_{3}\left(a_{x}\right) & =\frac{1}{A_{d}} \int_{p}\left[\frac{1}{2} i_{4}(p) h_{x}(p)+i_{3}(p)^{2} h_{x}(p)^{2}\right] .
\end{aligned}
$$

The other functions $g_{i}(a)$ and $\gamma$, which characterize non-zero temperature, are given in Appendix $\mathrm{H}$.

Finally, note that the results (6.12) and (6.17) given above are for the choice $\zeta=0$. For a non-zero $\zeta=\zeta_{0}+\frac{1}{N} \zeta_{1}+\ldots$ the result for $b_{1}[b](x)$ is identical to (6.12), (6.17) above, up to the trivial linear rescaling term

$$
b_{1}[b](x) \rightarrow b_{1}[b](x)-\left(2 \zeta_{1} x b^{\prime}(x)+4 \zeta_{1} b(x)\right) \ln (m)
$$

\section{The $\beta$-function at order $1 / N$}

We are now ready to obtain the flow equation of the dimensionless disorder, i.e. compute the $\beta$-function. Here we give the most direct method to do so and give the results. A second method, closer in spirit to the diagrammatic approach and which yields more compact expressions has also been devised. Since it is rather involved it is detailed in appendix G.2.

Our goal in the present paper is as follows. The dimensionless disorder $b(x)$ depends on the IR cutoff $m$, and a priori, also on the UV cutoff $\Lambda$. To obtain a FRG flow equation we want to express:

$$
-m \partial_{m} b(x)=\beta[b](x)=\beta_{0}[b](x)+\frac{1}{N} \beta_{1}[b](x)+\cdots
$$

in terms of $b(x)$, at fixed $\Lambda$. Furthermore, we are interested in the behavior of the resulting expression when $m / \Lambda$ becomes very small, which we hope can be made independent of $\Lambda / m$, if necessary with appropriate redefinitions of parameters. 
To compute $\beta[b](x)$ in the $1 / N$ expansion we write

$$
b(x)=b_{0}(x)+\frac{1}{N} b_{1}\left[b_{0}\right](x)+O\left(\frac{1}{N^{2}}\right),
$$

where $b_{0}(x)$ is the dimensionless disorder at leading order. The corresponding $\beta$-function was derived in Ref. [6]. It can be recovered by inserting (6.10) into (3.14) using $m \partial_{m} I_{1}=-2 m^{2} I_{2}$ and the above value of $I_{2}$ (for $\Lambda=\infty$ ). In the variables $b$ it reads

$$
\begin{aligned}
-m \partial_{m} b_{0}(x) & =\beta_{0}\left[b_{0}\right](x) \\
\beta_{0}[b](x) & =(\epsilon-4 \zeta) b(x)+2 \zeta x b^{\prime}(x)+\frac{1}{2} b^{\prime}(x)^{2}-b^{\prime}(x) b^{\prime}(0)+T_{m} \frac{b^{\prime}(x)}{1+\frac{b^{\prime \prime}(0)}{\epsilon}},
\end{aligned}
$$

where we recall $T_{m}=4 T A_{d} m^{\theta} / \epsilon$. Thus the $\beta$-function at leading order has a simple and well-defined $\Lambda=\infty$ limit.

We now turn to the next order correction.

\subsection{The $\beta$-function at $T=0$}

We first detail the $T=0$ limit, which has a well-defined $\Lambda=\infty$ limit. At order $1 / N$, deriving (7.2) w.r.t. $m$, we obtain

$$
-m \partial_{m} b=\beta_{0}\left[b_{0}\right]+\frac{1}{N} \beta_{0}\left[b_{0}\right] \frac{\mathrm{d} b_{1}\left[b_{0}\right]}{\mathrm{d} b_{0}}+O\left(\frac{1}{N^{2}}\right) .
$$

Replacing $b_{0}$ by $b$, using (7.2), we obtain the $\beta$-function at order $1 / N$

$$
\begin{aligned}
\beta[b] & =\beta_{0}\left[b-\frac{1}{N} b_{1}[b]\right]+\frac{1}{N} \beta_{0}[b] \frac{\mathrm{d} b_{1}[b]}{\mathrm{d} b}+O\left(\frac{1}{N^{2}}\right) \\
& =\beta_{0}[b]+\frac{1}{N}\left\{\beta_{0}[b] \frac{\mathrm{d} b_{1}[b]}{\mathrm{d} b}-b_{1}[b] \frac{\mathrm{d} \beta_{0}[b]}{\mathrm{d} b}\right\}+O\left(\frac{1}{N^{2}}\right) .
\end{aligned}
$$

This expression is still symbolic. The derivative $\frac{\mathrm{d}}{\mathrm{d} b} b_{1}[b]$ e.g. is the sum over the derivatives w.r.t. all derivatives of $b$, and in the above expression is multiplied by the $\beta$-function of the corresponding derivative, obtained by deriving (7.3) w.r.t. $x$. Since at $T=0, b_{1}[b](x)$ depends only on $b^{\prime}(x)-b^{\prime}(0)$ and $b^{\prime \prime}(x),(7.5)$ gives:

$$
\begin{aligned}
\beta[b]= & \frac{\delta b_{1}[b](x)}{\delta b^{\prime \prime}[x]} \beta_{0}[b]^{\prime \prime}(x)+\frac{\delta b_{1}[b](x)}{\delta\left(b^{\prime}[x]-b^{\prime}(0)\right)}\left(\beta_{0}[b]^{\prime}(x)-\beta_{0}[b]^{\prime}(0)\right) \\
& -\left\{\epsilon b_{1}[b](x)+b_{1}[b]^{\prime}(x)\left[b^{\prime}(x)-b^{\prime}(0)\right]-b^{\prime}(x) b_{1}[b]^{\prime}(0)\right\}
\end{aligned}
$$

Inserting the expression (6.12) with $T=0$ one finds:

$$
\begin{aligned}
\beta(b(x))= & \epsilon b(x)+\frac{1}{2} b^{\prime}(x)^{2}-b^{\prime}(x) b^{\prime}(0)+\frac{1}{N} \frac{1}{A_{d}} \int \frac{\mathrm{d}^{d} p}{(2 \pi)^{d}} \\
& \left\{2 x c(p)^{2} h_{x}(p)^{2}\left[x \epsilon+2 x \epsilon h_{x}(p) i_{2}(p)+2 b^{\prime}(0)-2 b^{\prime}(x)\right]\right. \\
& +4 c(p) h_{x}(p)^{2} i_{3}(p)\left[b^{\prime}(x)-b^{\prime}(0)\right]\left[2 x \epsilon+2 x \epsilon h_{x}(p) i_{2}(p)+b^{\prime}(0)-b^{\prime}(x)\right] \\
& +2 \epsilon h_{x}(p)^{2}\left[3+2 h_{x}(p) i_{2}(p)\right] i_{3}(p)^{2}\left[b^{\prime}(0)-b^{\prime}(x)\right]^{2} \\
& +2 c(p)^{2} h_{x}(p)\left[b^{\prime}(x)-b^{\prime}(0)\right]\left[x \epsilon\left(1+i_{2}(p) h_{x}(p)\right)+b^{\prime}(0)-b^{\prime}(x)\right] \\
& \left.+\epsilon h_{x}(p)\left[2+h_{x}(p) i_{2}(p)\right] i_{4}(p)\left[b^{\prime}(0)-b^{\prime}(x)\right]^{2}\right\} .
\end{aligned}
$$


A more compact expression can be found if one uses (6.17) as a starting point. The first term on the 1.h.s. of (7.6) is replaced by $\frac{\delta b_{1}}{\delta a_{x}}\left(-m \partial_{m}^{0} a_{x}\right)$ and one uses

$$
-m \partial_{m}^{0} a_{x}=\epsilon a_{x}+\left[b^{\prime}(x)-b^{\prime}(0)\right] b^{\prime \prime \prime}(x)\left(a_{x}\right)^{2}=\epsilon a_{x}+\left[b^{\prime}(x)-b^{\prime}(0)\right] \partial_{x} a_{x} .
$$

One finds a form rather similar to (6.17):

$$
\begin{aligned}
-m \partial_{m} b= & \epsilon b+\frac{1}{2} b^{\prime 2}-b^{\prime} b^{\prime}(0) \\
& +\frac{1}{N}\left[2 x^{2} \tilde{g}_{1}(a)+2 x\left(b^{\prime}(x)-b^{\prime}(0)\right) \tilde{g}_{2}(a)+2\left(b^{\prime}(x)-b^{\prime}(0)\right)^{2} \tilde{g}_{3}(a)\right]
\end{aligned}
$$

with

$$
\begin{aligned}
& \tilde{g}_{1}(a)=\epsilon\left(a g_{1}^{\prime}(a)-g_{1}(a)\right) \\
& \tilde{g}_{2}(a)=\epsilon a g_{2}^{\prime}(a)-2 g_{1}(a) \\
& \tilde{g}_{3}(a)=\epsilon\left(g_{3}(a)+a g_{3}^{\prime}(a)\right)-g_{2}(a) .
\end{aligned}
$$

All terms proportional to $\partial_{x} a_{x}$ have canceled. Also note that we have used analyticity in the derivation. Issues related to the non-analytic regime will be discussed in a subsequent publication.

\subsection{The $\beta$-function at non-zero temperature}

At non-zero temperature the $\beta$-function to order $1 / N$ is independent of the UV cutoff $\Lambda$ only for $d<2$. Its expression is more complicated and we give here only its form, for $d<2$. The derivation and explicit expressions for the functions are given in Appendix $\mathrm{H}$, together with some comments about $d>2$.

$$
\begin{aligned}
\beta_{1}[b](x)= & \beta_{1}^{T=0}[b](x)+T_{m}\left[x\left(\tilde{g}_{4}\left(a_{x}\right)+a_{0} \tilde{g}_{5}\left(a_{x}\right)\right)+b^{\prime}(x)\left(\tilde{g}_{6}\left(a_{x}\right)+a_{0} \tilde{g}_{7}\left(a_{x}\right)+\tilde{g}_{8}\left(a_{0}\right)\right)\right] \\
& +T_{m}^{2}\left[\tilde{g}_{10}\left(a_{x}\right)+a_{0} \tilde{g}_{11}\left(a_{x}\right)+a_{0}^{2} \tilde{g}_{12}\left(a_{x}\right)+x a_{0}^{\prime}\left(\epsilon-a_{0}\right) g_{5}\left(a_{x}\right)+\alpha b^{\prime}(x)\left(\epsilon g_{7}\left(a_{x}\right)+\phi\left(a_{0}\right)\right)\right] \\
& +T_{m}^{3}\left[b^{\prime}(x)\left(\psi\left(a_{0}\right) \alpha^{2}+\tilde{\psi}\left(a_{0}\right) \bar{\alpha}\right)+\alpha \epsilon^{2}\left(g_{11}\left(a_{x}\right)+2 a_{0} g_{12}\left(a_{x}\right)\right)\right]
\end{aligned}
$$

where the $T=0$ expression $\beta_{1}^{T=0}[b](x)$ was given above.

\section{Conclusion}

In this article we have computed the effective action of the field theory of random manifolds at large $N$. The 2-replica part of this quantity is what is needed to compute the renormalized disorder to order $1 / N$. Although similar in spirit (one must compute the determinant of fluctuations around a $N=\infty$ saddle point) the problem solved here is much more complex than for the standard $1 / N$ expansion (say in the $\phi^{4}$-model), first because one needs to perform the calculation of fluctuations around the saddle point at fixed averaged field value, second because this involves four-replica matrices. It does however not involve spontaneous replica-symmetry breaking of the Parisi type, but rather some type of simpler explicit (vector) symmetry breaking.

To handle such additional difficulties we have introduced in this paper two complementary methods. The first one is graphical and uses a diagrammatics which is able to handle both the $O(N)$ and the replica indices. In this diagrammatics the zero-temperature diagrams are reasonably easy to compute. Much more subtle are the finite- $T$ diagrams. Interestingly, only order $T$ and $T^{2}$ are found to be non-vanishing to this 
order in $1 / N$. It is even simpler for $d=0$, where the result for the $\beta$-function is polynomial. (This can be derived by using Bogoliubov's subtraction operator.) The second method uses an algebraic formula for the determinant of fluctuations around the saddle point. The algebra of the four-replica matrices is worked out and one uses an expansion in number of free replica sums to compute all components iteratively in a given order of this expansion. Since we only need the two-replica part, this is a finite calculation, although rather tedious and has to be performed with Mathematica. The two methods are complementary, since any forgotten diagram of the graphical method can be traced to some term in the algebraic result, and vice versa. They are also, to our knowledge, new, henceforth the detailed exposition.

Having obtained the effective action, we rewrote it in terms of the dimensionless renormalized disorder. By varying with respect to the infrared cutoff, we obtained the $\beta$-function to order $1 / N$. We noted that this $\beta$-function is UV finite at $T=0$.

It is important to note that the derivation was made, strictly speaking, using an analytic action. This is familiar for $N=\infty$, where the same strategy was applied successfully: Although the derivation was done in the analytic regime, the $\beta$-function could then be continued to the non-analytic one. This was done via a careful analysis of the solution when it reaches the Larkin scale. A similar analysis will be performed in a forthcoming publication, together with a comparison to the two-loop result, and a detailed analysis of the physical consequences of the FRG flow derived here.

\section{Acknowledgements}

It is a pleasure to thank the KITP at UC Santa Barbara, where part of this work was done. K.W. greatly acknowledges financial support from the German National Science Foundation DFG through Heisenberg grant Wi1932/1-1.

\section{A The large- $N$ formalism for the effective action}

Before studying specific models, in the following sections, we first present schematically the framework of the large- $N$ calculations.

\section{A.1 General properties of the $1 / N$ expansion}

The general problem can be formulated as follows. We want to compute the effective action $\Gamma[u]$ defined as the Legendre transform of $\mathcal{W}[J]=\ln \mathcal{Z}[J]$ ( $J$ being the source field conjugated to $u$ ), in the case where the partition function can be written as:

$$
\mathcal{Z}[J]=\int \mathcal{D}[\psi] \mathrm{e}^{-N S[\psi, j]}
$$

where $j=J / \sqrt{N}$ is the rescaled source and $\psi$ is some auxiliary field (or a set of such fields). Here all space coordinates and indices are suppressed and integrals and sums implicit, in order to exhibit the structure more clearly.

The first step is to write $\mathcal{W}[J]$ in an $1 / N$ expansion using the standard saddle point method. One finds:

$$
\begin{aligned}
& \mathcal{W}[J]=N \tilde{W}[j] \\
& \tilde{W}[j]=W^{0}[j]+\frac{1}{N} W^{1}[j]+\frac{1}{N^{2}} W^{2}[j]+\ldots,
\end{aligned}
$$


where the $j$-dependent saddle-point value $\psi_{j}$ of the auxiliary field is solution of

$$
S_{\psi}^{\prime}\left[\psi_{j}, j\right]=0
$$

The expansion yields

$$
\begin{aligned}
& W^{0}[j]=-S\left[\psi_{j}, j\right] \\
& W^{1}[j]=-\frac{1}{2} \operatorname{tr} \ln S_{\psi \psi}^{\prime \prime}\left[\psi_{j}, j\right] \\
& W^{2}[j]=-\frac{1}{4 !} S_{a b c d}^{\prime \prime \prime \prime}\left[\psi_{j}, j\right]\left\langle\phi_{a} \phi_{b} \phi_{c} \phi_{d}\right\rangle_{\left(S^{\prime \prime}\right)^{-1}}+\frac{1}{3 !^{2} 2} S_{a b c}^{\prime \prime \prime}\left[\psi_{j}, j\right] S_{e f g}^{\prime \prime \prime}\left[\psi_{j}, j\right]\left\langle\phi_{a} \phi_{b} \phi_{c} \phi_{e} \phi_{f} \phi_{g}\right\rangle_{\left(S^{\prime \prime}\right)^{-1}}
\end{aligned}
$$

More generally, the $W^{n}(j)$ are obtained from the loop expansion of the field theory:

$$
\ln Z[j]=N W^{0}[j]+\ln \int \mathcal{D}[\phi] \exp \left(-\frac{1}{2} \phi S^{\prime \prime}\left[\psi_{j}, j\right] \phi-\sum_{p=3}^{\infty} \frac{N^{1-\frac{p}{2}}}{p !} \partial_{\psi_{a_{1}}} \ldots \partial_{\psi_{a_{p}}} S\left[\psi_{j}, j\right] \phi_{a_{1}} \ldots \phi_{a_{p}}\right)
$$

In these formula, the indices $a, b, \ldots$ summarize all spatial coordinates, indices etc. of the field.

The effective action is then defined as:

$$
\begin{aligned}
& \Gamma[u]=N \tilde{\Gamma}[v=u / \sqrt{N}] \\
& \tilde{\Gamma}[v]=v j_{v}-\tilde{W}\left[j_{v}\right], \quad v=\tilde{W}^{\prime}\left[j_{v}\right]
\end{aligned}
$$

The equation for $j_{v}$ can formally be inverted into an expansion in $1 / N$ using (A.3),

$$
j_{v}=j_{v}^{0}+\frac{1}{N} j_{v}^{1}+\ldots
$$

which yields in turn the expansion for the effective action:

$$
\tilde{\Gamma}[v]=\sum_{p=0}^{\infty} N^{-p} \tilde{\Gamma}^{p}[v]
$$

One finds that the leading order is simply the Legendre transform of $W^{0}[j]$,

$$
\tilde{\Gamma}^{0}[v]=v j_{v}^{0}-W^{0}\left[j_{v}^{0}\right], \quad v=\left(W^{0}\right)^{\prime}\left[j_{v}^{0}\right]
$$

where here and below $\left(W^{n}\right)^{\prime}[j]=\partial_{j} W^{n}[j]$. Since one has $\partial_{v} \tilde{\Gamma}^{0}[v]=j_{v}^{0}$, it satisfies the self-consistent equation

$$
\tilde{\Gamma}^{0}[v]=v \partial_{v} \tilde{\Gamma}^{0}[v]-W^{0}\left(\partial_{v} \tilde{\Gamma}^{0}[v]\right)
$$

and one has the usual relation between the second derivative matrices $\left(W^{0}\right)^{\prime \prime}\left[j_{v}^{0}\right]=\left[\frac{\delta \tilde{\Gamma}^{0}[v]}{\delta v \delta v}\right]^{-1}$.

In this paper we use the result for the next order:

$$
\tilde{\Gamma}^{1}[v]=-W^{1}\left[j_{v}^{0}\right]
$$


For completeness we give the two next orders:

$$
\begin{aligned}
\tilde{\Gamma}^{2}[v]= & -W^{2}\left[j_{v}^{0}\right]+\frac{1}{2}\left(W^{1}\right)^{\prime}\left[j_{v}^{0}\right]\left(\partial_{j}^{2}\left(W^{0}\right)^{\prime \prime}\left[j_{v}^{0}\right]^{-1}\left(W^{1}\right)^{\prime}\left[j_{v}^{0}\right]\right. \\
= & -W^{2}\left(j_{v}^{0}\right)+\frac{1}{2} \frac{\delta \tilde{\Gamma}^{1}}{\delta v}\left(\frac{\delta^{2} \tilde{\Gamma}^{0}}{\delta v \delta v}\right)^{-1} \frac{\delta \tilde{\Gamma}^{1}}{\delta v} \\
\tilde{\Gamma}^{3}[v]= & -W^{3}\left[j_{v}^{0}\right]+\left(W^{1}\right)^{\prime}\left[j_{v}^{0}\right]\left(W^{0}\right)^{\prime \prime}\left[j_{v}^{0}\right]^{-1}\left(W^{2}\right)^{\prime}\left[j_{v}^{0}\right] \\
& -\frac{1}{2}\left(W^{1}\right)^{\prime}\left[j_{v}^{0}\right]\left(W^{0}\right)^{\prime \prime}\left[j_{v}^{0}\right]^{-1}\left(W^{1}\right)^{\prime \prime}\left[j_{v}^{0}\right]\left(W^{0}\right)^{\prime \prime}\left[j_{v}^{0}\right]^{-1}\left(W^{0}\right)^{\prime \prime}\left[j_{v}^{0}\right]^{-1}\left(W^{1}\right)^{\prime}\left[j_{v}^{0}\right] \\
& +\frac{1}{6}\left(W^{0}\right)^{\prime \prime \prime}\left[j_{v}^{0}\right]\left(W^{0}\right)^{\prime \prime}\left[j_{v}^{0}\right]^{-3}\left(W^{1}\right)^{\prime}\left[j_{v}^{0}\right]^{3}
\end{aligned}
$$

where the following graphical rules allow to restore correctly all index contractions and spatial integrals implicit in the schematic notation above. Denote $-\Gamma^{n}$ by a square with $n$ inside and $W^{n}$ by a circle with a $n$ inside. One treats the circled 1,2,3 etc as vertices and considers all tree graphs. A line is a propagator $W_{0}^{\prime \prime}\left[j_{v}^{0}\right]^{-1}$ and is thus not a vertex (it is summed in a line). The sum of these numbers is just the order. The formula are equivalent to:

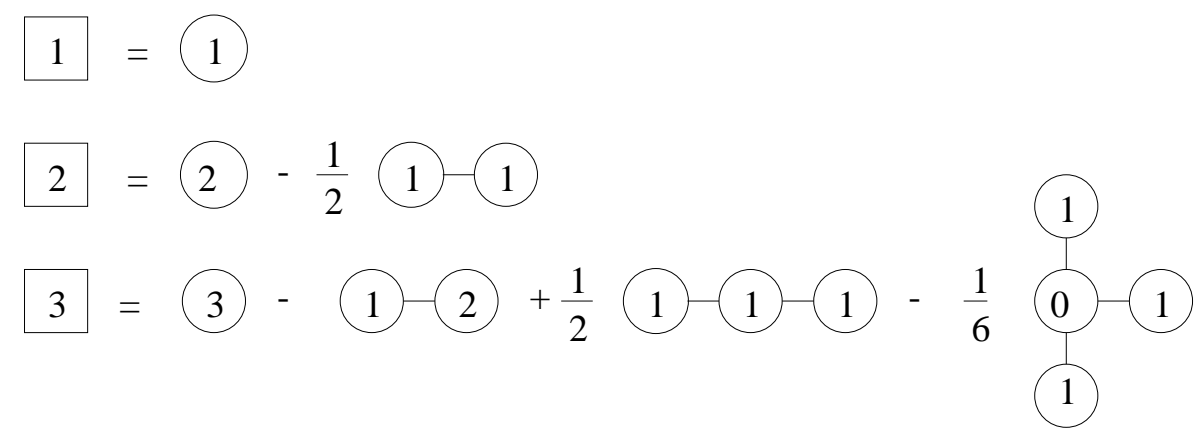

This has, including the combinatorial factors (from expanding the exponential and number of choices), an immediate interpretation as a perturbative expansion with all 1PI graphs subtracted. In the last line, the 1-2 graph comes with $1 / 2$ ! in expanding the exponential, and adding the two possibilities, the $1-1-1$ comes with $1 / 3$ !, but then there is a 3 for selecting the middle one, and the last graph has a $1 / 3$ ! from expanding the exponential. There is also a relative minus sign from each vertex added. To justify this graphical method, one simply recalls that quite generally correlation functions, i.e. $\mathcal{W}[J]$ are given as the sum of possible tree diagram made with $\Gamma[u]$ vertices. Since the Legendre transform is involutive, the same is true for $\Gamma[u]$ in terms of $\mathcal{W}[J]$ vertices. Thus to write $\tilde{\Gamma}^{n}[v]$ one must simply insert the proper orders in $1 / N$ at each vertex, in all possible ways, so as to match the total order.

Thus all the $\tilde{\Gamma}^{p}[v]$ can all be expressed as functions of the $W^{p}\left(j_{v}^{0}\right)$. Inserting the results (A.5), (A.6), a.s.o. from the saddle point expansion, one obtains the $\tilde{\Gamma}^{p}[v]$ explicitly in terms of derivatives of the $S$ functional in (A.1).

\section{A.2 Summary of main result}

Before we detail the calculations in a more pedagogical way for some specific models below, we first summarize here in compact notations the main result for the two lowest orders of the $1 / N$ expansion, with a generalization to a bilocal bare action.

Let us consider a $N$-component field theory whose action functional can be written as:

$$
S(\phi)=\frac{1}{2} \phi G^{-1} \phi+N S_{\mathrm{int}}\left[\psi_{x y}\right]
$$




$$
\psi_{x y}=\frac{1}{N} \phi_{x} \cdot \phi_{y}
$$

where $S_{\text {int }}$ is a functional of the bilocal field $\psi_{x y}$ (which is also a bi-index matrix, if the field $\phi$ carries other indices (e.g. $a, b, \ldots$ ). Then its effective action can be written as:

$$
\Gamma[\phi]=\frac{1}{2} \phi G^{-1} \phi+N \Gamma^{0}\left[\psi_{x y}\right]+\Gamma^{1}\left[\psi_{x y}\right]+\ldots,
$$

where $\Gamma^{0}$ is also a functional of a bilocal field and satisfies the self-consistent equation:

$$
\begin{aligned}
\frac{\delta \Gamma^{0}}{\delta \psi_{z t}}\left[\psi_{x y}\right] & =\frac{\delta S_{\mathrm{int}}}{\delta \psi_{z t}}\left[\psi_{x y}+G[\psi]_{x y}\right] \\
G[\psi]_{x y} & =\left\{G^{-1}+2 \frac{\delta \Gamma^{0}}{\delta \psi}[\psi]\right\}_{x y}^{-1}
\end{aligned}
$$

and

$$
\begin{aligned}
\Gamma^{1}\left[\psi_{x y}\right]=\frac{1}{2} \operatorname{tr} \ln \left[1_{z t, z^{\prime} t^{\prime}}+\right. & 2 \frac{\delta^{2} S_{i n t}}{\delta \psi_{z t} \delta \psi_{u v}}\left[\psi_{x y}+G[\psi]_{x y}\right] \times \\
& \left.\left(G[\psi]_{u t^{\prime}} G[\psi]_{v z^{\prime}}+\psi_{u t^{\prime}} G[\psi]_{v z^{\prime}}+\psi_{v z^{\prime}} G[\psi]_{u t^{\prime}}\right)\right],
\end{aligned}
$$

where $1_{x y a b, z t c d}=\delta_{x z} \delta_{y t} \delta_{a c} \delta_{b d}$.

\section{B Toy model}

For pedagogical clarity, we will give all details for the simpler case of the toy model.

\section{B.1 Model and effective action to leading order}

We study the following $O(N)$ toy model, defined by the partition sum:

$$
\begin{aligned}
\mathcal{Z}[J] & =\int \mathcal{D}[u] \mathrm{e}^{-S[u]+\sqrt{N} j u}=\int \mathcal{D}[u] \mathrm{e}^{-S[u, j]}=\int \mathcal{D}[u] \mathcal{D}[\chi] \mathcal{D}[\lambda] \mathrm{e}^{-S[u, \chi, \lambda, j]} \\
& =\int \mathcal{D}[\chi] \mathcal{D}[\lambda] \mathrm{e}^{-N S[\chi, \lambda, j]} \\
S[u, j] & =\int_{x} \frac{1}{2}(\nabla u)^{2}+\frac{1}{2} m^{2} u^{2}+N V\left(\frac{u^{2}}{N}\right)-\sqrt{N} j u \\
S[u, \chi, \lambda, j] & =S(u)-\int_{x}\left[\frac{1}{2} \lambda\left(N \chi-u^{2}\right)+\sqrt{N} j u\right] \\
S[\chi, \lambda, j] & =\frac{1}{2} \operatorname{tr} \ln \left(-\nabla^{2}+m^{2}+\lambda\right)+\int_{x}\left[V(\chi)-\frac{1}{2} \lambda \chi\right]-\frac{1}{2} \int_{x y} j_{x}\left(-\nabla^{2}+m^{2}+\lambda\right)_{x y}^{-1} j_{y},
\end{aligned}
$$

where $\lambda(x)$ and $\chi(x)$ are local fields (the factor $i$ has been absorbed in the field $\lambda$ ). The above expression (B.1) is thus of the form (A.1) where $\psi=(\chi, \lambda)$ is a set of two auxiliary fields.

The saddle point equation (A.4) thus read:

$$
\begin{aligned}
& \chi_{j}(x)=\left(-\nabla^{2}+m^{2}+\lambda_{j}\right)_{x x}^{-1}+j_{y^{\prime}}\left(-\nabla^{2}+m^{2}+\lambda_{j}\right)_{y^{\prime} x}^{-1}\left(-\nabla^{2}+m^{2}+\lambda_{j}\right)_{x y}^{-1} j_{y} \\
& \lambda_{j}(x)=2 V^{\prime}\left(\chi_{j}(x)\right) .
\end{aligned}
$$


From Appendix A one finds the dominant order:

$$
W^{0}[j]=-S\left[\chi_{j}, \lambda_{j}, j\right]
$$

Using the saddle point equation one finds:

$$
\left(W^{0}\right)^{\prime}[j]=-\partial_{j} S\left[\chi_{j}, \lambda_{j}, j\right]=\left(-\nabla^{2}+m^{2}+\lambda_{j}\right)^{-1} j
$$

Thus one obtains:

$$
\begin{aligned}
\Gamma(u) & =N \tilde{\Gamma}(u / \sqrt{N}), \quad v=u / \sqrt{N} \\
j_{v} & =\left(-\nabla^{2}+m^{2}+\lambda\right) v \\
\tilde{\Gamma}[v] & =j_{v} v+S\left[\chi_{j_{v}}, \lambda_{j_{v}}, j_{v}\right] \\
& =\frac{1}{2} v\left(-\nabla^{2}+m^{2}+\lambda\right) v+\frac{1}{2} \operatorname{tr} \ln \left(-\nabla^{2}+m^{2}+\lambda_{j_{v}}\right)+N V\left(\chi_{j_{v}}\right)-\frac{1}{2} \lambda_{j_{v}} \chi_{j_{v}} .
\end{aligned}
$$

One finds:

$$
\begin{aligned}
\tilde{\Gamma}(v) & =\frac{1}{2} \operatorname{tr} \ln G_{v}^{-1}+\int_{x} \frac{1}{2}(\nabla v)^{2}+\frac{1}{2} m^{2} v^{2}-\frac{1}{2}\left(\nabla^{2} G_{v}\right)_{x x}+V\left(v_{x}^{2}+\left(G_{v}\right)_{x x}\right) \\
\left(G_{v}^{-1}\right)_{x x^{\prime}} & =\left[-\nabla_{x}^{2}+m^{2}+2 V^{\prime}\left(v_{x}^{2}+\left(G_{v}\right)_{x x}\right)\right] \delta_{x x^{\prime}} .
\end{aligned}
$$

This can be simplified further as the complicated part of the effective action is just a constant. First one notes that $\tilde{\Gamma}(v)=\tilde{\Gamma}\left[G_{v}, v\right]$ satisfies:

$$
\left.\partial_{G} \tilde{\Gamma}[G, v]\right|_{G=G_{v}}=0
$$

As a consequence one sees that:

$$
\frac{\delta \tilde{\Gamma}[v]}{\delta v_{x}^{i}}=\left(-\nabla^{2}+m^{2}\right) v_{x}^{i}+2 v_{x}^{i} V^{\prime}\left(v_{x}^{2}+\left(G_{v}\right)_{x x}\right)
$$

The effective action (per unit volume $\Omega$ ) for a uniform configuration of the field $v_{x}=v$ reads:

$$
\begin{aligned}
\frac{1}{\Omega} \tilde{\Gamma}(v) & =\frac{1}{2} m^{2} v^{2}+V\left(v^{2}+G_{v}\right)+\frac{1}{2} \int_{q} \ln \left(q^{2}+m^{2}+2 V^{\prime}\left(v^{2}+G_{v}\right)\right)+\frac{q^{2}+m^{2}}{q^{2}+m^{2}+2 V^{\prime}\left(v^{2}+G_{v}\right)} \\
G_{v} & =\int_{q} \frac{1}{q^{2}+m^{2}+2 V^{\prime}\left(v^{2}+G_{v}\right)}
\end{aligned}
$$

which can also be written as

$$
\frac{1}{\Omega} \tilde{\Gamma}(v)=\frac{1}{2} m^{2} v^{2}+V\left(v^{2}+G_{v}\right)-G_{v} V^{\prime}\left(v^{2}+G_{v}\right)+\frac{1}{2} \int_{q} \ln \left(q^{2}+m^{2}+2 V^{\prime}\left(v^{2}+G_{v}\right)\right) .
$$

Here one can write:

$$
\begin{aligned}
\tilde{\Gamma}(v) & =\tilde{\Gamma}\left[G_{v}, v^{2}\right] \\
\left.\partial_{G} \tilde{\Gamma}\left[G, v^{2}\right]\right|_{G=G_{v}} & =0 .
\end{aligned}
$$

For uniform configurations the effective action is simply a function of $v^{2}$ such that

$$
\frac{1}{2} m^{2}+\tilde{V}^{\prime}\left(v^{2}\right)=\frac{1}{\Omega} \frac{\mathrm{d} \tilde{\Gamma}[v]}{\mathrm{d} v^{2}}=\frac{1}{2} m^{2}+V^{\prime}\left(v^{2}+G_{v}\right)
$$

i.e. it satisfies the self-consistent equation

$$
\tilde{V}^{\prime}\left(v^{2}\right)=V^{\prime}\left(v^{2}+\int_{q} \frac{1}{q^{2}+2 \tilde{V}^{\prime}\left(v^{2}\right)}\right)
$$

This defines a renormalized potential $\tilde{V}\left(v^{2}\right)$, whose RG flow is studied in Appendix I of [8]. 


\section{B.2 $1 / N$ corrections to the effective action}

To next order one has, from (A.6) :

$$
W^{1}[j]=-\frac{1}{2} \operatorname{tr} \ln S^{\prime \prime}\left[\chi_{j}, \lambda_{j}, j\right]
$$

where $\chi_{j}, \lambda_{j}$ are taken at their saddle point values (B.6). The symmetric matrix of second derivatives reads:

$$
\begin{aligned}
\left(S_{\chi \chi}^{\prime \prime}\right)_{x y} & =V^{\prime \prime}(\chi(x)) \delta_{x y} \quad, \quad\left(S_{\chi \lambda}^{\prime \prime}\right)_{x y}=-\frac{1}{2} \delta_{x y} \\
\left(S_{\lambda \lambda}^{\prime \prime}\right)_{x y} & =-\frac{1}{2} G_{x y} G_{y x}-(j G)_{x} G_{x y}(G j)_{y} \\
G_{x y} & =\left(-\nabla^{2}+m^{2}+\lambda\right)_{x y}^{-1} .
\end{aligned}
$$

It can be put in the form:

$$
S^{\prime \prime}=-\frac{1}{2}\left(\begin{array}{ll}
A & 1 \\
1 & B
\end{array}\right)
$$

Its inverse reads

$$
\begin{aligned}
\left(S^{\prime \prime}\right)^{-1} & =-2\left(\begin{array}{cc}
-B(1-A B)^{-1} & (1-B A)^{-1} \\
(1-A B)^{-1} & -A(1-B A)^{-1}
\end{array}\right) \\
A_{x y} & =-2 V^{\prime \prime}(\chi(x)) \delta_{x y} \\
B_{x y} & =G_{x y} G_{y x}+2\left(j_{v} G\right)_{x} G_{x y}(G j)_{y}=G_{x y} G_{y x}+2 v_{x} G_{x y} v_{y},
\end{aligned}
$$

where the last equality holds only for $j=j_{v}^{0}$. Note that $A$ and $B$ do not commute (unless fields are uniform).

The result for the effective action is thus, from (A.15)

$$
\begin{aligned}
\tilde{\Gamma}^{1}(v) & =\frac{1}{2} \operatorname{tr} \ln S^{\prime \prime}\left(\chi_{j_{v}^{0}}, \lambda_{j_{v}^{0}}, j_{v}^{0}\right) \\
j_{v}^{0} & =G^{-1} v \\
G_{x y} & =\left(-\nabla^{2}+m^{2}+\lambda_{j_{v}^{0}}\right)_{x y}^{-1}
\end{aligned}
$$

A more explicit form can be given for a uniform field configuration $v_{x}=v$, with in that case:

$$
\begin{aligned}
& A_{x y}=-2 V^{\prime \prime}(\chi) \delta_{x y}, \quad \chi=\chi_{j_{v}^{0}}=v^{2}+G_{x x} \\
& B_{x y}=\left(G_{v}^{x y}\right)^{2}+2 v^{2} G_{v}^{x y} \\
& \left(S^{\prime \prime}\right)^{-1}(q)=\frac{2}{1+2 V^{\prime \prime}\left(v^{2}+\int_{k} G_{v}(k)\right)\left[\Pi_{v}(q)+2 v^{2} G(q)\right]}\left(\begin{array}{cc}
\Pi_{v}(q)+2 v^{2} G(q) & -1 \\
-1 & -2 V^{\prime \prime}\left(v^{2}+\int_{k} G_{v}(k)\right)
\end{array}\right) \\
& \Pi_{v}(q)=\int_{k} G_{v}(q-k) G_{v}(k) \\
& G_{v}(k)=\frac{1}{k^{2}+m^{2}+2 V^{\prime}\left(v^{2}+\int_{k} G_{v}(k)\right)}
\end{aligned}
$$

yielding finally

$$
\tilde{\Gamma}^{1}(v)=\frac{1}{2} \int_{q} \ln (1-A(q) B(q))=\frac{1}{2} \int_{q} \ln \left(1+2 V^{\prime \prime}\left(v^{2}+\int_{k} G_{v}(k)\right)\left(\Pi_{v}(q)+2 v^{2} G_{q}(v)\right)\right)
$$

up to a constant. 


\section{Calculation of order $1 / N$ for the random manifold}

We now sketch the derivation of $\tilde{\Gamma}^{1}$ for the case of the random manifold.

It was shown in Ref. [8] that the partition sum (2.4) can be put in the form:

$$
\begin{aligned}
\mathcal{Z}[J]= & \int D \chi D \lambda e^{-N S[\chi, \lambda, j]} \\
S[\chi, \lambda, j]= & \frac{1}{2} \operatorname{tr} \ln \left(C^{-1}+i \lambda\right)+\int_{x} U(\chi(x))-\frac{i}{2} \lambda^{a b}(x) \chi^{a b}(x) \\
& -\frac{1}{2} \int_{x, x^{\prime}} j_{a}(x)\left(C^{-1}+i \lambda\right)_{a x, b x^{\prime}}^{-1} j_{b}\left(x^{\prime}\right),
\end{aligned}
$$

where the inversion and trace are performed in both replica space and spatial coordinate space. It has again the form (A.1) where $\psi=\left(\chi^{a b}(x), \lambda^{a b}(x)\right)$ is a set of 2-replica-matrix auxiliary fields. The saddle-point equation (A.4) reads (see Ref. [8]):

$$
\begin{aligned}
\chi_{j}^{a b}(x) & =\left(G_{j}\right)_{a x, b x}+\left(G_{j}: j\right)_{a x} \cdot\left(G_{j}: j\right)_{b x} \\
i \lambda_{j}^{a b}(x) & =2 \partial_{a b} U\left(\chi_{j}(x)\right) \\
G_{j}^{-1} & =C^{-1}+i \lambda_{j},
\end{aligned}
$$

where $G_{j}$ is a matrix with both replica indices and spatial coordinates and inversion is carried out for both. Here and below, replica indices are raised whenever explicit dependency is given, e.g. $\chi_{a b} \equiv \chi_{j}^{a b}$. The notation for the $N$-component vector $(G: j)_{b x}^{i}=\sum_{c} \int_{y} G_{b x, c y} j_{c}^{i}(y)$ is a shorthand for a matrix product, and everywhere we denote by

$$
\partial_{a b} U(\phi):=\partial_{\phi_{a b}} U(\phi)
$$

the simple derivative of the function $U(\phi)$ with respect to its matrix argument $\phi_{a b}$.

The dominant order, $W^{0}[j]$ and $\tilde{\Gamma}^{0}[v]$, was computed in Ref. [8], thus here we study only the next order. It is given by:

$$
\begin{aligned}
\tilde{\Gamma}^{1}[v] & =\frac{1}{2} \operatorname{tr} \ln S^{\prime \prime}\left[\chi_{j^{0}}, \lambda_{j^{0}}, j^{0}\right] \\
j_{a x}^{0} & =\left(G_{v}^{-1}: v\right)_{a x} \\
\left(G_{v}^{-1}\right)_{a x, b y} & =\left(C^{-1}\right)_{x, y} \delta_{a b}+2 \partial_{a b} U\left(\chi_{v}(x)\right) \delta^{d}(x-y) .
\end{aligned}
$$

Note that when computing the fluctuations around the saddle point we consider $\chi_{a b}$ and $\chi_{b a}$ as independently fluctuating fields, symmetry being restored at the saddle point (it is also possible to perform the calculation with symmetric matrices only).

The matrix of second derivatives can again be put in the form (see the previous section):

$$
S^{\prime \prime}=-\frac{1}{2}\left(\begin{array}{cc}
A & i 1 \\
i 1 & -B
\end{array}\right),
$$

and one can show that:

$$
\begin{aligned}
\operatorname{tr} \ln S^{\prime \prime} & =\operatorname{tr} \ln (1-A B) \\
A_{a b x, c d y} & =-2 \partial_{\chi_{a b}} \partial_{\chi_{c d}} U(\chi(x)) \delta_{x y} \\
B_{a b x, c d y} & =G_{a x, d y} G_{c y, b x}+(j: G)_{c y} G_{a x, d y}(G: j)_{b x}+(j: G)_{a x} G_{b x, c y}(G: j)_{d y}+M_{a b, c d} \\
& =G_{a x, d y} G_{c y, b x}+v_{b x} G_{a x, d y} v_{c y}+v_{a x} G_{b x, c y} v_{d y}+M_{a b, c d}
\end{aligned}
$$


and note that $A$ and $B$ do not commute (unless fields are uniform). The last equality is valid only when $j=j_{v}^{0}$ is inserted.

For uniform fields one finds:

$$
\begin{aligned}
\tilde{\Gamma}^{1}[v] & =\frac{1}{2} \int_{q} \ln \left(1-A(v) B_{q}(v)\right) \\
A(v)_{a b, c d} & =-2 \partial_{\chi_{a b}} \partial_{\chi_{c d}} U\left(\chi_{v}\right) \\
B_{q}(v)_{a b, c d} & =\int_{k} G_{v}^{a d}(k) G_{v}^{c b}(q-k)+v_{b} v_{c} G_{v}^{a d}(q)+v_{a} v_{d} G_{v}^{b c}(q) \\
G_{v}^{a b}(k) & =\left[C(k)^{-1}+i \lambda_{v}\right]_{a b}^{-1} \\
\chi_{v}^{c d} & =v_{c} v_{d}+\int_{k} G_{v}^{c d}(k) \\
i \lambda_{v}^{a b} & =2 \partial_{a b} U\left(\chi_{v}\right) .
\end{aligned}
$$

We note that the above matrices are not a priori symmetric in $a \leftrightarrow b$ or in $c \leftrightarrow d$ since we have chosen the representation where all components of the fields fluctuate freely. The alert reader will notice later (when coming back to the main text) that in fact the final matrix $A B_{q}$ will possess such a symmetry.

\section{The excluded replica-formalism}

In this section, we calculate the terms at order $T$ and $T^{2}$ using the excluded replica-formalism. This gives an independent derivation of the moon-shaped building blocks $\curvearrowright$ and $\curvearrowright$ in section 4.2.

We start by recalling that corrections at finite temperature are more difficult to obtain. The simplest example is given in the next appendix E. Here we want to understand this by making two contractions between $B\left(u_{a}(x)-u_{b}(x)\right)$ and $B\left(u_{a}(y)-u_{b}(y)\right)$. Be the first contraction (focusing on the replica-structure)

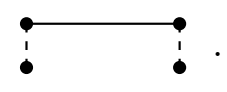

The following possible contractions are

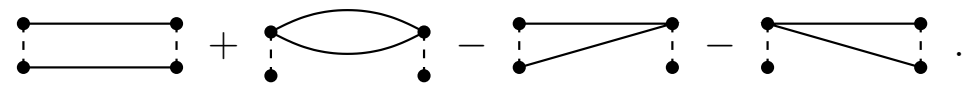

At zero temperature, we have found in section 4.3 that only the first term contributes. This enabled us to do the calculation at $T=0$.

The general case is more complicated, since there are now three more possible contractions, one may draw, i.e. 4 for each $B^{\prime \prime}$. This looks discouraging. Two ways out of the dilemma can be thought of. The first, called recursive construction or successive construction, tries to add one more link to a chain of $B^{\prime \prime}$. The decisive simplification then is that whenever arriving at e.g.

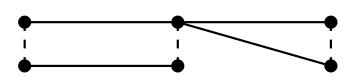

no more links can be added to the right, since the rightmost vertex is $B_{a a}^{\prime \prime}$, which is a constant. This procedure has however one crucial deficiency: Contractions are non-commutative! Let us illustrate this on the diagram

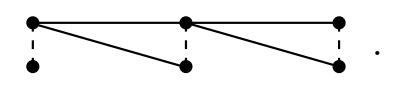

If one first does the two leftmost contractions, then the two rightmost contractions are not possible. On the other hand, when doing first the two rightmost contractions, the two leftmost are possible! Working 
with this formalism, i.e. using its implied simplifications, one has to number the lines. This is pretty awkward, since one wants to be able to interpret diagrams as such, without having to number lines. This is especially important here, since one wants to recognize the chains which give rise to $H_{a b}(p)$. Also note that problems arise at finite temperature, since there one has additional lines to add, and not all lines are needed to get a 2-replica term, i.e. there are lines which can be discarded, and still the diagram would be a 2-replica term. A very instructive example is the simplest 2-loop order diagram at finite temperature, which is derived in appendix E. One sees that diagrams can be grouped differently to cancel, and that these different cancellations correspond to different paths in the contractions.

One way out of the dilemma is the excluded replica approach, which at 2-loop order has as its descendent the sloop-algorithm [4]. Here we explain the excluded replica approach. We start by writing the trivial relation

$$
\sum_{a, b} B_{a b}=\sum_{a \neq b} B_{a b}+\sum_{a} B_{a a}
$$

The first term on the r.h.s. is the excluded replica disorder, the second a constant. When performing contractions, the second term does not contribute. Thus one can perform perturbation theory instead with $\sum_{a b} B_{a b}$ with $\sum_{a \neq b} B_{a b}$. The big advantage is that the last two terms in (D.2) do not contribute. The backdraw is that the final result is a sum over excluded replicas, which has to be projected onto the 2-replica term, and one may have more terms at intermediate steps. The final projection can be done formally, by replacing $B_{a b} \rightarrow B_{a b}\left(1-\delta_{a b}\right)$, expanding and then collecting the 2-replica contributions.

We now introduce the excluded replica-formalism. Recalling that

$$
\stackrel{p}{\longrightarrow}{ }_{b}^{a} \bigcirc{ }_{b}^{a}=\frac{1}{1-4 I_{2}(p) B^{\prime \prime}\left(\chi_{a b}\right)}{ }_{b}^{a}={ }_{b}^{a}=\frac{1}{2}\left[\left(u_{a}-u_{b}\right)^{2}\right] H_{a b}(p)\left[\left(u_{a}-u_{b}\right)^{2}\right] .
$$

We then proceed as follows: Whenever we have a long chain with some replica conserving lines (as above) and with doubled lines (or single lines when inserting vertices), we will always sum the double-line part, resulting in $H_{a b}$. We then have to sum explicitly the rest, and project it onto the 2-replica-contribution (in general). For illustration, we start with the open chain. This is, say when fixing one end to have replicas $a$ and $b$

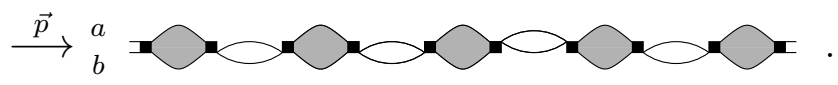

We furthermore observe that the combinatorial weight for either having a replica-conserving double line ( ), or a doubled line up ( $\bigcirc$ ) or down ( $)$ ) is the same. We do not draw crossed lines; by definition they are incorporated into the combinatorial factor. The above has to be projected onto the 2-replica sum. Writing any $H_{c d}(p)$ with excluded indices as $H_{c d}(p)\left(1-\delta_{c d}\right)$, and multiplying all terms, one always has to take the term with the $\delta_{c d}$, and (D.7), summed over all lengths of the chain, is

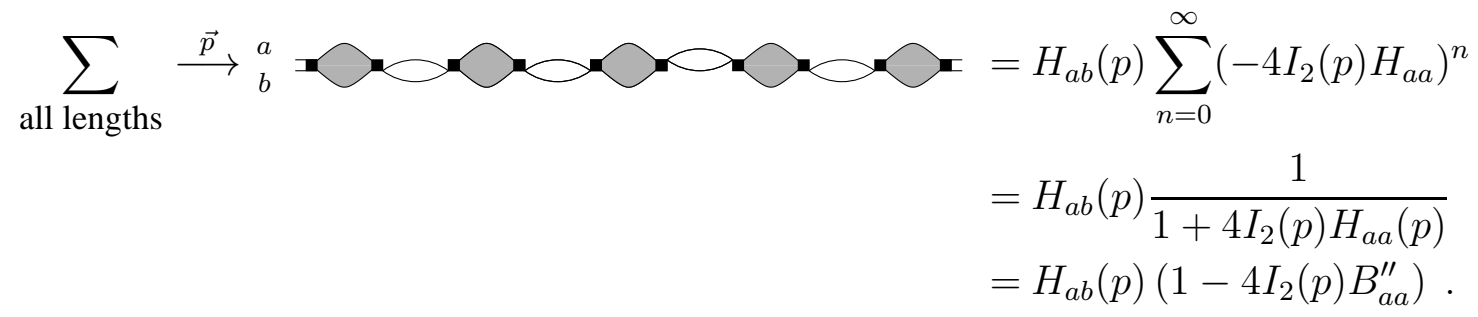

This object is well known: It is the chain times the moon-diagram, see table 5. We remark the important point that, even though we have an infinity of diagrams to sum up, in the projection onto 2 replicas (or since we are already fixing two replicas, maybe we should better say onto one replica) all but 1 non-trivial term 
vanish. (Also remark that we have been a bit sloppy to amputate the last $u^{2}$ legs. Otherwise, that is not so easy to represent.)

We need another intermediate result. Summing all diagrams which conserve the lower index, we obtain

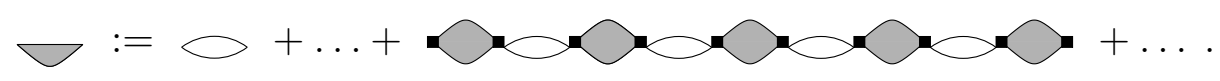

We want this sum $\square$, projected onto 1 replica, $\varpi_{1}$. Using the same procedure as above, we find

$$
\begin{aligned}
\mathcal{S}(p):=\bigvee_{1} & =2 I_{2}(p) \sum_{n=0}^{\infty}\left(-2 I_{2}(p) H_{a a}\right)^{n} \\
& =2 I_{2}(p) \frac{1}{1+2 I_{2}(p) H_{a a}} \\
& =2 I_{2}(p) \frac{1-4 I_{2}(p) B_{a a}^{\prime \prime}}{1-2 I_{2}(p) B_{a a}^{\prime \prime}} .
\end{aligned}
$$

The 2-replica contribution is

$$
\nabla_{2}=\mho_{1} \times \bigodot_{2} \times \mho_{1}=\mathcal{S}(p) H_{a b}(p) \mathcal{S}(p)
$$

This half-chain is very practical, since when inserted into a more complicated diagram, no further restrictions apply. In a diagram involving this half-chain and some "rest", there are two 2-replica contributions: The half-chain projected onto the 1-replica part times the rest projected onto 2 replicas (taking care of the replica-conserving line of the half-chain) or the 2-replica part of the half-chain times the 1-replica-part of the rest.

We continue on the order $T^{2}$-term. We have the following terms before projection:

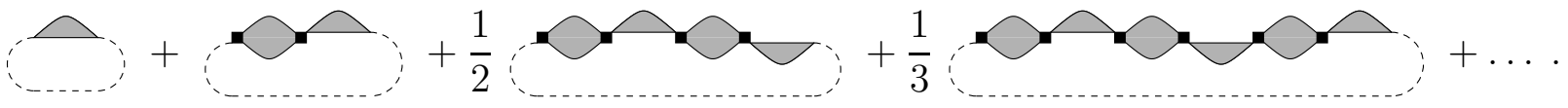

A dashed line denotes the identity; one would like to print this on a torus. The first term is very symbolic, since it is not a geometric sum but a log, and there should be at least one vertex. The second is 0 (coinciding replicas in the chain)

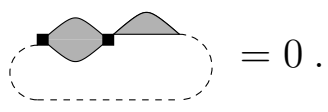

We now have the choice of where to project onto the 2-replica term. The rest has to be projected onto the 1-replica term. We first project one of the half-chains onto a 2-replica-term, using (D.11). The 2-replica term is shaded in dark grey. The rest consequently has to be projected onto the 1-replica terms, shaded in light grey. We obtain the class $\mathcal{C}_{1}$ :

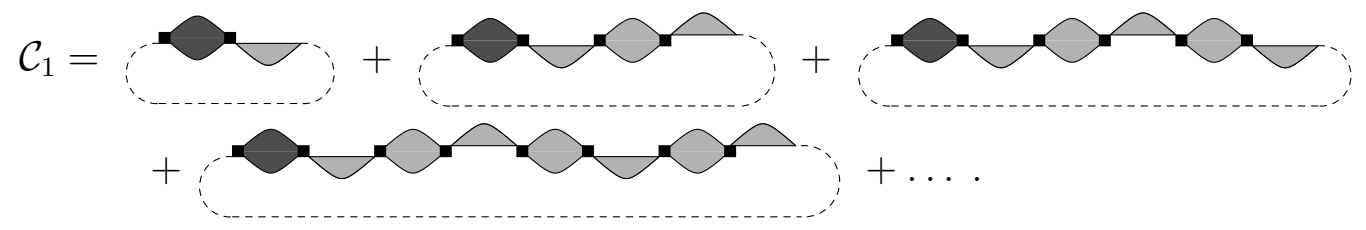

Denote by $n$ the number of $H_{a b}$ in each diagram, which have lines starting at different replicas. Each such $H_{a b}$ contributes a factor of $\left(1-\delta_{a b}\right)$, for a product of

$$
\sum_{i_{1}, i_{2}, i_{n}}\left(1-\delta_{i_{1} i_{2}}\right)\left(1-\delta_{i_{2} i_{3}}\right) \ldots\left(1-\delta_{i_{n} i_{1}}\right)
$$


We need all indices to be restricted to be identical. The terms which lead to that restriction are either the product of all $\delta$ 's ( 1 term) or any one of the $\delta$ 's left out. This gives another $n$ terms, but with a different sign, for a total of

$$
\mathcal{C}_{1}=\mathcal{S}(p) H_{a b}(p) \sum_{n=0}^{\infty}(1-n)\left[-H_{a a}(p) \mathcal{S}(p)\right]^{n},
$$

where it is important to note that also the first term in (D.14) for $n=0$ is correctly given by the above formula. We note the auxiliary sum

$$
\sum_{n=0}^{\infty}(1-n)(-x)^{n}=\frac{1+2 x}{(1+x)^{2}}
$$

We give the intermediate results

$$
\begin{aligned}
\frac{1}{1+H_{a a}(p) \mathcal{S}(p)} & =1-2 I_{2}(p) B_{a a}^{\prime \prime} \\
\frac{\mathcal{S}(p)}{1+H_{a a}(p) \mathcal{S}(p)} & =2 I_{2}(p)\left(1-4 I_{2}(p) B_{a a}^{\prime \prime}\right) \\
1+2 H_{a a}(p) \mathcal{S}(p) & =\frac{1+2 I_{2}(p) B_{a a}^{\prime \prime}}{1-2 I_{2}(p) B_{a a}^{\prime \prime}}
\end{aligned}
$$

the above sum is

$$
\mathcal{C}_{1}=2 I_{2}(p) H_{a b}(p)\left(1+2 I_{2}(p) B_{a a}^{\prime \prime}\right)\left(1-4 I_{2}(p) B_{a a}^{\prime \prime}\right) .
$$

This reproduces diagram (4.52).

We now turn to the diagrams where the half-chain is always projected onto the 1-replica contribution, whereas the non-trivial terms come from omitting a $\delta$ belonging to one of the $H_{a b}$ with lines entering into different replicas. With the same shading for 1- and 2-replica terms as above, this is

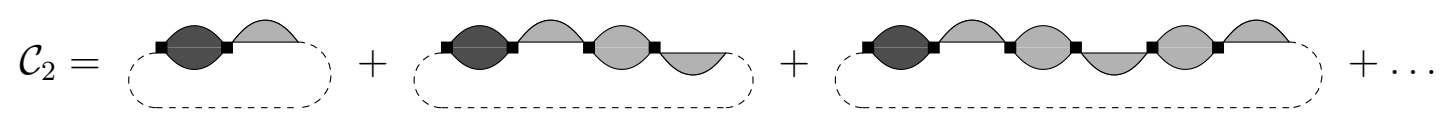

The first term is 0 , since it necessarily has crossed indices:

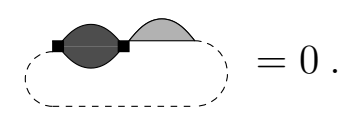

Each $H_{a b}$ which is projected onto a single replica again contributes a factor of $\left(1-\delta_{a b}\right)$ Starting from the second diagram, we have to leave out exactly $2 \delta$ 's in

$$
\frac{1}{n}\left(1-\delta_{i_{1} i_{2}}\right)\left(1-\delta_{i_{2} i_{3}}\right) \ldots\left(1-\delta_{i_{n} i_{1}}\right) .
$$

The first has already been left out in plotting (D.22), accounting for the factor of $n$. Leaving out two $\delta$ 's leads to a factor of $n(n-1) / 2$, since they are indistinguishable. The result contains two factors of $H_{a b}$; the remaining factors are all $H_{a a}$ :

$$
\mathcal{C}_{2}=\frac{1}{2} H_{a b}(p)^{2} \mathcal{S}(p)^{2} \sum_{n=2}^{\infty}(n-1)\left[-H_{a a}(p) \mathcal{S}(p)\right]^{n-2}
$$


With the sum

$$
\sum_{n=2}^{\infty}(n-1)(-x)^{n-2}=\frac{1}{(1+x)^{2}},
$$

and using (D.10) and (D.18) we obtain

$$
\mathcal{C}_{2}=2 I_{2}(p)^{2} H_{a b}(p)^{2}\left(1-4 I_{2}(p) B_{a a}^{\prime \prime}\right)^{2} .
$$

This reproduces the term (4.53).

We now turn to the diagrams of order $T$. First of all, note that the following two diagrams have no contributions proportional to $B_{a a}^{\prime \prime}$ :

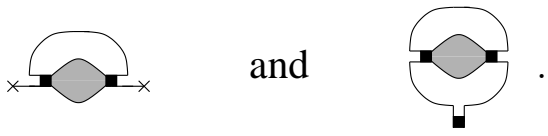

The reason is that they have no doubled line, as in

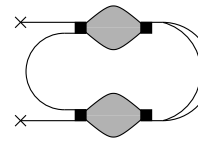

and

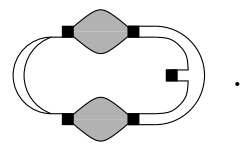

Note that both diagrams necessitate a replica-conserving double line (i.e. $\longrightarrow$ or $\longrightarrow$ ). Otherwise they vanish. The replica-conserving double line can be more generally replaced by all chains, which start and end with a double line, and which conserve the index running through. The chains with one index entering and one index exiting are

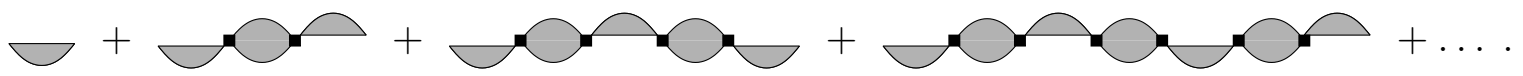

Each of these has the form (with $n+1$ half-chains)

$$
\mathcal{S}(p) \prod_{j=1}^{n}\left(\left(1-\delta_{i_{j} i_{j+1}}\right) \mathcal{S}(p) H_{i_{j} i_{j+1}}(p)\right) .
$$

The only replica-conserving term is obtained by using $\delta_{i_{j} i_{j+1}}$ in each factor. The projection onto the 1replica term is denoted $\mathcal{R}$ (for replica-conserving)

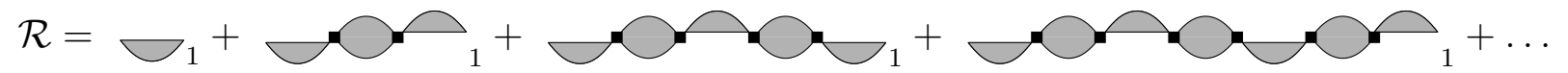

and is evaluated as

$$
\mathcal{R}=S(p) \sum_{n=0}^{\infty}\left[-\mathcal{S}(p) H_{a a}(p)\right]^{n}=\frac{\mathcal{S}(p)}{1+\mathcal{S}(p) H_{a a}(p)}=2 I_{2}(p)\left(1-4 I_{2}(p) B_{a a}^{\prime \prime}\right),
$$

where the last identity can be found in (D.19). This reestablishes the two factors of $\left(1-4 I_{2} B_{a a}^{\prime \prime}\right)$ in diagram (D.29). Note that $\mathcal{R}$ is $\curvearrowright$ introduced in section 4.2.

We can also give an equivalent interpretation of (D.21). It is $H_{a b}$ times summed half-chains, but since the indices are forced to be equal at the end, we can drop one of the $\delta$ 's in (D.31). Instead of (D.33) this is

$$
\begin{aligned}
\mathcal{T} & =\mathcal{S}(p) \sum_{n=0}^{\infty}\left[-\mathcal{S}(p) H_{a a}(p)\right]^{n}(1-n) \\
& =2 I_{2}(p)\left(1+2 I_{2}(p) B_{a a}^{\prime \prime}\right)\left(1-4 I_{2}(p) B_{a a}^{\prime \prime}\right),
\end{aligned}
$$

where the combinatorial factor $(1-n)$ is due to the fact, that one can drop one of the $\delta$ 's. We have also used the simplifications of equations (D.17) ff. $\mathcal{T}$ is nothing but $\triangle$, introduced in section 4.2. 


\section{E The 2-loop diagram with a tadpole and graphical interpretation of perturbation theory}

For simplicity of notations, this calculation is done for a 1-component field $u$. We also note $R(u):=B\left(u^{2}\right)$. We here calculate the 2-loop diagrams at finite temperature. This shows how the naive rules one uses at zero temperature can be misinterpreted.

All diagrams have two vertices $R$, two lines between these two $R$ 's, and a tadpole attached to one of the $R$ 's:
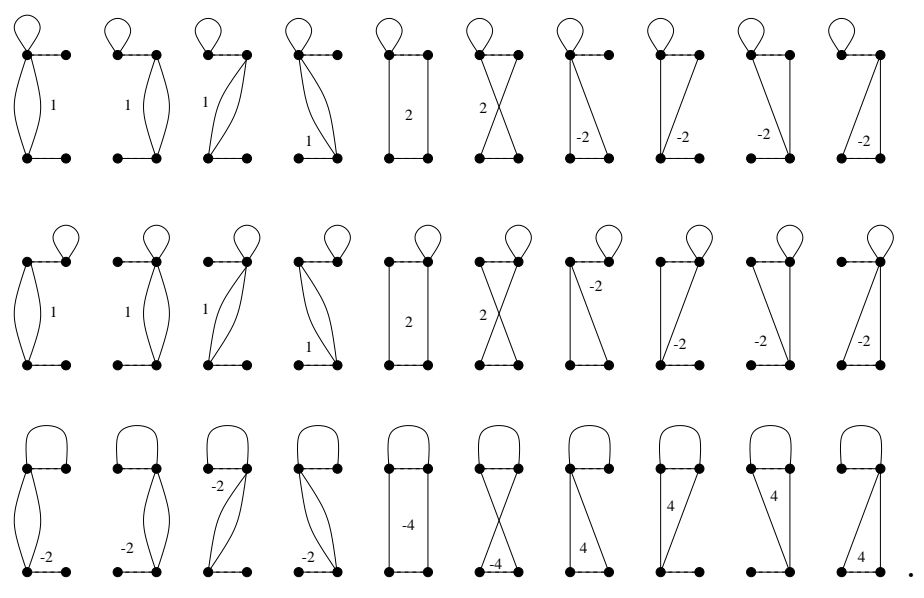

This is a graphical representation of $R_{a b}^{\prime \prime \prime \prime} R_{c d}^{\prime \prime}\left(\delta_{a a}+\delta_{b b}-2 \delta_{a b}\right)\left(\delta_{a c} \delta_{b d}+\delta_{a d} \delta_{b c}\right)^{2}$, together with combinatorial factors. Projecting onto 2-replica-terms only gives:

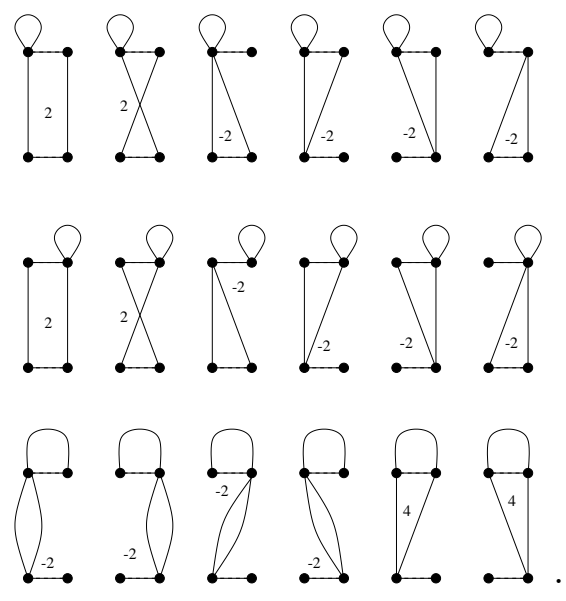


The interesting diagrams proportional to $R^{\prime \prime \prime \prime}(0)$ are:
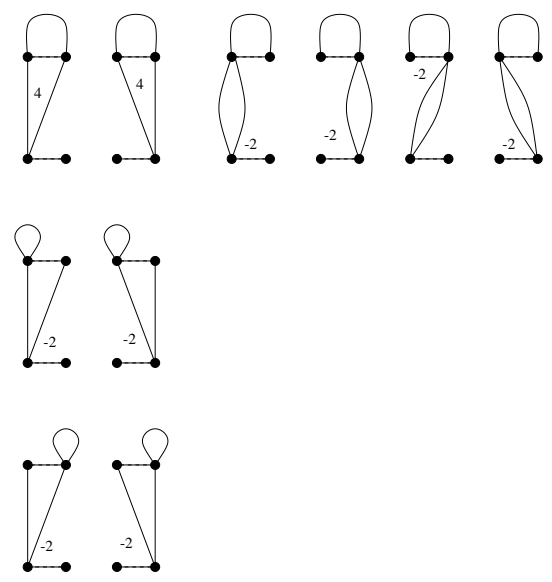

One sees that one has cancellation either in one column or in one line. One can interpret this as follows: Making first contractions between the two $R$ 's, identifying the replicas of the upper $R$, one can say that no tadpole can be added to the upper $R$. This is the cancellation in the first column. Equivalently, one can first draw the tadpole. If one does this connecting replicas $a$ and $b$ on the upper $R$, then one can say that it is no longer possible to add correlators connecting the two $R$ 's. This is the cancellation in the first line, as opposed to the first column.

\section{F Details of the algebraic method}

\section{F.1 Algebra of 4-replica matrices}

The 4-replica matrices needed in the $t r \ln$ calculation of Section 5 can be parameterized as in (5.5) and (5.10). They form an algebra, and to solve Eq. (5.11) one needs to write explicitly the components of an arbitrary product of such matrices.

Let us consider two matrices $M^{1}$ and $M^{2}$ parameterized respectively by $\left(x_{a}^{i}, y_{a b}^{i}, z_{a b}^{i}, t_{a b c}^{i}, u_{a b}^{i}, v_{a b c}^{i}, w_{a b c}^{i}\right.$, $\left.g_{a b c d}^{i}\right)$ for $i=1,2$. Then the product $M_{a b, c d}=\sum_{e f} M_{a b, e f}^{1} M_{e f, c d}^{2}$ is itself parameterized by $\left(x_{a}, y_{a b}, z_{a b}, t_{a b c}\right.$, $\left.u_{a b}, v_{a b c}, w_{a b c}, g_{a b c d}\right)$ and one finds:

$$
\begin{aligned}
x_{a}= & x_{a}^{1} x_{a}^{2}+2 y_{a a}^{1} x_{a}^{2}+2 x_{a}^{1} z_{a a}^{2}+2 \sum_{f} y_{a f}^{1} z_{a f}^{2} \\
y_{a d}= & x_{a}^{1} y_{a d}^{2}+2 x_{a}^{1} t_{a a d}^{2}+2 y_{a a}^{1} y_{a d}^{2}+2 \sum_{f} y_{a f}^{1} t_{a f d}^{2} \\
z_{a b}= & z_{a b}^{1} x_{a}^{2}+2 t_{a b a}^{1} x_{a}^{2}+2 z_{a b}^{1} z_{a a}^{2}+2 \sum_{f} t_{a b f}^{1} z_{a f}^{2} \\
t_{a b d}= & z_{a b}^{1} y_{a d}^{2}+2 t_{a b a}^{1} y_{a d}^{2}+2 z_{a b}^{1} t_{a a d}^{2}+2 \sum_{f} t_{a b f}^{1} t_{a f d}^{2} \\
u_{a c}= & x_{a}^{1} u_{a c}^{2}+u_{a c}^{1} x_{c}^{2}+v_{a c c}^{1} x_{c}^{2}+x_{a}^{1} w_{a a c}^{2}+2 y_{a c}^{1} z_{c a}^{2}+2 y_{a a}^{1} u_{a c}^{2}+2 u_{a c}^{1} z_{c c}^{2} \\
& +\sum_{f}\left[2 y_{a f}^{1} w_{a f c}^{2}+2 v_{a c f}^{1} z_{c f}^{2}+u_{a f}^{1} u_{f c}^{2}+v_{a f f}^{1} u_{f c}^{2}+u_{a f}^{1} w_{f f c}^{2}\right]+\sum_{e f} v_{a e f}^{1} w_{e f c}^{2} \\
v_{a c d}= & x_{a}^{1} v_{a c d}^{2}+x_{a}^{1} g_{a a c d}^{2}+2 y_{a c}^{1} t_{c a d}^{2}+2 y_{a d}^{1} t_{d a c}^{2}+u_{a c}^{1} y_{c d}^{2}+u_{a d}^{1} y_{d c}^{2}+2 y_{a a}^{1} v_{a c d}^{2}+v_{a c c}^{1} y_{c d}^{2}+v_{a d d}^{1} y_{d c}^{2} \\
& +2 u_{a c}^{1} t_{c c d}^{2}+2 u_{a d}^{1} t_{d d c}^{2}
\end{aligned}
$$




$$
\begin{aligned}
& +\sum_{f}\left[2 y_{a f}^{1} g_{a f c d}^{2}+2 v_{a c f}^{1} t_{c f d}^{2}+2 v_{a d f}^{1} t_{d f c}^{2}+u_{a f}^{1} v_{f c d}^{2}+u_{a f}^{1} g_{f f c d}^{2}+v_{a f f}^{1} v_{f c d}^{2}\right]+\sum_{e f} v_{a e f}^{1} g_{e f c d}^{2} \\
w_{a b c}= & w_{a b c}^{1} x_{c}^{2}+g_{a b c c}^{1} x_{c}^{2}+2 t_{a b c}^{1} z_{c a}^{2}+2 t_{b a c}^{1} z_{c b}^{2}+z_{a b}^{1} u_{a c}^{2}+z_{b a}^{1} u_{b c}^{2}+z_{a b}^{1} w_{a a c}^{2}+z_{b a}^{1} w_{b b c}^{2}+2 w_{a b c}^{1} z_{c c}^{2} \\
& +2 t_{a b a}^{1} u_{a c}^{2}+2 t_{b a b}^{1} u_{b c}^{2} \\
& +\sum_{f}\left[2 g_{a b c f}^{1} z_{c f}^{2} 2 t_{a b f}^{1} w_{a f c}^{2}+2 t_{b a f}^{1} w_{b f c}^{2}+w_{a b f}^{1} u_{f c}^{2}+g_{a b f f}^{1} u_{f c}^{2}+w_{a b f}^{1} w_{f f c}^{2}\right]+\sum_{e f} g_{a b e f}^{1} w_{e f c}^{2} \\
g_{a b c d}= & w_{a b c}^{1} y_{c d}^{2}+w_{a b d}^{1} y_{d c}^{2}+g_{a b c c}^{1} y_{c d}^{2}+g_{a b d d}^{1} y_{d c}^{2}+z_{a b}^{1} v_{a c d}^{2}+z_{b a}^{1} v_{b c d}^{2}+z_{a b}^{1} g_{a a c d}^{2}+z_{b a}^{1} g_{b b c d}^{2} \\
& +2 t_{a b c}^{1} t_{c a d}^{2}+2 t_{b a c}^{1} t_{c b d}^{2}+2 t_{a b d}^{1} t_{d a c}^{2}+2 t_{b a d}^{1} t_{d b c}^{2}+2 t_{a b a}^{1} v_{a c d}^{2}+2 t_{b a b}^{1} v_{b c d}^{2}+2 w_{a b c}^{1} t_{c c d}^{2}+2 w_{a b d}^{1} t_{d d c}^{2} \\
& +\sum_{f}\left[2 t_{a b f}^{1} g_{a f c d}^{2}+2 t_{b a f}^{1} g_{b f c d}^{2}+2 g_{a b c f}^{1} t_{c f d}^{2}+2 g_{a b d f}^{1} t_{d f c}^{2}+w_{a b f}^{1} v_{f c d}^{2}+g_{a b f f}^{1} v_{f c d}^{2}+w_{a b f}^{1} g_{f f c d}^{2}\right] \\
& +\sum_{e f} g_{a b e f}^{1} g_{e f c d}^{2}
\end{aligned}
$$

where we have made replica sums explicit.

Using these multiplication rules one can rewrite Eq. (5.11) in terms of a set of nonlinear equations for the components of $M^{\lambda}$ in terms of the components of $\overline{\mathcal{M}}$. Unfortunately no closed solution seemed possible (except in some very special cases). The next step is thus to expand each component in number of replica sums as in (5.13). This results in a hierarchy of equations for components with increasing number of replica sum. For instance the zero-sum components behave under multiplication as in (F.1), dropping all terms with replica sums, and so on. These equations can be solved iteratively, as discussed in the text. For this, one needs the zero- and one-sum components of the matrix $\overline{\mathcal{M}}$, the calculation of which we now detail.

\section{F.2 Calculation of the matrix $M$}

We start by computing the matrix $M$ in (5.1). As in the following subsections there are two stages. First make all Kronecker-deltas explicit, then expand each term in the number of replica sums. As discussed in the text, all intermediate free sums over two or more replicas can be dropped.

A straightforward calculation from (2.6), for a model with only a bare second cumulant, gives:

$$
\begin{aligned}
M_{a b, c d} & =\left.\left(2 T \partial_{\chi_{a b}} \partial_{\chi_{c d}} U(\chi)\right)\right|_{\chi=\chi_{v}} \\
& =-\frac{2}{T}\left(\delta_{a b c d} \sum_{e} B_{a e}^{\prime \prime}+\delta_{a b} \delta_{c d} B_{a c}^{\prime \prime}-\left(\delta_{a c d}+\delta_{b c d}\right) B_{a b}^{\prime \prime}-\left(\delta_{a b c}+\delta_{a b d}\right) B_{c d}^{\prime \prime}+\left(\delta_{a c} \delta_{b d}+\delta_{b c} \delta_{a d}\right) B_{a b}^{\prime \prime}\right)
\end{aligned}
$$

where $B_{a b}^{\prime \prime}=B^{\prime \prime}\left(\tilde{\chi}_{v}^{a b}\right)$, and $\tilde{\chi}_{v}^{a b}=\chi_{v}^{a a}+\chi_{v}^{b b}-2 \chi_{v}^{a b} ; \chi_{v}^{a b}$ is given by its saddle point value (2.16). Note that since one takes everything at the saddle point at the end, which is symmetric in $a, b$, all expressions resulting from two derivatives are symmetric: $B_{a b}^{\prime \prime}=B_{b a}^{\prime \prime}$ (even if one chooses the fluctuating fields a priori non-symmetric).

The matrix $\tilde{\chi}_{v}^{a b}$ still contains explicit Kronecker-deltas. As in the main text, one writes

$$
\tilde{\chi}_{v}^{a b}=\tilde{\chi}_{a b}+\delta_{a b} \tilde{\chi}_{a},
$$

where $\tilde{\chi}_{a b}$ and $\tilde{\chi}_{a}$ contain no Kronecker delta, and are computed below. Then one sees that

$$
B^{\prime \prime}\left(\tilde{\chi}_{v}^{a b}\right)=\delta_{a b}\left[B^{\prime \prime}\left(\tilde{\chi}_{a a}+\tilde{\chi}_{a}\right)-B^{\prime \prime}\left(\tilde{\chi}_{a a}\right)\right]+B^{\prime \prime}\left(\tilde{\chi}_{a b}\right) .
$$


Inserting this form into (F.2) above one finds that the contribution of the piece $\delta_{a b}\left[B^{\prime \prime}\left(\tilde{\chi}_{a a}+\tilde{\chi}_{a}\right)-B^{\prime \prime}\left(\tilde{\chi}_{a a}\right)\right]$ cancels exactly and thus one obtains that $M$ is given by (F.2) but with now $B_{a b}^{\prime \prime}=B^{\prime \prime}\left(\tilde{\chi}_{a b}\right)$, i.e. the part with no Kronecker delta.

We can now continue the calculation from (2.16) by expanding in the number of replica sums. First we define:

$$
\begin{aligned}
\left(-2 T \partial \tilde{U}^{0}\right)_{a b} & =\delta_{a b} \sum_{c} U_{a c}-U_{a b} \\
U_{a b} & =\frac{2}{T} \tilde{B}_{a b}^{\prime}+\frac{2}{T^{2}} \sum_{g} \tilde{S}_{a b g}^{\prime}
\end{aligned}
$$

where $\tilde{B}_{a b}^{\prime}=\tilde{B}^{\prime}\left(v_{a b}^{2}\right)$ and similarly for the three-replica term (which will drop later on). From there and (2.16) we obtain, dropping all higher order sums, the expansions:

$$
\begin{aligned}
\tilde{\chi}_{a}= & -2 T I_{1}-2 T I_{2} \sum_{e} U_{a e} \\
\tilde{\chi}_{a b}= & v_{a b}^{2}+2 T I_{1}+2 T I_{2}\left[U_{a b}-\frac{1}{2}\left(U_{a a}+U_{b b}\right)+\frac{1}{2} \sum_{e}\left(U_{a e}+U_{b e}\right)\right] \\
& +2 T I_{3}\left[\sum_{e} U_{a b}\left(U_{a e}+U_{b e}\right)-\sum_{e} U_{a e} U_{e b}-\sum_{e}\left(U_{a a} U_{a e}+U_{b b} U_{b e}-\frac{1}{2} U_{a e} U_{e a}-\frac{1}{2} U_{b e} U_{e b}\right)\right]
\end{aligned}
$$

Thus in each of the $B_{a b}^{\prime \prime}=B^{\prime \prime}\left(\tilde{\chi}_{a b}\right)$ matrices appearing in (F.2) the argument can be Taylor expanded, i.e. as

$$
B_{a b}^{\prime \prime}=\bar{B}_{a b}^{\prime \prime}+\bar{B}_{a b}^{\prime \prime \prime} \sum_{f} O_{a b f}
$$

where we have defined $\bar{B}_{a b}^{\prime \prime}=B^{\prime \prime}\left(\bar{\chi}_{a b}\right), \bar{B}_{a b}^{\prime \prime \prime}=B^{\prime \prime \prime}\left(\bar{\chi}_{a b}\right)$ and

$$
\begin{aligned}
\bar{\chi}_{a b}= & v_{a b}^{2}+2 T I_{1}+4 I_{2}\left(\tilde{B}_{a b}^{\prime}-\frac{1}{2}\left(\tilde{B}_{a a}^{\prime}+\tilde{B}_{b b}^{\prime}\right)\right) \\
O_{a b f}= & \frac{4}{T} I_{2}\left(\tilde{S}_{a b f}^{\prime}-\frac{1}{2}\left(\tilde{S}_{a a f}^{\prime}+\tilde{S}_{b b f}^{\prime}\right)\right)+2 I_{2}\left(\tilde{B}_{a f}^{\prime}+\tilde{B}_{b f}^{\prime}\right) \\
& +\frac{8}{T} I_{3}\left[\tilde{B}_{a b}^{\prime}\left(\tilde{B}_{a f}^{\prime}+\tilde{B}_{b f}^{\prime}\right)-\tilde{B}_{a f}^{\prime} \tilde{B}_{e f}^{\prime}-\left(\tilde{B}_{a a}^{\prime} \tilde{B}_{a f}^{\prime}+\tilde{B}_{b b}^{\prime} \tilde{B}_{b f}^{\prime}-\frac{1}{2} \tilde{B}_{a f}^{\prime} \tilde{B}_{f a}^{\prime}-\frac{1}{2} \tilde{B}_{b f}^{\prime} \tilde{B}_{f b}^{\prime}\right)\right]
\end{aligned}
$$

It will turn out below that at the end we will only need $O_{a a f}=4 I_{2} \tilde{B}_{a f}^{\prime}$. We will not perform this expansion and replacement now. First, we turn to the calculation of the matrix $\bar{N}$ and perform the product $M \bar{N}^{q}$, keeping $B_{a b}^{\prime \prime}$ unspecified.

\section{F.3 Calculation of the matrix $\bar{N}^{q}$}

We now compute the second matrix, $\bar{N}^{q}$, expanded up to one free replica sum. One has:

$$
\begin{aligned}
\bar{N}_{a b, c d}^{q} & =v_{a} v_{d} \bar{G}_{b c}^{q}+v_{b} v_{c} \bar{G}_{a d}^{q}+T \bar{\Pi}_{a b, c d}^{q} \\
\bar{G}^{q} & =C(q) \delta+\sum_{n \geq 1} C(q)^{n+1}\left(-2 T \partial \tilde{U}^{0}\right)^{n}
\end{aligned}
$$




$$
\begin{aligned}
\bar{\Pi}_{a b, c d}^{q}= & J_{1,1}^{q} \delta_{a d} \delta_{b c}+\sum_{n \geq 1} J_{1, n+1}^{q}\left(\delta_{a d}\left(-2 T \partial \tilde{U}^{0}\right)_{b c}+\delta_{b c}\left(-2 T \partial \tilde{U}^{0}\right)_{a d}\right) \\
& +\sum_{m \geq 1, n \geq 1} J_{m+1, n+1}^{q}\left(-2 T \partial \tilde{U}^{0}\right)_{a d}^{m}\left(-2 T \partial \tilde{U}^{0}\right)_{b c}^{n} \\
J_{i, j}^{q}= & \int_{k} \frac{1}{\left(k^{2}+m^{2}\right)^{i}} \frac{1}{\left((q-k)^{2}+m^{2}\right)^{j}} .
\end{aligned}
$$

In addition to (F.6) we also need:

$$
\left(-2 T \partial \tilde{U}^{0}\right)_{a b}^{2}=-\sum_{e} U_{a b}\left(U_{a e}+U_{b e}\right)+\sum_{c} U_{a c} U_{c b}=-\frac{4}{T^{2}} \sum_{e} \tilde{B}_{a b}^{\prime}\left(\tilde{B}_{a e}^{\prime}+\tilde{B}_{b e}^{\prime}\right)+\frac{4}{T^{2}} \sum_{e} \tilde{B}_{a e}^{\prime} \tilde{B}_{e b}^{\prime} .
$$

Since $\left(-2 T \partial \tilde{U}^{0}\right)_{a b}^{3}$ etc. contains only at least 2-replica sums, it can be dropped. We define:

$$
\begin{aligned}
\bar{N}_{a b, c d}^{q}= & N_{a d, b c}^{q}+N_{b c, a d}^{q} \\
N_{a d, b c}^{q}= & \frac{1}{2} T J_{1,1}^{q} \delta_{a d} \delta_{b c}+C(q) \delta_{a d} v_{b} v_{c}+v_{a} v_{d}\left(C(q)^{2}\left(-2 T \partial \tilde{U}^{0}\right)_{b c}+C(q)^{3}\left(-2 T \partial \tilde{U}^{0}\right)_{b c}^{2}\right. \\
& +T \delta_{a d}\left(J_{1,2}^{q}\left(-2 T \partial \tilde{U}^{0}\right)_{b c}+J_{1,3}^{q}\left(-2 T \partial \tilde{U}^{0}\right)_{b c}^{2}\right)+\frac{1}{2} T J_{2,2}^{q}\left(-2 T \partial \tilde{U}^{0}\right)_{a d}\left(-2 T \partial \tilde{U}^{0}\right)_{b c} \\
& \left.+T J_{2,3}^{q}\left(-2 T \partial \tilde{U}^{0}\right)_{a d}\left(-2 T \partial \tilde{U}^{0}\right)_{b c}^{2}\right) .
\end{aligned}
$$

We obtain:

$$
\begin{aligned}
N_{a d, b c}^{q}= & \delta_{a d} \delta_{b c} L_{a b}^{1}+\delta_{a d} P_{a, b c}^{1}+\delta_{b c} P_{b, a d}^{2}-U_{b c} v_{a} v_{d} C(q)^{2} \\
& +\frac{1}{2} T J_{2,2}^{q} U_{a d} U_{b c}+\left(v_{a} v_{d} C(q)^{3}-T J_{2,3}^{q} U_{a d}\right) \sum_{f}\left[U_{b f} U_{f c}-U_{b c}\left(U_{b f}+U_{c f}\right)\right] \\
L_{a b}^{1}= & \frac{1}{2} T J_{1,1}^{q}+T J_{1,2}^{q} \sum_{f} U_{b f} \\
P_{a, b c}^{1}= & C(q) v_{b} v_{c}-T J_{1,2}^{q} U_{b c}+T J_{1,3}^{q} \sum_{f}\left[U_{b f} U_{c f}-U_{b c}\left(U_{b f}+U_{c f}\right)\right]-\frac{1}{2} T J_{2,2}^{q} U_{b c} \sum_{f} U_{a f} \\
P_{b, a d}^{2}= & v_{a} v_{d} C(q)^{2} \sum_{f} U_{b f}-\frac{1}{2} T J_{2,2}^{q} U_{a d} \sum_{f} U_{b f} .
\end{aligned}
$$

This yields:

$$
\begin{aligned}
\bar{N}_{a b, c d}^{q}= & \delta_{a d} \delta_{b c} L_{a b}^{q}+\delta_{a d} Q_{a, b c}^{q}+\delta_{b c} Q_{b, a d}^{q}-\frac{2}{T}\left(\tilde{B}_{b c}^{\prime} v_{a} v_{d}+\tilde{B}_{a d}^{\prime} v_{b} v_{c}\right) C(q)^{2}+\frac{4}{T} J_{2,2}^{q} \tilde{B}_{a d}^{\prime} \tilde{B}_{b c}^{\prime} \\
& +\frac{4}{T^{2}}\left(v_{a} v_{d} C(q)^{3}-2 J_{2,3}^{q} \tilde{B}_{a d}^{\prime}\right) \sum_{f}\left[\tilde{B}_{b f}^{\prime} \tilde{B}_{f c}^{\prime}-\tilde{B}_{b c}^{\prime}\left(\tilde{B}_{b f}^{\prime}+\tilde{B}_{c f}^{\prime}\right)\right] \\
& +\frac{4}{T^{2}}\left(v_{b} v_{c} C(q)^{3}-2 J_{2,3}^{q} \tilde{B}_{b c}^{\prime}\right) \sum_{f}\left[\tilde{B}_{a f}^{\prime} \tilde{B}_{f d}^{\prime}-\tilde{B}_{a d}^{\prime}\left(\tilde{B}_{a f}^{\prime}+\tilde{B}_{d f}^{\prime}\right)\right] \\
L_{a b}^{q}= & T J_{1,1}^{q}+2 J_{1,2}^{q} \sum_{f}\left(\tilde{B}_{a f}^{\prime}+\tilde{B}_{b f}^{\prime}\right) \\
Q_{a, b c}^{q}= & v_{b} v_{c}\left(C(q)+\frac{2}{T} C(q)^{2} \sum_{f} \tilde{B}_{a f}^{\prime}\right)-\frac{2}{T} \tilde{B}_{b c}^{\prime}\left(T J_{1,2}^{q}+2 J_{2,2}^{q} \sum_{f} \tilde{B}_{a f}^{\prime}\right)
\end{aligned}
$$




$$
+\frac{4}{T} J_{1,3}^{q} \sum_{f}\left[\tilde{B}_{b f}^{\prime} \tilde{B}_{c f}^{\prime}-\tilde{B}_{b c}^{\prime}\left(\tilde{B}_{b f}^{\prime}+\tilde{B}_{c f}^{\prime}\right)\right]
$$

\section{F.4 Final calculation of the matrix $\mathcal{M}$}

We now perform the matrix multiplication

$$
\mathcal{M}_{a b, c d}^{q}=\sum_{e f} M_{a b, e f} \bar{N}_{e f, c d}^{q}=-\frac{2}{T}\left[B_{a b}^{\prime \prime} \mathcal{N}_{a b, c d}^{q}-\delta_{a b} \sum_{g} B_{a g}^{\prime \prime} \mathcal{N}_{a g, c d}^{q}\right],
$$

where we have defined:

$$
\begin{aligned}
\mathcal{N}_{a b, c d}^{q}= & \left(\bar{N}_{a b, c d}^{q}+\bar{N}_{b a, c d}^{q}-\bar{N}_{a a, c d}^{q}-\bar{N}_{b b, c d}^{q}\right)=L_{a b}^{q}\left(\delta_{a d} \delta_{b c}+\delta_{b d} \delta_{a c}\right)-L_{a a}^{q} \delta_{a c d}-L_{b b}^{q} \delta_{b c d} \\
& +\delta_{a d} Q_{a, b c}^{q}+\delta_{b d} Q_{b, a c}^{q}-\delta_{a d} Q_{a, a c}^{q}-\delta_{b d} Q_{b, b c}^{q}+\delta_{b c} Q_{b, a d}^{q}+\delta_{a c} Q_{a, b d}^{q}-\delta_{a c} Q_{a, a d}^{q}-\delta_{b c} Q_{b, b d}^{q} \\
& -\left(U_{b c} v_{a} v_{d}+U_{a d} v_{b} v_{c}+U_{a c} v_{b} v_{d}+U_{b d} v_{a} v_{c}-U_{a c} v_{a} v_{d}-U_{a d} v_{a} v_{c}-U_{b c} v_{b} v_{d}-U_{b d} v_{b} v_{c}\right) I_{2}^{q} \\
& +T J_{2,2}^{q}\left(U_{a d} U_{b c}+U_{b d} U_{a c}-U_{a d} U_{a c}-U_{b d} U_{b c}\right) \\
& +\left(v_{a} v_{d} C(q)^{3}-T J_{2,3}^{q} U_{a d}\right) \sum_{f}\left[U_{b f} U_{f c}-U_{b c}\left(U_{b f}+U_{c f}\right)\right] \\
& +\left(v_{b} v_{c} C(q)^{3}-T J_{2,3}^{q} U_{b c}\right) \sum_{f}\left[U_{a f} U_{f d}-U_{a d}\left(U_{a f}+U_{d f}\right)\right] \\
& +\left(v_{b} v_{d} C(q)^{3}-T J_{2,3}^{q} U_{b d}\right) \sum_{f}\left[U_{a f} U_{f c}-U_{a c}\left(U_{a f}+U_{c f}\right)\right] \\
& +\left(v_{a} v_{c} C(q)^{3}-T J_{2,3}^{q} U_{a c}\right) \sum_{f}\left[U_{b f} U_{f d}-U_{b d}\left(U_{b f}+U_{d f}\right)\right] \\
& -\left(v_{a} v_{d} C(q)^{3}-T J_{2,3}^{q} U_{a d}\right) \sum_{f}\left[U_{a f} U_{f c}-U_{a c}\left(U_{a f}+U_{c f}\right)\right] \\
& -\left(v_{a} v_{c} C(q)^{3}-T J_{2,3}^{q} U_{a c}\right) \sum_{f}\left[U_{a f} U_{f d}-U_{a d}\left(U_{a f}+U_{d f}\right)\right] \\
& -\left(v_{b} v_{d} C(q)^{3}-T J_{2,3}^{q} U_{b d}\right) \sum_{f}\left[U_{b f} U_{f c}-U_{b c}\left(U_{b f}+U_{c f}\right)\right] \\
& -\left(v_{b} v_{c} C(q)^{3}-T J_{2,3}^{q} U_{b c}\right) \sum_{f}\left[U_{b f} U_{f d}-U_{b d}\left(U_{b f}+U_{d f}\right)\right]
\end{aligned}
$$

Performing the matrix product yields the parameterization of the matrix $\overline{\mathcal{M}}$, where we have not yet fully expanded in sums, as (the $q$-dependence is implicit):

$$
\begin{aligned}
\alpha_{a b} & =-\frac{2}{T} \frac{1}{1-\frac{4}{T} B_{a b}^{\prime \prime} L_{a b}} \\
x_{a} & =\alpha_{a a} \sum_{f} B_{a f}^{\prime \prime} L_{a a} \\
y_{a d} & =-\alpha_{a a} B_{a d}^{\prime \prime} L_{a d}+\alpha_{a a} \sum_{f} B_{a f}^{\prime \prime}\left(Q_{a, a d}-Q_{a, f d}\right) \\
z_{a b} & =-\alpha_{a b} B_{a b}^{\prime \prime} L_{a a}
\end{aligned}
$$




$$
\begin{aligned}
& t_{a b d}=\alpha_{a b} B_{a b}^{\prime \prime}\left(Q_{a, b d}-Q_{a, a d}\right) \\
& u_{a c}=\alpha_{a a} B_{a c}^{\prime \prime} L_{c c} \\
& v_{a c d}=\alpha_{a a}\left[B_{a d}^{\prime \prime}\left(Q_{d, d c}-Q_{d, a c}\right)+B_{a c}^{\prime \prime}\left(Q_{c, c d}-Q_{c, a d}\right)\right. \\
& -\sum_{f} B_{a f}^{\prime \prime} T J_{2,2}^{q}\left(U_{a d} U_{f c}+U_{f d} U_{a c}-U_{a d} U_{a c}-U_{f d} U_{f c}\right) \\
& +\sum_{f} B_{a f}^{\prime \prime}\left(U_{f c} v_{a} v_{d}+U_{a d} v_{f} v_{c}+U_{a c} v_{f} v_{d}+U_{f d} v_{a} v_{c}\right. \\
& \left.\left.-U_{a c} v_{a} v_{d}-U_{a d} v_{a} v_{c}-U_{f c} v_{f} v_{d}-U_{f d} v_{f} v_{c}\right) I_{2}^{q}\right] \\
& w_{a b c}=0 \\
& g_{a b c d}=\alpha_{a b} B_{a b}^{\prime \prime}\left\{-\left(U_{b c} v_{a} v_{d}+U_{a d} v_{b} v_{c}+U_{a c} v_{b} v_{d}+U_{b d} v_{a} v_{c}\right.\right. \\
& \left.-U_{a c} v_{a} v_{d}-U_{a d} v_{a} v_{c}-U_{b c} v_{b} v_{d}-U_{b d} v_{b} v_{c}\right) I_{2}^{q} \\
& +T J_{2,2}^{q}\left(U_{a d} U_{b c}+U_{b d} U_{a c}-U_{a d} U_{a c}-U_{b d} U_{b c}\right) \\
& +\left(v_{a} v_{d} I_{3}^{q}-T J_{2,3}^{q} U_{a d}\right) \sum_{f}\left[U_{b f} U_{f c}-U_{b c}\left(U_{b f}+U_{c f}\right)\right] \\
& +\left(v_{b} v_{c} I_{3}^{q}-T J_{2,3}^{q} U_{b c}\right) \sum_{f}\left[U_{a f} U_{f d}-U_{a d}\left(U_{a f}+U_{d f}\right)\right] \\
& +\left(v_{b} v_{d} I_{3}^{q}-T J_{2,3}^{q} U_{b d}\right) \sum_{f}\left[U_{a f} U_{f c}-U_{a c}\left(U_{a f}+U_{c f}\right)\right] \\
& +\left(v_{a} v_{c} I_{3}^{q}-T J_{2,3}^{q} U_{a c}\right) \sum_{f}\left[U_{b f} U_{f d}-U_{b d}\left(U_{b f}+U_{d f}\right)\right] \\
& -\left(v_{a} v_{d} I_{3}^{q}-T J_{2,3}^{q} U_{a d}\right) \sum_{f}\left[U_{a f} U_{f c}-U_{a c}\left(U_{a f}+U_{c f}\right)\right] \\
& -\left(v_{a} v_{c} I_{3}^{q}-T J_{2,3}^{q} U_{a c}\right) \sum_{f}\left[U_{a f} U_{f d}-U_{a d}\left(U_{a f}+U_{d f}\right)\right] \\
& -\left(v_{b} v_{d} I_{3}^{q}-T J_{2,3}^{q} U_{b d}\right) \sum_{f}\left[U_{b f} U_{f c}-U_{b c}\left(U_{b f}+U_{c f}\right)\right] \\
& \left.-\left(v_{b} v_{c} I_{3}^{q}-T J_{2,3}^{q} U_{b c}\right) \sum_{f}\left[U_{b f} U_{f d}-U_{b d}\left(U_{b f}+U_{d f}\right)\right]\right\} .
\end{aligned}
$$

We now finish the expansion in sums, using (F.9), (F.11) and defining the notations:

$$
\begin{aligned}
A_{a b}^{q} & =\frac{2}{1-4 \bar{B}_{a b}^{\prime \prime} J_{1,1}^{q}} \\
A_{a a}^{q} & =\frac{2}{1-4 B^{\prime \prime}\left(2 T I_{1}\right) J_{1,1}^{q}} \\
\alpha_{a b} & =-\frac{1}{T}\left[A_{a b}^{q}+2\left(A_{a b}^{q}\right)^{2}\left(\frac{2}{T} J_{1,2}^{q} \bar{B}_{a b}^{\prime \prime} \sum_{f}\left(\tilde{B}_{a f}^{\prime}+\tilde{B}_{b f}^{\prime}\right)+J_{1,1}^{q} \bar{B}_{a b}^{\prime \prime \prime} \sum_{f} O_{a b f}\right)\right] \\
L_{a b} & =T J_{1,1}^{q}+2 J_{1,2}^{q} \sum_{f}\left(\tilde{B}_{a f}^{\prime}+\tilde{B}_{b f}^{\prime}\right)
\end{aligned}
$$



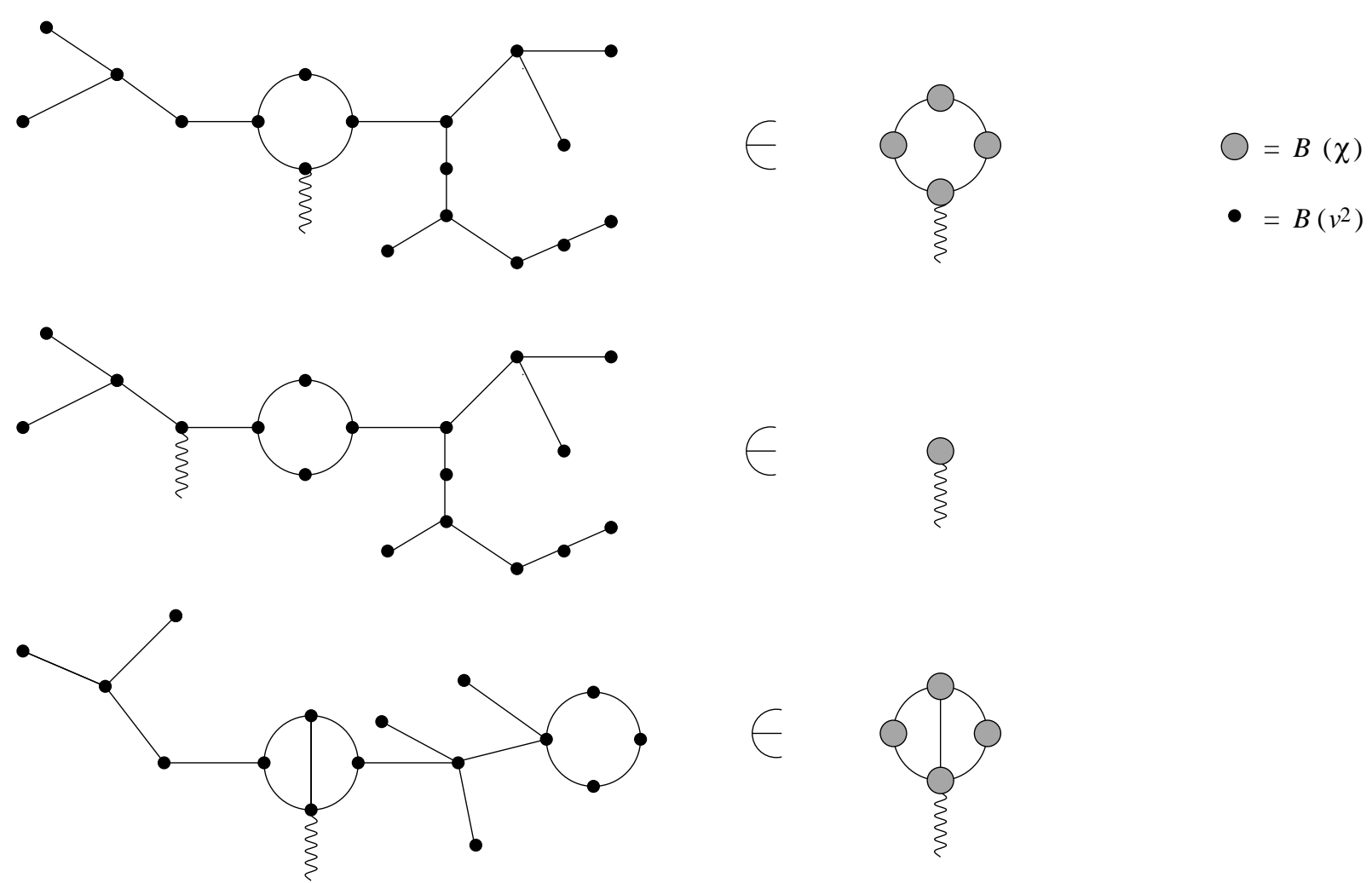

Figure 6: Some typical $1 / N$-diagrams and the classes they belong to. The wiggly line indicates the derivative, the black circle is a $\tilde{B}$ (or derivative), the grey circle a $B$ (or derivative). We have restrained from drawing a contribution due to the explicit $v$-dependence. These terms do not play a role in the argument, and are only tedious supplements one has to keep track of.

$\mathrm{h}$ The result is given in the text in section 5 , as well as:

$$
\begin{aligned}
P_{1} y_{a d}= & 2 J_{1,1}^{q}\left(A_{a a}^{q}\right)^{2}\left(\frac{4}{T} J_{1,2}^{q} \bar{B}_{a a}^{\prime \prime} \sum_{f} \tilde{B}_{a f}^{\prime}+J_{1,1}^{q} \bar{B}_{a a}^{\prime \prime \prime} \sum_{f} O_{a a f}\right) \bar{B}_{a d}^{\prime \prime}+\frac{2}{T} A_{a a} J_{1,2}^{q} \bar{B}_{a d}^{\prime \prime} \sum_{f}\left(\tilde{B}_{a f}^{\prime}+\tilde{B}_{d f}^{\prime}\right) \\
& +J_{1,1}^{q} A_{a a}^{q} \bar{B}_{a d}^{\prime \prime \prime} \sum_{f} O_{a d f}-\frac{2}{T} \frac{C(q)}{\left(1-4 B^{\prime \prime}\left(2 T I_{1}\right) J_{1,1}^{q}\right)} \sum_{f} \bar{B}_{a f}^{\prime \prime}\left(v_{a}-v_{f}\right) v_{d} \\
P_{1} z_{a b}= & 2 J_{1,1}^{q}\left(A_{a b}^{q}\right)^{2}\left(\frac{2}{T} J_{1,2}^{q} \bar{B}_{a b}^{\prime \prime} \sum_{f}\left(\tilde{B}_{a f}^{\prime}+\tilde{B}_{b f}^{\prime}\right)+J_{1,1}^{q} \bar{B}_{a b}^{\prime \prime \prime} \sum_{f} O_{a b f}\right) \bar{B}_{a b}^{\prime \prime} \\
& +\frac{4}{T} A_{a b} J_{1,2}^{q} b_{a b}^{\prime \prime} \sum_{f} \tilde{B}_{a f}^{\prime}+J_{1,1}^{q} A_{a b}^{q} \bar{B}_{a b}^{\prime \prime \prime} \sum_{f} O_{a b f}
\end{aligned}
$$

\section{G More remarks on the graphical method}

\section{G.1 Diagrammatics}

The diagrammatic $1 / N$ expansion can be constructed by using the Theorem:

$$
\tilde{B}^{\prime}\left(v^{2}\right)=\left[\frac{\partial}{\partial \chi}+\frac{\partial}{\partial v^{2}}\right]\left(B(\chi)+\frac{1}{N} B^{(1)}\left(\chi(v), v^{2}\right)+\frac{1}{N^{2}} B^{(2)}\left(\chi(v), v^{2}\right)+\ldots\right)
$$




\section{$0000000000 \otimes \theta$}

Figure 7: Diagrams at order $1 / N$ (first), $1 / N^{2}$ (next two) and $1 / N^{3}$ (rest).

$$
\begin{aligned}
B^{(1)}(\chi) & =\sum \text { all 1PI diagrams with } 1 \text { loop } \\
B^{(2)}(\chi) & =\sum \text { all 1PI diagrams with } 2 \text { loops } \\
\ldots & =\ldots \\
\chi(v) & =v^{2}+2 T I_{1}+4 I_{2}\left[\partial_{\chi} B\left(\chi\left(v^{2}\right), v^{2}\right)-\partial_{\chi} B(\chi(0), 0)\right] .
\end{aligned}
$$

Some explication and precisions are in order: 1-particle-irreducible diagrams (1PI) are w.r.t. lines being correlators $\langle v v\rangle$, and vertices being $B^{(n)}(\chi)$. The r.h.s. of Eq. (G.1) are diagrams made out of bare vertices. We have separated the $\chi$-dependence from the explicit $v$-dependence: The latter are $v$ 's which are connected with a line. These are the terms in our $1 / N$-calculation, which explicitly contain $v$. Note that $v$ 's always pair. Side-chains only come from the fact that finally one inserts $\chi$. Note that $\chi$ as defined here is an object which contains terms at all orders in $1 / N$. The diagrams are $1 \mathrm{PI}$, a fact which is important for the order $1 / N^{2}$. It means that $B^{(2)}$ does not contain the diagram made out of 2 closed loops, connected by a single line.

Proof:

Draw a collection of diagrams contributing to $\tilde{B}$ (see figure 6). This drawing contains vertices made out of derivatives of $B\left(v^{2}\right)$ (not $B(\chi)$ - we have drawn the completely expanded diagram). Now derive that object with respect to $v^{2}$, giving a couple of terms. Any of these terms singles out one $B$, namely the one derived.

This $B$ may be part of a tree, by which we mean that either it is a point or by cutting off one of the attached legs, the diagram will fall apart. Then it is contained in the first term on the r.h.s. of Eq. (G.1), since any attachment which can be made to it in the form of a tree, is taking care of by choosing the above given $\chi$. Note that for this to be true, $\chi$ has to be exactly the object given above, i.e. on the r.h.s. of Eq. (G.5) there has to be the full $\tilde{B}$ to all orders in $1 / N$. In the diagrammatic language this is clear: Having a higher-order diagram and taking the derivative at one of the tree-like vertices, this diagram may still contain an arbitrary loop somewhere attached to the tree.

The $B$ which has been derived may as well be part of a closed loop. By this we mean that when we cut off all parts of the diagram which can be disconnect from our chosen one by one cut, there remains more than the vertex itself. This object is of higher connectivity; it can either be a loop (at order $1 / N$ ); it can be a diagram in the form of an 8 or a circle to which one has added an additional line between two arbitrary chosen points on it (at order $1 / N^{2}$ ). Higher order diagrams are given in figure 7 .

\section{G.2 An alternative derivation of the $\beta$-function $(T=0)$}

We now give a general derivation of the $\beta$-function to all orders in $1 / N$, following our results of the last section. This derivation is restricted to $T=0$, since it is rather complicated at finite $T$. To make the derivation more transparent, and to avoid having to derive with respect to $v^{2}$ on the r.h.s. of Eq. (G.1), we introduce the auxiliary function $\tilde{B}\left(v^{2}, u^{2}\right)$. By $u^{2}$ we shall denote a pair of background-fields that are non-trivially connected by a line of propagators, whereas $v^{2}$ denotes the background-field which is inserted into $B$, but which is not connected to any propagator. Note that this decomposition is unique; that the paring of $u$ 's is natural, and that deriving with respect to $v^{2}$, but not $u^{2}$ can combinatorically be interpreted as choosing any vertex $B$ and deriving it once. This object is thus better fitted for calculations 
than $\tilde{B}\left(v^{2}\right)=\left.\tilde{B}\left(v^{2}, u^{2}\right)\right|_{u^{2}=v^{2}}$. However the latter object is of course the only one with a physical meaning.

We now start from a modified version of Eq. (G.1), namely

$$
\begin{aligned}
\partial_{v^{2}} \tilde{B}\left(v^{2}, u^{2}\right) & =\frac{\partial}{\partial \chi}\left(B(\chi)+\delta B\left(\chi, u^{2}\right)\right) \\
\delta B\left(\chi, u^{2}\right) & =\delta B\left[B^{\prime}(\chi(v)), B^{\prime \prime}(\chi(v)), \ldots, u^{2}\right] \\
& =\frac{1}{N} B^{(1)}\left[B^{\prime}(\chi(v)), B^{\prime \prime}(\chi(v)), \ldots\right]+\frac{1}{N^{2}} B^{(2)}\left[B^{\prime}(\chi(v)), B^{\prime \prime}(\chi(v)), \ldots, u^{2}\right]+\ldots \\
\chi=\chi(v) & =\chi\left(v, u^{2}\right)=v^{2}+4 I_{2}\left(\partial_{v^{2}} \tilde{B}\left(v^{2}, u^{2}\right)-\partial_{v^{2}} \tilde{B}(0,0)\right) \\
& \equiv v^{2}+\frac{1}{\epsilon}\left(\partial_{v^{2}} \tilde{b}\left(v^{2}, u^{2}\right)-\partial_{v^{2}} b(0,0)\right),
\end{aligned}
$$

where we are a little bit sloppy with the notations, suppressing the argument $u$ of $\chi(v)$. We define the dimensionless quantities

$$
\begin{aligned}
b\left(v^{2}, u^{2}\right) & =4 \epsilon I_{2} \tilde{B}\left(v^{2}, u^{2}\right)=4 A_{d} m^{-\epsilon} \tilde{B}\left(v^{2}, u^{2}\right) \\
b_{0}(\chi) & =4 A_{d} m^{-\epsilon} B(\chi) \\
\delta b\left(\chi, u^{2}\right) & =4 A_{d} m^{-\epsilon} \delta B\left(\chi, u^{2}\right) .
\end{aligned}
$$

As in the main text, we use the notation $i_{n}(p)=\frac{I_{n}(p)}{A_{d}}=\frac{I_{n}(p)}{\epsilon I_{2}}$. The $\beta$-function is

$$
\begin{aligned}
-m \frac{\partial}{\partial m} \partial_{v^{2}} b\left(v^{2}, u^{2}\right)= & \epsilon \partial_{v^{2}} b\left(v^{2}, u^{2}\right)-\left(4 A_{d} m^{-\epsilon}\right) \frac{m \partial}{\partial m} \frac{\partial}{\partial \chi}\left(B(\chi)+\delta B\left(\chi, u^{2}\right)\right) \\
= & \epsilon \partial_{v^{2}} b\left(v^{2}, u^{2}\right)+\frac{\partial^{2}}{\partial \chi^{2}}\left(b(\chi)+\delta b\left(\chi, u^{2}\right)\right)\left(-m \frac{\partial \chi}{\partial m}\right) \\
& +\left.\epsilon \frac{\partial}{\partial \lambda}\right|_{\lambda=1}\left[\frac{1}{\lambda} \frac{\partial}{\partial \chi} \delta b\left(\lambda b_{0}^{\prime}, \lambda b_{0}^{\prime \prime}, \ldots, u^{2}\right)\right] .
\end{aligned}
$$

Note that in the last equation, we have been a little bit sloppy with the notation. What this means is that having rescaled $B$ to $b_{0}$, the $m$-dependence of the integrals is canceled. Thus we can evaluate all integrals at $m^{2}=1$. The derivative w.r.t. $\lambda$ is easily understood as follows: Having a diagram with $n+1$ vertices, the integrals scale like $m^{-n \epsilon}$. First, this accounts for the factor of $\epsilon$. Second, in order to get the right combinatorial factor of $n$ instead of $n+1$, one has to subtract one contribution, which is done by the factor of $1 / \lambda$ in front of $\delta b$.

We need two more equations. First, starting from Eq. (G.8) and deriving w.r.t. $m$, we obtain (exact!)

$$
-m \frac{\partial \chi}{\partial m}=\frac{1}{\epsilon}\left(-m \frac{\partial}{\partial m}\right)\left[\partial_{v^{2}} b\left(v^{2}, u^{2}\right)-\partial_{v^{2}} b(0,0)\right] \neq \frac{1}{\epsilon}\left(-m \frac{\partial}{\partial m}\right) \partial_{v^{2}} b\left(v^{2}, u^{2}\right)-\partial_{v^{2}} b(0,0),
$$

where an equality would suppose that due to dimensional reduction $\partial_{m} \tilde{B}^{\prime}(0)=0$.

Deriving Eq. (G.8) w.r.t. $v^{2}$, we obtain (also exact)

$$
\frac{\partial \chi}{\partial v^{2}}=1+\frac{1}{\epsilon} \partial_{v^{2}}^{2} b\left(v^{2}, u^{2}\right) \text {. }
$$

Deriving Eq. (G.6) by $v^{2}$ gives with the help of Eq. (G.14)

$$
\begin{aligned}
\partial_{v^{2}}^{2} b\left(v^{2}, u^{2}\right) & =\frac{\partial^{2}}{\partial \chi^{2}}\left[b_{0}(\chi)+\delta b\left(\chi, u^{2}\right)\right] \frac{\partial \chi}{\partial v^{2}} \\
& =\frac{\partial^{2}}{\partial \chi^{2}}\left[b_{0}(\chi)+\delta b\left(\chi, u^{2}\right)\right]\left(1+\frac{1}{\epsilon} \partial_{v^{2}}^{2} b\left(v^{2}, u^{2}\right)\right) .
\end{aligned}
$$


Therefrom we infer that (to all orders)

$$
\frac{\partial^{2}}{\partial \chi^{2}}\left[b_{0}(\chi)+\delta b\left(\chi, u^{2}\right)\right]=\frac{\partial_{v^{2}}^{2} b\left(v^{2}, u^{2}\right)}{1+\frac{1}{\epsilon} \partial_{v^{2}}^{2} b\left(v^{2}, u^{2}\right)} .
$$

This equation can also be written as

$$
\frac{1}{\frac{\partial^{2}}{\partial \chi^{2}}\left[b_{0}(\chi)+\delta b\left(\chi, u^{2}\right)\right]}=\frac{1}{\partial_{v^{2}}^{2} b\left(v^{2}, u^{2}\right)}+\frac{1}{\epsilon} .
$$

This procedure can be repeated to obtain

$$
\begin{aligned}
\frac{\partial^{3}}{\partial \chi^{3}}\left[b_{0}(\chi)+\delta b\left(\chi, u^{2}\right)\right] & =\frac{\partial_{v^{2}}^{3} b\left(v^{2}, u^{2}\right)}{\left(1+\frac{1}{\epsilon} \partial_{v^{2}}^{2} b\left(v^{2}, u^{2}\right)\right)^{3}} \\
\frac{\partial^{4}}{\partial \chi^{4}}\left[b_{0}(\chi)+\delta b\left(\chi, u^{2}\right)\right] & =\frac{\partial_{v^{2}}^{4} b\left(v^{2}, u^{2}\right)+\frac{1}{\epsilon}\left(\partial_{v^{2}}^{4} b\left(v^{2}, u^{2}\right) \partial_{v^{2}}^{2} b\left(v^{2}, u^{2}\right)-3\left[\partial_{v^{2}}^{3} b\left(v^{2}, u^{2}\right)\right]^{2}\right)}{\left(1+\frac{1}{\epsilon} \partial_{v^{2}}^{2} b\left(v^{2}, u^{2}\right)\right)^{5}} .
\end{aligned}
$$

Eliminating $\frac{\partial \chi}{\partial m}$ and $\frac{\partial^{2}}{\partial \chi^{2}}\left[b_{0}(\chi)+\delta b\left(\chi, u^{2}\right)\right]$ from Eq. (G.12), we obtain

$$
\begin{aligned}
-m \frac{\partial}{\partial m} \partial_{v^{2}} b\left(v^{2}, u^{2}\right)= & \epsilon \partial_{v^{2}} b\left(v^{2}, u^{2}\right)+\frac{\partial_{v^{2}}^{2} b\left(v^{2}, u^{2}\right)}{1+\frac{1}{\epsilon} \partial_{v^{2}}^{2} b\left(v^{2}, u^{2}\right)} \frac{1}{\epsilon}\left(-m \frac{\partial}{\partial m}\right)\left(\partial_{v^{2}} b\left(v^{2}, u^{2}\right)-\partial_{v^{2}} b(0,0)\right) \\
& +\left.\epsilon \frac{\partial}{\partial \lambda}\right|_{\lambda=1}\left[\frac{1}{\lambda} \frac{\partial}{\partial \chi} \delta b\left(\lambda b_{0}^{\prime}, \lambda b_{0}^{\prime \prime}, \ldots\right)\right] .
\end{aligned}
$$

We now take the limit of $v^{2}, u^{2} \rightarrow 0$. We suppose that $\left(-m \frac{\partial}{\partial m}\right)\left(\partial_{v^{2}} b\left(v^{2}, u^{2}\right)-\partial_{v^{2}} b(0,0)\right) \rightarrow 0$ in that limit. Further $\partial_{v^{2}}^{2} b\left(v^{2}, u^{2}\right)$ can either remain finite or diverge. However $\frac{\partial_{v}^{2} b\left(v^{2}, u^{2}\right)}{1+\frac{1}{\epsilon} \partial_{v^{2}}^{2} b\left(v^{2}, u^{2}\right)}$ remains finite whatever $\partial_{v^{2}}^{2} b\left(v^{2}, u^{2}\right)$ will do. Supposing that this argument is indeed correct (are there additional IR-divergences?) the conclusion is that

$$
-m \frac{\partial}{\partial m} \partial_{v^{2}} b(0,0)=\epsilon \partial_{v^{2}} b(0,0)+\left.\lim _{u, v \rightarrow 0} \epsilon \frac{\partial}{\partial \lambda}\right|_{\lambda=1}\left[\frac{1}{\lambda} \frac{\partial}{\partial \chi} \delta b\left(\lambda b_{0}^{\prime}, \lambda b_{0}^{\prime \prime}, \ldots\right)\right] .
$$

The $\beta$-function thus is equivalent to

$$
\begin{aligned}
-m \frac{\partial}{\partial m} \partial_{v^{2}} b\left(v^{2}, u^{2}\right)= & \epsilon \partial_{v^{2}} b\left(v^{2}, u^{2}\right)+\left[\partial_{v^{2}} b\left(v^{2}, u^{2}\right)-\partial_{v^{2}} b(0,0)\right] \partial_{v^{2}}^{2} b\left(v^{2}, u^{2}\right) \\
& +\left.\left(\epsilon+\partial_{v^{2}}^{2} b\left(v^{2}, u^{2}\right)\right) \frac{\partial}{\partial \lambda}\right|_{\lambda=1}\left[\frac{1}{\lambda} \frac{\partial}{\partial \chi} \delta b\left(\lambda b_{0}^{\prime}, \lambda b_{0}^{\prime \prime}, \ldots\right)\right] \\
& -\left.\partial_{v^{2}}^{2} b\left(v^{2}, u^{2}\right) \lim _{v, u \rightarrow 0} \frac{\partial}{\partial \lambda}\right|_{\lambda=1}\left[\frac{1}{\lambda} \frac{\partial}{\partial \chi} \delta b\left(\lambda b_{0}, \lambda b_{0}^{\prime}, \ldots\right)\right] .
\end{aligned}
$$

Using Eq. (G.14), this can also be written as

$$
\begin{aligned}
-m \frac{\partial}{\partial m} \partial_{v^{2}} b\left(v^{2}, u^{2}\right)= & \epsilon \partial_{v^{2}} b\left(v^{2}, u^{2}\right)+\left[\partial_{v^{2}} b\left(v^{2}, u^{2}\right)-\partial_{v^{2}} b(0,0)\right] \partial_{v^{2}}^{2} b\left(v^{2}, u^{2}\right) \\
& +\left.\epsilon \frac{\partial}{\partial \lambda}\right|_{\lambda=1} \frac{\partial}{\partial v^{2}}\left[\frac{1}{\lambda} \delta b\left(\lambda b_{0}^{\prime}, \lambda b_{0}^{\prime \prime}, \ldots\right)\right] \\
& -\left.\partial_{v^{2}}^{2} b\left(v^{2}, u^{2}\right) \lim _{v, u \rightarrow 0} \frac{\partial}{\partial \lambda}\right|_{\lambda=1}\left[\frac{1}{\lambda} \frac{\partial}{\partial \chi} \delta b\left(\lambda b_{0}, \lambda b_{0}^{\prime}, \ldots\right)\right] .
\end{aligned}
$$


Integrating the latter equation over $v^{2}$, we obtain

$$
\begin{aligned}
-m \frac{\partial}{\partial m} b\left(v^{2}, u^{2}\right)= & \epsilon b\left(v^{2}, u^{2}\right)+\frac{1}{2} \partial_{v^{2}} b\left(v^{2}, u^{2}\right)^{2}-\partial_{v^{2}} b\left(v^{2}, u^{2}\right) \partial_{v^{2}} b(0,0)+\left.\epsilon \frac{\partial}{\partial \lambda}\right|_{\lambda=1}\left[\frac{1}{\lambda} \delta b\left(\lambda b_{0}^{\prime}, \lambda b_{0}^{\prime \prime}, \ldots\right)\right] \\
& -\left.\partial_{v^{2}} b\left(v^{2}, u^{2}\right) \lim _{v, u \rightarrow 0} \frac{\partial}{\partial \lambda}\right|_{\lambda=1}\left[\frac{1}{\lambda} \frac{\partial}{\partial \chi} \delta b\left(\lambda b_{0}, \lambda b_{0}^{\prime}, \ldots\right)\right]
\end{aligned}
$$

which of course has to be read at $u^{2}=v^{2}$. In a final step, we want to reintroduce proper quantities. Noting that

$$
\left.\partial_{v^{2}} b\left(v^{2}, u^{2}\right)\right|_{u=v^{2}}=b^{\prime}\left(v^{2}\right)-\partial_{v^{2}} \delta b\left(\chi(v), v^{2}\right)
$$

(which does not need $u$ ), we obtain

$$
\begin{aligned}
-m \frac{\partial}{\partial m} b\left(v^{2}\right)= & \epsilon b\left(v^{2}\right)+\frac{1}{2} b^{\prime}\left(v^{2}\right)^{2}-b^{\prime}\left(v^{2}\right) b^{\prime}(0)-\left[b^{\prime}\left(v^{2}\right)-b^{\prime}(0)\right] \partial_{v^{2}} \delta b\left(\chi(v), v^{2}\right) \\
& +\partial_{v^{2}} \delta b(\chi(0), 0) b^{\prime}\left(v^{2}\right)+\frac{1}{2}\left[\partial_{v^{2}} \delta b\left(\chi(v), v^{2}\right)\right]^{2}-\partial_{v^{2}} \delta b\left(\chi(v), v^{2}\right) \partial_{v^{2}} \delta b(\chi(0), 0) \\
& +\left.\epsilon \frac{\partial}{\partial \lambda}\right|_{\lambda=1}\left[\frac{1}{\lambda} \delta b\left(\lambda b_{0}^{\prime}, \lambda b_{0}^{\prime \prime}, \ldots\right)\right] \\
& -\left.\left[b^{\prime}\left(v^{2}\right)-\partial_{v^{2}} b\left(\chi(v), v^{2}\right)\right] \lim _{v, u \rightarrow 0} \frac{\partial}{\partial \lambda}\right|_{\lambda=1}\left[\frac{1}{\lambda} \frac{\partial}{\partial \chi} \delta b\left(\lambda b_{0}, \lambda b_{0}^{\prime}, \ldots\right)\right]
\end{aligned}
$$

(Of course $\partial_{v^{2}} \delta b(\chi(0), 0)$ means first to derive and then to put the arguments to 0 .) Also note that $\partial_{v^{2}} \delta b(\chi(0), 0)$ is not 0 , at least at order $T$. The $\beta$-function at order $1 / N$ therefore is

$$
\begin{aligned}
-m \frac{\partial}{\partial m} b\left(v^{2}\right)= & \epsilon b\left(v^{2}\right)+\frac{1}{2} b^{\prime}\left(v^{2}\right)^{2}-b^{\prime}\left(v^{2}\right) b^{\prime}(0) \\
+ & +\frac{1}{N}\left(\left.\epsilon \frac{\partial}{\partial \lambda}\right|_{\lambda=1}\left[\frac{1}{\lambda} b^{(1)}\left(\lambda b_{0}^{\prime}, \lambda b_{0}^{\prime \prime}, \ldots\right)\right]-\left[b^{\prime}\left(v^{2}\right)-b^{\prime}(0)\right] \partial_{v^{2}} b^{(1)}\left(\chi(v), v^{2}\right)\right. \\
& \left.\quad+b^{\prime}\left(v^{2}\right)\left\{\left.\partial_{u^{2}} b^{(1)}\left(\chi(0), u^{2}\right)\right|_{u=0}-\left.\lim _{v, u \rightarrow 0} \frac{\partial}{\partial \lambda}\right|_{\lambda=1}\left[\frac{1}{\lambda} \frac{\partial}{\partial \chi} b^{(1)}\left(\lambda b_{0}, \lambda b_{0}^{\prime}, \ldots\right)\right]\right\}\right) \\
+ & O\left(\frac{1}{N^{2}}\right)
\end{aligned}
$$

This might better be grouped as

$$
\begin{aligned}
-m \frac{\partial}{\partial m} b\left(v^{2}\right)= & \epsilon b\left(v^{2}\right)+\frac{1}{2} b^{\prime}\left(v^{2}\right)^{2}-b^{\prime}\left(v^{2}\right) b^{\prime}(0) \\
& +\frac{1}{N}\left(\left.\epsilon \frac{\partial}{\partial \lambda}\right|_{\lambda=1}\left[\frac{1}{\lambda} b^{(1)}\left(\lambda b_{0}^{\prime}, \lambda b_{0}^{\prime \prime}, \ldots\right)\right]-\left.b^{\prime}\left(v^{2}\right) \lim _{v, u \rightarrow 0} \frac{\partial}{\partial \lambda}\right|_{\lambda=1}\left[\frac{1}{\lambda} \frac{\partial}{\partial \chi} b^{(1)}\left(\lambda b_{0}, \lambda b_{0}^{\prime}, \ldots\right)\right]\right. \\
& \left.\quad-\left[b^{\prime}\left(v^{2}\right)-b^{\prime}(0)\right] \partial_{v^{2}} b^{(1)}\left(\chi(v), v^{2}\right)+\left.b^{\prime}\left(v^{2}\right) \partial_{u^{2}} b^{(1)}\left(\chi(0), u^{2}\right)\right|_{u=0}\right) \\
+ & O\left(\frac{1}{N^{2}}\right) .
\end{aligned}
$$

A caveat is in order: The rescaling has to be done on the level of bare vertices, not on the level of renormalized ones. That would give a wrong result. However the derivative w.r.t. $v^{2}$ can be taken in any formulation. 


\section{G.3 The case $d=0$}

As one can see from our final result for the $\beta$-function in (7.7), specified to $d=0$, it is a polynom in $b$ of finite order, since the denominators present in $h_{x}(p)$, see (6.13) are identical 1. Since this come as quite a surprise, we show here why this must be so; actually it is a quite general feature of the $1 / N$-expansion of a renormalizable theory in $d=0$.

We start to warm up with the diagram

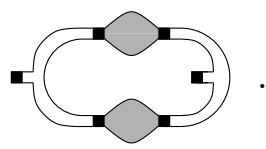

The leading and next-to-leading contributions (in $b$ ) are
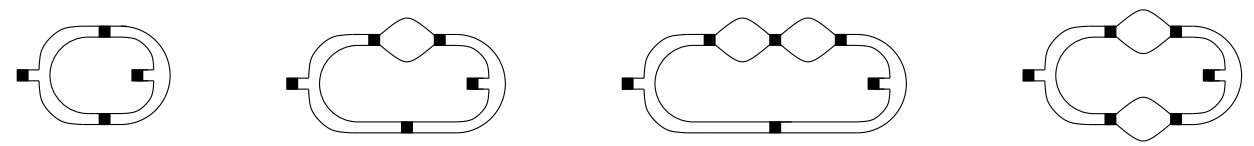

Our strategy is to apply Bogoliubov's $\mathcal{R}$-operation, see e.g. [67-70] and to show that only the first two terms contribute. Three remarks are in order:

1.) The $\mathcal{R}$-operation in the context of a $1 / N$-expansion is maybe not entirely natural. However we have in the above diagrams the property, that the terms already encountered at $1 / N$ (and thus taken care of in the $\beta$-function at leading order) are exactly the iterated 1-loop diagrams, thus the first order in $1 / N$.

2.) In order to extract the $\beta$-function from $\mathcal{R}$ applied to a diagram, we only have to derive (w.r.t. $m$ ) the diagrams in the boxes, since only those are counter-terms.

3.) Applying $\mathcal{R}$ to a functional of the bare $b_{0}$ gives the result as a functional of $b$. Thus the contribution $\delta \beta(b)$ to the $\beta$-function is $\delta \beta(b)=-m \frac{\partial}{\partial m} \mathcal{R}\left[\operatorname{diagram}\left(b_{0}\right)\right]$. Now

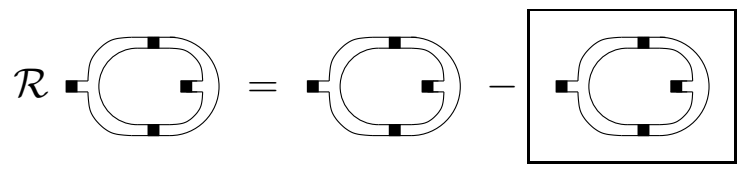

which give as the contribution to $\beta$

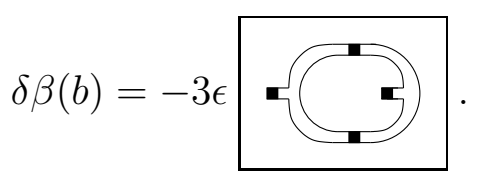

The second diagram gives

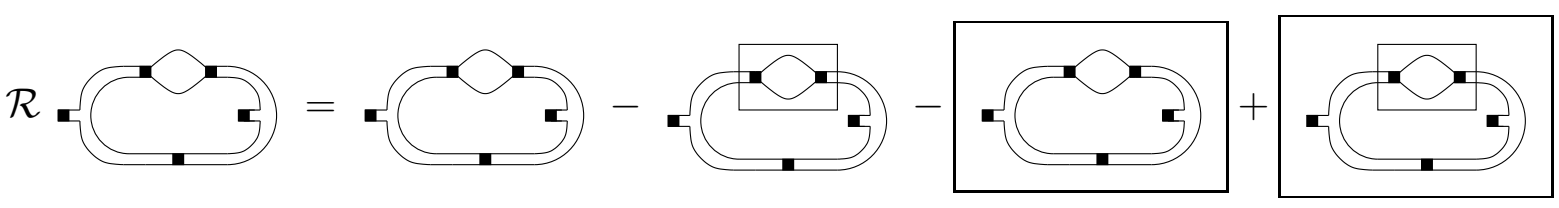

In $D=0$, the last two diagrams cancel, and

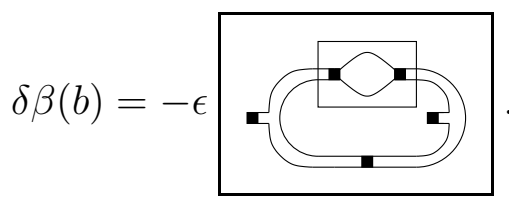


This gives also the ratio 6 to be found in the explicit formula: The 3 from the first diagram has to be set in relation to the combinatorial factor of 2 for the second one and a factor of $1 / \varepsilon$ for the second, which together give a ratio of 6 ; finishing the test.

We now proceed to higher orders: First we remark that for the chain with $n$ members (here $n=3$ ) one can show recursively that

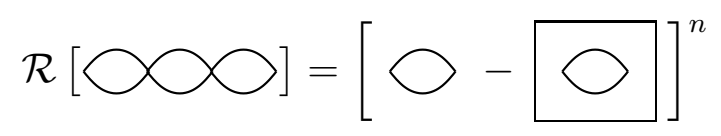

Thus having $n$ diagrams in the chain and deriving the $n$-dependence gives

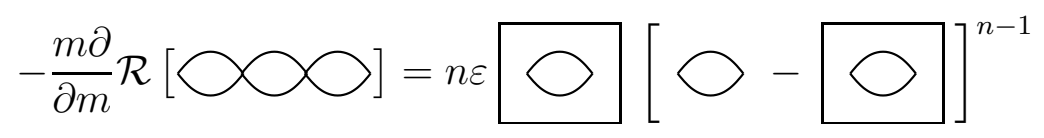

Of course, in $d=0$ the latter vanishes for $n>1$.

It is now easy to see that only finite order terms can appear, q.e.d.

\section{H Details of the calculation of the $\beta$-function at finite $T$}

\section{H.1 Integrals appearing in the $T>0$ correction to the effective action}

The following functions have been defined but not given in formula (6.17) in the main text.

$$
\begin{aligned}
g_{4}\left(a_{x}\right) & =\frac{1}{A_{d}} \int_{p} c(p) h_{x}(p)+\frac{1}{2} c(p) i_{2}(p) h_{x}(p)^{2} \\
g_{5}\left(a_{x}\right) & =-\frac{1}{2} \frac{1}{A_{d}} \int_{p} c(p) i_{2}(p)^{2} h_{x}(p)^{2} \\
g_{6}\left(a_{x}\right) & =\frac{1}{A_{d}} \int_{p} i_{3}(p) h_{x}(p)+\frac{1}{2} i_{2}(p) i_{3}(p) h_{x}(p)^{2} \\
g_{7}\left(a_{x}\right) & =-\frac{1}{2} \frac{1}{A_{d}} \int_{p} c(p) i_{2}(p)^{2} i_{3}(p) h_{x}(p)^{2} \\
g_{8}\left(a_{0}\right) & =-\frac{1}{A_{d}} \int_{p} i_{3}(p) h_{0}(p) \\
g_{9}\left(a_{0}\right) & =-\frac{1}{2} \frac{1}{A_{d}} \int_{p} i_{2}(p) i_{3}(p) h_{0}(p) \\
g_{10}\left(a_{x}\right) & =\frac{1}{8 A_{d}} \int_{p}-\ln \left(1-a_{x} i_{2}(p)\right)+i_{2}(p) h_{x}(p)+\frac{1}{4} i_{2}(p)^{2} h_{x}(p)^{2} \\
g_{11}\left(a_{x}\right) & =-\frac{1}{16 A_{d}} \int_{p} i_{2}(p)^{2} h_{x}(p)+i_{2}(p)^{3} h_{x}(p)^{2} \\
g_{12}\left(a_{x}\right) & =\frac{1}{16 A_{d}} \int_{p}-i_{2}(p)^{3} h_{x}(p)+\frac{1}{2} i_{2}(p)^{4} h_{x}(p)^{2} \\
\gamma & =-\frac{1}{4} i_{1}^{2} i_{2} \\
g_{13}\left(a_{0}\right) & =-\frac{3}{8} i_{2} \int i_{2}(p)^{2} h_{0}(p)
\end{aligned}
$$


and we recall that a cutoff $\Lambda / m$ is implicit in all these rescaled momentum integrals.

These functions are not all independent. Indeed, defining

$$
\gamma_{m, n}\left(a_{x}\right):=\frac{1}{A_{d}} \int_{p} f(p) i_{2}(p)^{n} h_{x}(p)^{m}
$$

one easily establishes the recursion relations:

$$
\begin{aligned}
m \gamma_{m+1, n}(a) & =a^{2} \partial_{a} \gamma_{m n}(a) \\
\gamma_{m+1, n+1}(a) & =\frac{1}{a} \gamma_{m+1, n}(a)-\gamma_{m, n}(a)
\end{aligned}
$$

They allow to obtain these functions from derivatives of

$$
\gamma(a)=-\frac{1}{A_{d}} \int_{p} \ln \left(1-a i_{2}(p)\right) f(p)
$$

Specializing first to $f(p)=1$, one finds using (H.13)

$$
\begin{aligned}
\gamma_{1,1}(a) & =a \gamma^{\prime}(a) \\
\gamma_{1,2}(a) & =\gamma^{\prime}(a)-i_{1}^{2} \\
\gamma_{1,3}(a) & =\frac{1}{a}\left(\gamma^{\prime}(a)-i_{1}^{2}\right)-\gamma_{0,2}(a) \\
\gamma_{2,2}(a) & =a^{2} \gamma^{\prime \prime}(a) \\
\gamma_{2,3}(a) & =a \gamma^{\prime \prime}(a)-\gamma^{\prime}(a)+i_{1}^{2} \\
\gamma_{2,4}(a) & =\gamma^{\prime \prime}(a)-\frac{2}{a}\left(\gamma^{\prime}(a)-i_{1}^{2}\right)+\gamma_{0,2}(a)
\end{aligned}
$$

Thus the functions $g_{10}(a), g_{11}(a), g_{12}(a)$ and $g_{13}(a)$ can all be obtained from derivatives of $\gamma(a)$ with $f(p)=1$. Similarly, $g_{4}(a)$ and $g_{5}(a)$ can be all obtained from derivatives of $\gamma(a)$ with $f(p)=c(p)$, and similarly for $g_{6}(a), g_{8}(a)$ and $g_{9}(a)$ with $f(p)=i_{3}(p), g_{7}(a)$ with $f(p)=i_{3}(p) c(p)$.

One may further attempt to relate $\gamma(a)$ for different functions $f(p)$. Since $\partial_{m^{2}} I_{2}=-2 I_{3}$, one can use that

$$
i_{3}(p)=\frac{1}{4} \epsilon i_{2}(p)-\frac{1}{4} p i_{2}^{\prime}(p)-\frac{1}{4} \lambda \partial_{\lambda} i_{2}(p)
$$

where $\lambda=\Lambda / m$. Integration by part yields identities such as

$$
\int \frac{\mathrm{d} p}{p} p^{d}\left(p \partial_{p}+\lambda \partial_{\lambda}\right) H\left(i_{2}(p)\right)=-d \int^{\lambda} \frac{\mathrm{d} p}{p} p^{d} H\left(i_{2}(p)\right)+\lambda \partial_{\lambda} \int^{\lambda} \frac{\mathrm{d} p}{p} p^{d} H\left(i_{2}(p)\right),
$$

which can be used to relate the integrals.

\section{H.2 Calculation of the $T>0 \beta$-function}

Below we compute $-m \partial_{m} b(x)$ at fixed $\Lambda / m$, thus we truly compute $-\left(m \partial_{m}+\Lambda \partial_{\Lambda}\right) b(x)$. It is therefore useful at $T>0$ only for $d<2$ when all integrals are UV convergent and the limit $\Lambda / m \rightarrow \infty$ can be taken with no further redefinitions. The calculation of the $\beta$-function for $d \geq 2$ and $T>0$ requires further redefinitions and will eventually be detailed elsewhere. 
Taking into account all $m$-dependence at $T>0$ in (6.17) one obtains (up to an additive constant):

$$
\begin{aligned}
& \beta_{1}[b](x)=-m \partial_{m} b(x) \\
& =\frac{\delta b_{1}}{\delta a_{x}}\left[-m \partial_{m}^{0} a_{x}\right]+\frac{\delta b_{1}}{\delta\left[b^{\prime}(x)-b^{\prime}(0)\right]}\left[-m \partial_{m}^{0}\left(b^{\prime}(x)-b^{\prime}(0)\right)\right]+\frac{\delta b_{1}}{\delta a_{0}}\left(-m \partial_{m}^{0} a_{0}\right)+\frac{\delta b_{1}}{\delta T_{m}}\left(-m \partial_{m}^{0} T_{m}\right) \\
& \quad+\frac{\delta b_{1}}{\delta \alpha}\left(-m \partial_{m} \alpha\right)-\epsilon b_{1}-\left(b^{\prime}-b^{\prime}(0)\right)\left(b_{1}^{\prime}-b_{1}^{\prime}(0)\right)-T_{m} \frac{b_{1}^{\prime}(x)}{1+b^{\prime \prime}(0) / \epsilon}+T_{m} \frac{b^{\prime}(x)}{\left(1+b^{\prime \prime}(0) / \epsilon\right)^{2}} \frac{b_{1}^{\prime \prime}(0)}{\epsilon}
\end{aligned}
$$

where $b_{1}$ is given in (6.17).

One uses that:

$$
\begin{aligned}
-m \partial_{m}^{0} a_{x} & =\epsilon a_{x}+\left[b^{\prime}(x)-b^{\prime}(0)\right] a_{x}^{\prime}+\frac{T_{m}}{1+b^{\prime \prime}(0) / \epsilon} a_{x}^{\prime} \\
-m \partial_{m}^{0}\left(b^{\prime}(x)-b^{\prime}(0)\right) & =\left[\epsilon+b^{\prime \prime}(x)\right]\left[b^{\prime}(x)-b^{\prime}(0)\right]+\frac{T_{m}}{1+b^{\prime \prime}(0) / \epsilon}\left[b^{\prime \prime}(x)-b^{\prime \prime}(0)\right] \\
-m \partial_{m}^{0} \alpha & =\alpha \epsilon+T_{m}\left(\frac{\alpha^{2}}{a_{0}-\epsilon}+\frac{\left(a_{0}-\epsilon\right)^{2} \bar{\alpha}}{\epsilon}\right)
\end{aligned}
$$

and $-m \partial_{m} T_{m}=-\theta T_{m}$. After a rather tedious calculation one obtains the form (7.13) given in the text with the following definitions (for $d<2$ ):

$$
\begin{aligned}
\tilde{g}_{4}(a)= & -4 g_{1}(a)-\epsilon(\epsilon+\theta) g_{4}(a)+\epsilon^{2} a g_{4}^{\prime}(a) \\
\tilde{g}_{5}(a)= & \frac{4}{\epsilon} g_{1}(a)-2 g_{2}(a)-\epsilon \theta g_{5}(a)+\epsilon^{2} a g_{5}^{\prime}(a) \\
\tilde{g}_{6}(a)= & -2 g_{2}(a)-\epsilon \theta g_{6}(a)+\epsilon^{2} a g_{6}^{\prime}(a)-\epsilon g_{4}(a) \\
\tilde{g}_{7}(a)= & \frac{2}{\epsilon} g_{2}(a)-4 g_{3}(a)-\epsilon g_{5}(a)+\epsilon(\epsilon-\theta) g_{7}(a)+\epsilon^{2} a g_{7}^{\prime}(a) \\
\tilde{g}_{8}(a)= & \frac{a \epsilon^{2}}{\epsilon-a}\left[g_{6}(a)+g_{8}(a)+a\left(g_{7}(a)+g_{9}(a)\right)\right]+\frac{4}{\epsilon} g_{1}(a)+\epsilon g_{4}(a)-\epsilon \theta g_{8}(a) \\
& +a\left[-\frac{8}{\epsilon^{2}} g_{1}(a)+\frac{4}{\epsilon} g_{2}(a)+\epsilon g_{5}(a)+\epsilon(\epsilon-\theta) g_{9}(a)+\epsilon^{2} g_{8}^{\prime}(a)\right] \\
& +a^{2}\left[\frac{4}{\epsilon^{3}} g_{1}(a)-\frac{4}{\epsilon^{2}} g_{2}(a)+\frac{4}{\epsilon} g_{3}(a)+\epsilon^{2} g_{9}^{\prime}(a)\right] \\
\tilde{g}_{10}(a)= & -\epsilon^{2}(\epsilon+2 \theta) g_{10}(a)-\epsilon g_{4}(a)+\epsilon^{3} a g_{10}^{\prime}(a) \\
\tilde{g}_{11}(a)= & -2 \epsilon^{2} \theta g_{11}(a)+g_{4}(a)-\epsilon g_{5}(a)-\epsilon g_{6}(a)+\epsilon^{3} a g_{11}^{\prime}(a) \\
\tilde{g}_{12}(a)= & \epsilon^{2}(\epsilon-2 \theta) g_{12}(a)+g_{5}(a)-\epsilon g_{7}(a)+\epsilon^{3} a g_{12}^{\prime}(a) \\
\phi(a)= & \frac{\epsilon}{\epsilon-a}\left[g_{6}(a)+a g_{7}(a)+g_{8}(a)+\epsilon g_{9}(a)+\epsilon^{2} g_{10}^{\prime}(a)+a \epsilon^{2} g_{11}^{\prime}(a)+a^{2} \epsilon^{2} g_{12}^{\prime}(a)+\epsilon^{3} \gamma+a \epsilon^{3} g_{13}(a)\right] \\
& +2 g_{4}^{\prime}(a)+\epsilon g_{8}^{\prime}(a)-2 \epsilon^{2} \gamma \theta+a\left[\epsilon^{2}(\epsilon-2 \theta) g_{13}(a)-\frac{2}{\epsilon} g_{4}^{\prime}(a)+2 g_{5}^{\prime}(a)+2 g_{6}^{\prime}(a)+\epsilon g_{9}^{\prime}(a)\right] \\
& +a^{2}\left[\epsilon^{3} g_{13}^{\prime}(a)-\frac{2 g_{5}^{\prime}(a)}{\epsilon}+2 g_{7}^{\prime}(a)\right] \\
\tilde{\psi}(a)= & \epsilon\left[g_{10}^{\prime \prime}(a)+a g_{11}^{\prime \prime}(a)+a^{2} g_{12}^{\prime \prime}(a)+\epsilon g_{13}(a)+a \epsilon g_{13}^{\prime}(a)\right]
\end{aligned}
$$




\section{Integrals}

\section{I.1 Definitions}

$$
\begin{aligned}
I_{n} & :=\int_{k} \frac{1}{\left(k^{2}+m^{2}\right)^{n}} \\
I_{2} & =A_{d} \frac{m^{-\epsilon}}{\epsilon} \\
A_{d} & :=\frac{2 \Gamma(3-d / 2)}{(4 \pi)^{d / 2}} .
\end{aligned}
$$

The momentum dependent ones are

$$
\begin{aligned}
I_{2}(p) & :=\int_{k} \frac{1}{(k+p / 2)^{2}+m^{2}} \frac{1}{(k-p / 2)^{2}+m^{2}} \\
I_{3}(p) & :=\int_{k} \frac{1}{\left[(k+p / 2)^{2}+m^{2}\right]^{2}} \frac{1}{(k-p / 2)^{2}+m^{2}} \\
I_{4}(p) & :=\int_{k} \frac{1}{\left[(k+p / 2)^{2}+m^{2}\right]^{2}} \frac{1}{\left[(k-p / 2)^{2}+m^{2}\right]^{2}} .
\end{aligned}
$$

Dimensionless rescaled variants:

$$
\begin{aligned}
i_{n}(p) & :=\left.\frac{I_{n}(p)}{A_{d}}\right|_{m=1} \\
i_{n} & :=\left.\frac{I_{n}}{A_{d}}\right|_{m=1} .
\end{aligned}
$$

\section{I.2 Integrals in fixed dimensions, general formulas}

The general case can be treated as follows:

$$
j_{n, m}(p):=\frac{1}{A_{d}} \int_{k} \frac{1}{\left[\left(k-\frac{p}{2}\right)^{2}+1\right]^{m}} \frac{1}{\left[\left(k+\frac{p}{2}\right)^{2}+1\right]^{n}} .
$$

Using the usual Schwinger-parameter representation, this can be written as

$$
\begin{aligned}
j_{n, m}(p) & =\frac{1}{\Gamma(n) \Gamma(m)}\left(\frac{1}{A_{d}} \int_{k} \mathrm{e}^{-k^{2}}\right) \int_{\alpha, \beta>0} \alpha^{n-1} \beta^{m-1}(\alpha+\beta)^{-\frac{d}{2}} \mathrm{e}^{-(\alpha+\beta)} \mathrm{e}^{-\frac{\alpha \beta}{\alpha+\beta} p^{2}} \\
& =\frac{\Gamma(n+m-d / 2)}{2 \Gamma(3-d / 2) \Gamma(n) \Gamma(m)} \int_{\beta>0} \frac{\beta^{m-1}}{(1+\beta)^{n+m}}\left[1+\frac{\beta}{(1+\beta)^{2}} p^{2}\right]^{\frac{d}{2}-n-m} .
\end{aligned}
$$

We make the change of variables $\beta=\frac{s}{1-s}$ :

$$
\begin{aligned}
j_{n, m}(p)= & \frac{\Gamma(n+m-d / 2)}{2 \Gamma(3-d / 2) \Gamma(n) \Gamma(m)} \int_{0}^{1} \mathrm{~d} s s^{m-1}(1-s)^{n-1}\left[1+s(1-s) p^{2}\right]^{\frac{d}{2}-m-n} \\
= & \frac{\Gamma(n+m-d / 2)}{\Gamma(3-d / 2) \Gamma(n) \Gamma(m) 2^{n+m}} \\
& \times \int_{0}^{1} \frac{\mathrm{d} y}{\sqrt{1-y}} \frac{(1+\sqrt{1-y})^{m-1}(1-\sqrt{1-y})^{n-1}+(1+\sqrt{1-y})^{n-1}(1-\sqrt{1-y})^{m-1}}{2}\left[1+y \frac{p^{2}}{4}\right]^{\frac{d}{2}-m-n},
\end{aligned}
$$


where we have used another new variable $y=4 s(1-s)$. Note that the large fraction $\frac{\cdots}{2}$ in the above expression is such that only integer powers of $(1-y)$ survive. Some simplifications occur for $n=m$, and $i_{3}(p)=j_{1,2}(p)$ :

$$
\begin{aligned}
j_{n, n}(p) & =\frac{\Gamma(2 n-d / 2)}{\Gamma(3-d / 2) \Gamma(n)^{2} 2^{2 n}} \int_{0}^{1} \frac{\mathrm{d} y}{\sqrt{1-y}} y^{n-1}\left[1+y \frac{p^{2}}{4}\right]^{\frac{d}{2}-2 n} \\
i_{3}(p)=j_{1,2}(p) & =\frac{1}{8} \int_{0}^{1} \frac{\mathrm{d} y}{\sqrt{1-y}}\left[1+y \frac{p^{2}}{4}\right]^{\frac{d}{2}-3} .
\end{aligned}
$$

I.3 $d=0$

$$
i_{n}=i_{n}(p)=1 / 4, \quad A_{d}=4, \quad \epsilon=4
$$

I.4 $d=1$

$$
\begin{aligned}
A_{d} & =\frac{3}{4} \\
i_{1} & =\frac{2}{3} \\
i_{2}(p) & =\frac{4}{3\left(4+p^{2}\right)} \\
i_{3}(p) & =\frac{12+p^{2}}{3\left(4+p^{2}\right)^{2}} \\
i_{4}(p) & =\frac{2\left(20+p^{2}\right)}{3\left(4+p^{2}\right)^{3}} .
\end{aligned}
$$

$$
\begin{gathered}
\text { I.5 } \quad d=2 \\
A_{d}=\frac{1}{2 \pi}
\end{gathered}
$$

$$
\begin{gathered}
i_{2}(p)=\frac{\operatorname{arctanh}\left(\frac{|p| \sqrt{4+p^{2}}}{2+p^{2}}\right)}{|p| \sqrt{4+p^{2}}}=\frac{\ln \left(2+p^{2}+|p| \sqrt{4+p^{2}}\right)-\ln \left(2+p^{2}-|p| \sqrt{4+p^{2}}\right)}{2|p| \sqrt{4+p^{2}}}=\frac{2 \operatorname{arcsinh}\left(\frac{|p|}{2}\right)}{|p| \sqrt{4+p^{2}}} \\
i_{3}(p)=\frac{1}{8+2 p^{2}}+\frac{2 \operatorname{arcsinh}\left(\frac{|p|}{2}\right)}{|p|\left(4+p^{2}\right)^{\frac{3}{2}}} \\
i_{4}(p)=\frac{1}{6}{ }_{2} F_{1}\left(2,3,5 / 2,-p^{2} / 4\right)
\end{gathered}
$$

$$
\begin{gathered}
\text { I.6 } \quad d=3 \\
A_{d}=\frac{1}{8 \pi} \\
i_{2}(p)=2 \frac{\arctan \left(\frac{|p|}{2}\right)}{|p|}=\frac{i}{|p|}[\ln (2-i|p|)-\ln (2+i|p|)]
\end{gathered}
$$




$$
\begin{gathered}
i_{3}(p)=\frac{1}{p^{2}+4} \\
i_{4}(p)=\frac{2}{\left(p^{2}+4\right)^{2}} .
\end{gathered}
$$




\section{J Summary of Notation}

\begin{tabular}{|c|c|c|}
\hline symbol & definition & defined in equation \\
\hline$\epsilon$ & $\epsilon=4-d$ & \\
\hline$\zeta, \theta$ & $\zeta=$ roughness, $\theta=d-2+2 \zeta$ (thermal exponent) & \\
\hline$u(x), v(x)$ & $u(x)=$ field, $v(x)=u(x) / \sqrt{N}$ & \\
\hline$I_{n}$ & $I_{n}:=\int_{k} \frac{1}{\left(k^{2}+m^{2}\right)^{n}}, I_{2}=A_{d} \frac{m^{-\epsilon}}{\epsilon}, A_{d}=\frac{2 \Gamma(3-d / 2)}{(4 \pi)^{d / 2}}$ & $(2.14)$ \\
\hline$I_{2}(p)$ & $I_{2}(p):=\int_{k} \frac{1}{(k+p / 2)^{2}+m^{2}} \frac{1}{(k-p / 2)^{2}+m^{2}}$ & $(4.18)$ \\
\hline$I_{3}(p)$ & $I_{3}(p):=\int_{k} \frac{1}{\left[(k+p / 2)^{2}+m^{2}\right]^{2}} \frac{1}{(k-p / 2)^{2}+m^{2}}$ & $(4.20)$ \\
\hline$I_{4}(p)$ & $I_{4}(p):=\int_{k} \frac{1}{\left[(k+p / 2)^{2}+m^{2}\right]^{2}} \frac{1}{\left[(k-p / 2)^{2}+m^{2}\right]^{2}}$ & $(4.21)$ \\
\hline$C(p)$ & $C(p):=\left(p^{2}+m^{2}\right)^{-1}$ & \\
\hline$i_{n}(p), i_{n}$ & $i_{n}(p):=\left.\frac{I_{n}(p)}{A_{d}}\right|_{m=1}, i_{n}:=\left.\frac{I_{n}}{A_{d}}\right|_{m=1}$ & $(6.15),(6.16)$ \\
\hline$c(p)$ & $c(p):=\left(1+p^{2}\right)^{-1}$ & \\
\hline$B(\ldots)$ & second cumulant of bare disorder & \\
\hline$\tilde{B}(\ldots)$ & second cumulant of renormalized disorder (not rescaled) & \\
\hline$B_{a b}^{\prime}, B_{a b}^{\prime \prime}, \tilde{B}_{a b}^{\prime}$, etc. & $B_{a b}^{\prime}:=B^{\prime}\left(\bar{\chi}_{a b}\right), B_{a b}^{\prime \prime}:=B^{\prime \prime}\left(\bar{\chi}_{a b}\right), \tilde{B}_{a b}^{\prime}:=\tilde{B}^{\prime}\left(v_{a b}^{2}\right)$, etc. & \\
\hline$b(z)$ & $b(z):=4 A_{d} m^{4 \zeta-\epsilon} \tilde{B}\left(z m^{-2 \zeta}\right)$ & $(6.10)$ \\
\hline$\chi_{a b}(x), \lambda_{a b}(x)$ & auxiliary fields & \\
\hline$\tilde{\chi}_{a b}(x)$ & $\tilde{\chi}_{a b}(x):=\chi_{a b}(x)+\chi_{b a}(x)-\chi_{a a}(x)-\chi_{b b}(x)$ & \\
\hline$\tilde{\chi}_{v}^{a b}, \bar{\chi}_{v}^{a b}$ & $\tilde{\chi}_{v}^{a b}=\left.\tilde{\chi}_{a b}(x)\right|_{v(x)=v}, \quad \tilde{\chi}_{v}^{a b}=\bar{\chi}_{v}^{a b}+O\left(\frac{1}{N}\right)$ & \\
\hline $\bar{\chi}_{v}^{a b}=\bar{\chi}_{v}=\bar{\chi}_{a b}$ & $\bar{\chi}_{v}^{a b}:=v_{a b}^{2}+2 T I_{1}+4 I_{2}\left[\tilde{B}_{a b}^{\prime}-\frac{1}{2}\left(\tilde{B}_{a a}^{\prime}+\tilde{B}_{b b}^{\prime}\right)\right]$ & \\
\hline$H_{v}(p)$ & $H_{v}(p):=\frac{B^{\prime \prime}\left(\bar{\chi}_{v}\right)}{1-4 I_{2}(p) B^{\prime \prime}\left(\bar{\chi}_{v}\right)}$ & $(4.27)$ \\
\hline$\tilde{H}_{x}(p)$ & $\tilde{H}_{x}(p):=\frac{\tilde{B}^{\prime \prime}(x)}{1+4\left[I_{2}-I_{2}(p)\right] \tilde{B}^{\prime \prime}(x)}$ & $(6.8)$ \\
\hline$h_{x}(p)$ & $h_{x}(p):=\frac{b^{\prime \prime}(x)}{1+\left[i_{2}-i_{2}(p)\right] b^{\prime \prime}(x)}$ & $(6.13)$ \\
\hline$T$ & temperature & \\
\hline$T_{m}$ & $T_{m}:=4 T A_{d} m^{\theta} / \epsilon$ & \\
\hline
\end{tabular}




\section{References}

[1] P. Chauve, P. Le Doussal and K.J. Wiese, Renormalization of pinned elastic systems: How does it work beyond one loop?, Phys. Rev. Lett. 86 (2001) 1785-1788, cond-mat/0006056.

[2] P. Le Doussal and K.J. Wiese, Functional renormalization group at large $N$ for random manifolds, cond-mat/0109204 (2001).

[3] P. Le Doussal and K.J. Wiese, Functional renormalization group at large $N$ for random manifolds, Phys. Rev. Lett. 89 (2002) 125702, cond-mat/0109204v1.

[4] P. Le Doussal, K.J. Wiese and P. Chauve, 2-loop functional renormalization group analysis of the depinning transition, Phys. Rev. B 66 (2002) 174201, cond-mat/0205108.

[5] P. Le Doussal and K.J. Wiese, Functional renormalization group for anisotropic depinning and relation to branching processes, Phys. Rev. E 67 (2003) 016121, cond-mat/0208204.

[6] P. Le Doussal and K.J. Wiese, Higher correlations, universal distributions and finite size scaling in the field theory of depinning, Phys. Rev. E 68 (2003) 046118, cond-mat/0301465.

[7] P. Le Doussal, K.J. Wiese and P. Chauve, Functional renormalization group and the field theory of disordered elastic systems, cont-mat/0304614 (2003).

[8] P. Le Doussal and K.J. Wiese, Functional renormalization group at large $N$ for disordered elastic systems, and relation to replica symmetry breaking, Phys. Rev. B 68 (2003) 17402, cond-mat/0305634.

[9] A. Rosso, W. Krauth, P. Le Doussal, J. Vannimenus and K.J. Wiese, Universal interface width distributions at the depinning threshold, Phys. Rev. E 68 (2003) 036128, cond-mat/0301464.

[10] P. Chauve and P. Le Doussal, Exact multilocal renormalization group and applications to disordered problems, Phys. Rev. E 64 (2001) 051102/1-27, cond-mat/9602023.

[11] M. Mézard and G. Parisi, Replica field theory for random manifolds, J. Phys. I (France) 1 (1991) 809-837.

[12] M. Mezard and G. Parisi, Manifolds in random media: two extreme cases, J. Phys. I (France) 2 (1992) 2231-42.

[13] E. Brunet and B. Derrida, Probability distribution of the free energy of a directed polymer in a random medium, Phys. Rev. E 61 (2000) 6789-801.

[14] E. Brunet and B. Derrida, Ground state energy of a non-integer number of particles with delta attractive interactions, Physica A 279 (2000) 398-407.

[15] D.S. Fisher, Sliding charge-density waves as a dynamical critical phenomena, Phys. Rev. B 31 (1985) 1396-1427.

[16] DS. Fisher, Random fields, random anisotropies, nonlinear sigma models and dimensional reduction, Phys. Rev. B 31 (1985) 7233-51.

[17] D.S. Fisher, Interface fluctuations in disordered systems: 5 - $\epsilon$ expansion, Phys. Rev. Lett. 56 (1986) 1964-97. 
[18] T. Nattermann, Interface roughening in systems with quenched random impurities, Europhys. Lett. 4 (1987) 1241-6.

[19] O. Narayan and D.S. Fisher, Logarithmic effects on the critical behavior of superfluids in random media, Phys. Rev. B 42 (1990) 7869-75.

[20] T. Nattermann, S. Stepanow, L.H. Tang and H. Leschhorn, Dynamics of interface depinning in a disordered medium, J. Phys. II (France) 2 (1992) 1483-1488.

[21] O. Narayan and D.S. Fisher, Dynamics of sliding charge-density waves in 4- epsilon dimensions, Phys. Rev. Lett. 68 (1992) 3615-18.

[22] O. Narayan and D.S. Fisher, Critical behavior of sliding charge-density waves in 4- epsilon dimensions, Phys. Rev. B 46 (1992) 11520-49.

[23] O. Narayan and D.S. Fisher, Threshold critical dynamics of driven interfaces in random media, Phys. Rev. B 48 (1993) 7030-42.

[24] O. Narayan and D.S. Fisher, Nonlinear fluid flow in random media: critical phenomena near threshold, Phys. Rev. B 49 (1993) 9469-502.

[25] G. Blatter, M.V. Feigel'man, V.B. Geshkenbein, A.I. Larkin and V.M. Vinokur, Vortices in hightemperature superconductors, Rev. Mod. Phys. 66 (1994) 1125.

[26] D. Ertas and M. Kardar, Anisotropic scaling in depinning of a flux line, Phys. Rev. Lett. 73 (1994) 1703-6.

[27] D. Ertas and M. Kardar, Anisotropic scaling in threshold critical dynamics of driven directed lines, Phys. Rev. B 53 (1996) 3520-42.

[28] L. Balents, J.P. Bouchaud and M. Mézard, The large scale energy landscape of randomly pinned objects, J. Phys. I (France) 6 (1996) 1007-20.

[29] M. Kardar, Nonequilibrium dynamics of interfaces and lines, Phys. Rep. 301 (1998) 85-112.

[30] H. Leschhorn, T. Nattermann, S. Stepanow and L.-H. Tang, Driven interface depinning in a disordered medium, Annalen der Physik 6 (1997) 1-34.

[31] H. Bucheli, O.S. Wagner, V.B. Geshkenbein, A.I. Larkin and G. Blatter, $(4+N)$-dimensional elastic manifolds in random media: a renormalization-group analysis, Phys. Rev. B 57 (1998) 7642-52.

[32] D.S. Fisher, Collective transport in random media: from superconductors to earthquakes, Phys. Rep. 301 (1998) 113-150.

[33] Yusuf Dincer, Zur Universalität der Struktur elastischer Mannigfaltigkeiten in Unordnung, Master's thesis, Universität Köln, 81999.

[34] S. Scheidl, Private communication about 2-loop calculations for the random manifold problem. 20002004.

[35] S. Scheidl and Y. Dincer, Interface fluctuations in disordered systems: Universality and non-gaussian statistics, cond-mat/0006048 (2000). 
[36] T. Nattermann and S. Scheidl, Vortex-glass phases in type-ii superconductors, Advances in Physics 49 (2000) 607-704.

[37] DA. Gorokhov, DS. Fisher and G. Blatter, Quantum collective creep: a quasiclassical Langevin equation approach, Phys. Rev. B 66 (2002) 214203.

[38] J. M. Schwarz and Daniel S. Fisher, Depinning with dynamic stress overshoots: A hybrid of critical and pseudohysteretic behavior, cond-mat/0204623 (2002).

[39] G. Gruner, The dynamics of charge-density waves, Rev. of Mod. Phys. 60 (1988) 1129-81.

[40] S. Lemerle, J. Ferré, C. Chappert, V. Mathet, T. Giamarchi and P. Le Doussal, Domain wall creep in an Ising ultrathin magnetic film, Phys. Rev. Lett. 80 (1998) 849.

[41] A. Prevost, E. Rolley and C. Guthmann, Thermally activated motion of the contact line of a liquid ${ }^{4}$ He meniscus on a cesium substrate, Phys. Rev. Lett. 83 (1999) 348-51.

[42] A. Prevost, E. Rolley and C. Guthmann, Dynamics of a helium-4 meniscus on a strongly disordered cesium substrate, Phys. Rev. B 65 (2002) 064517/1-8.

[43] S. Moulinet, C. Guthmann and E. Rolley, Roughness and dynamics of a contact line of a viscous fluid on a disordered substrate, Eur. Phys. J. A 8 (2002) 437-43.

[44] T. Giamarchi and P. Le Doussal, Statics and dynamics of disordered elastic systems, in A.P. Young, editor, Spin glasses and random fields, World Scientific, Singapore, 1997, cond-mat/9705096.

[45] M. Kardar, G. Parisi and Y.-C. Zhang, Dynamic scaling of growing interfaces, Phys. Rev. Lett. 56 (1986) 889-892.

[46] M. Lassig and H. Kinzelbach, Upper critical dimension of the Kardar-Parisi-Zhang equation, Phys. Rev. Lett. 78 (1997) 903-6.

[47] E. Marinari, A. Pagnani and G. Parisi, Critical exponents of the KPZ equation via multi-surface coding numerical simulations, J. Phys. A 33 (2000) 8181-92.

[48] M. Lässig, On the renormalization of the Kardar-Parisi-Zhang equation, Nucl. Phys. B 448 (1995) 559-574.

[49] K.J. Wiese, On the perturbation expansion of the KPZ-equation, J. Stat. Phys. 93 (1998) 143-154, cond-mat/9802068.

[50] K.J. Wiese, The functional renormalization group treatment of disordered systems: a review, Ann. Henri Poincaré 4 (2003) 473-496, cond-mat/0302322.

[51] L. Balents and P. Le Doussal, Field theory of statics and dynamics of glasses: rare events and barrier distributions, cond-mat/0205358 (2002).

[52] L. Balents and P. Le Doussal, Broad relaxation spectrum and the field theory of glassy dynamics for pinned elastic systems, cond-mat/0312338 (2003).

[53] P. Le Doussal and K.J. Wiese, 2-loop functional renormalization group treatment of random field models, in preparation. 
[54] K.J. Wiese and P. Le Doussal, 3-loop FRG study of pinned manifolds, in preparation.

[55] K.B. Efetov and A.I. Larkin, Sov. Phys. JETP 45 (1977) 1236.

[56] M. Mézard, G. Parisi and M.A. Virasoro, Spin Glas Theory and Beyond, World Scientific, Singapore, 1987.

[57] A.A. Middleton, Energetics and geometry of excitations in random systems, Phys. Rev. B 63 (2001) 060202.

[58] YY. Goldschmidt, The 1/d expansion for the quantum mechanical n-body problem. application for directed polymers in a random medium, Nucl. Phys. B 393 (1993) 507-22.

[59] C. De Dominicis, I. Kondor and T. Temesvari, Dyson's equations for the Ising spin-glass, J. Phys. I (France) 4 (1994) 1287-308.

[60] D.M. Carlucci, C. De Dominicis and T. Temesvari, Stability of the Mezard-Parisi solution for random manifolds, J. Phys. I (France) 6 (1996) 1031-41.

[61] C. De Dominicis, Beyond the Sherrington-Kirkpatrick Model, in A.P. Young, editor, Spin glasses and random fields, World Scientific, Singapore, 1997.

[62] E. Brezin and C. De Dominicis, New phenomena in the random field Ising model, Europhys. Lett. 44 (1998) 13-19.

[63] E. Brezin and C. De Dominicis, Interactions of several replicas in the random field ising model, Eur. Phys. J. B 19 (2001) 467-71.

[64] E. Brézin and C. De Dominicis, Twist free energy in a spin glass, cond-mat/0201066 (2002).

[65] E. Brézin and C. De Dominicis, Twist free energy, cond-mat/0201069 (2002).

[66] C. De Dominicis and E. Bŕezin, On a dynamical-like replica-symmetry-breaking scheme for the spin glass, cond-mat/0402629 (2004).

[67] W. Zimmermann, Convergence of Bogoliubov's method of renormalization in monmentum space, Commun. Math. Phys. 15 (1969) 208-234.

[68] K. Hepp, Proof of the Bogoliubov-Parasiuk theorem on renormalization, Comm. Math. Phys. 2 (1966) 301-326.

[69] N.N. Bogoliubov and O.S. Parasiuk, Über die Multiplikation der Kausalfunktionen in der Quantentheorie der Felder, Acta Math. 97 (1957) 227.

[70] M.C. Bergere and Y.-M.P. Lam, Bogoliubov-Parasiuk theorem in the $\alpha$-parametric representation, J. Math. Phys. 17 (1976) 1546-1557. 